\title{
Utilizing IQ Mixers for Phase Noise Cancellation In Full-Duplex Architectures
}

\author{
by \\ Michael Feuerherm \\ A thesis submitted to the Faculty of Graduate and Postdoctoral Affairs \\ IN PARTIAL FULFILLMENT OF THE REQUIREMENTS FOR THE DEGREE OF
}

Master of Applied Science

in

Electrical and Computer Engineering

Carleton University

Ottawa, Ontario

(C) 2019

Michael Feuerherm 


\begin{abstract}
Full-duplex transceivers are typically used in both RADAR and RFID applications, as this architecture transmits and receives at the same time, a problem referred to as self-interference occurs. The transmitted signal leaks into the receiver and the phase noise of the leaked signal interferes with the returned receive signal. This thesis proposes, analyzes and measures a novel method of self-interference cancellation in fullduplex transceivers through the use of an IQ mixer and system design constraints. The original Local Oscillator of the transmitter is coupled and used for down conversion, if the system can be assumed to be linear, and time-invariant the phase noise of the self-interference can be estimated, and significantly reduced. This method was shown to achieve cancellation of up to $32.6 \mathrm{~dB}$, however theoretically the limits of this method are constrained by the sensitivity of the measurement and matching systems used.
\end{abstract}




\section{Dedication}

I would like to dedicate this thesis to Peaches for helping me get through a very hard time in my life. 


\section{Acknowledgements}

I would like to express my gratitude to a number of people, firstly my thesis supervisors: John Rogers, and Jim Wight for guiding me throughout my degree. Thank you for all of the opportunities and expertise you have shared with me.

I would also like to thank the people at Teslonix Inc., Vytas Kezys and Bill Bereza for their assistance with the research and implementation of my thesis as well as Daniel Azzoni Gravel for his comic relief.

In addition, I would like to thank the staff of the Department of Electronics at Carleton University, without their help this thesis would not have been possible.

I would like to thank all of my family and friends for their continued love and support. Specifically I would like to thank Spencer Whyte for his patience when answering all of my questions and taking time out of his busy life to help me.

I would like to thank Mieka Shade for all of her love and support over my academic career.

Lastly, I would like to thank Phil Beingessner for helping me discover my love for engineering and science years ago. 


\section{Contents}

1 Introduction 1

1.1 Motivation . . . . . . . . . . . . . . . . . . . 1

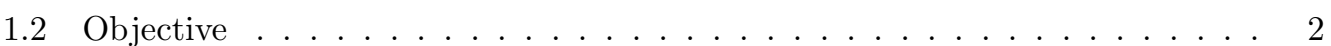

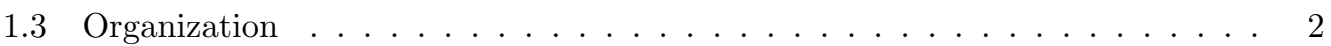

2 Background Information $\quad 4$

2.1 Phase Noise Sources . . . . . . . . . . . . . . . . . . . . . . . . 4

$2.1 .1 \quad$ Flicker Noise . . . . . . . . . . . . . . . . . . . . 7

$2.1 .2 \quad$ Johnson Noise . . . . . . . . . . . . . . . . . . . . . . . . . 8

2.1 .3 Shot Noise . . . . . . . . . . . . . . . . . . . . 9

2.2 Phase Noise Measurement . . . . . . . . . . . . . . . . . . . . . . . 10

2.2 .1 Direct Spectrum Measurement . . . . . . . . . . . . . . 10

2.2.2 Two-Channel Cross-Correlation Measurement . . . . . . . . . . . . . . 11

2.2.3 Phase Detector Technique Using Reference Source Method . . . . . . 12

2.3 Linear Time-Invariant Systems and Signal Processing . . . . . . . . . . . . 15

2.3 .1 Time Invariant Systems . . . . . . . . . . . . . . . . . . . . 15

$2.3 .2 \quad$ Linear Systems . . . . . . . . . . . . . . . . . . . . . . . 16

2.3.3 Spectral Leakage and Windowing . . . . . . . . . . . . . . 17 
2.3.4 Decimation and Quantization Noise Reduction . . . . . . . . . . 20

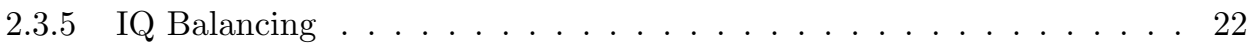

2.4 UHF RFID and Self-Interference . . . . . . . . . . . . . . . . 25

2.4.1 On-Off Shift Keying and Pulse Interval Encoding . . . . . . . . . . 25

2.4 .2 Self-Interference . . . . . . . . . . . . . . . 27

2.5 Current Solutions to Self-Interference . . . . . . . . . . . . . . . . 29

2.5.1 Code-domain Spread Spectrum Modulation . . . . . . . . . . . 29

2.5.2 Highly Integrated Tunable Duplexing Transceiver . . . . . . . . . . . . 30

2.5.3 Magnetic-Free N-path-Filter Non-Reciprocal Circulator and Base-band Self-Interference Cancellation . . . . . . . . . . . . . . . . 32

3 Mixer Phase Noise Cancellation System Analysis

3.1 Determining Initial Inputs . . . . . . . . . . . . . . . . . . . . 37

3.1.1 Input $\mathrm{a}(\mathrm{t}) \ldots \ldots \ldots \ldots \ldots$

3.1 .2 Input $b(t) \ldots \ldots \ldots \ldots$

3.1.3 Input $\mathrm{c}(\mathrm{t}) \ldots \ldots \ldots \ldots$. . . . . . . . . . . . . . . . . . . .

3.2 Mixing Signals and Baseband Power . . . . . . . . . . . . . . . 39

3.2.1 Output $\mathrm{I}(\mathrm{t}) \ldots \ldots \ldots \ldots$

3.2.2 Output $\mathrm{Q}(\mathrm{t}) \ldots \ldots \ldots \ldots \ldots$ 
3.2.3 Mixer Output and Phase Noise Cancellation . . . . . . . . . . . 41

4 Test System Implementation and Measurements

4.1 System Implementation . . . . . . . . . . . . . . . . . . . . . . . . 45

4.2 Component Characterization . . . . . . . . . . . . . . . . 47

4.2 .1 IQ Correction . . . . . . . . . . . . . . . . . . . . . 48

4.2 .2 IQ Mixer Gain Characterization . . . . . . . . . . . . . . . 51

4.2 .3 IQ Mixer Phase Characterization . . . . . . . . . . . . . 55

4.2.4 Synthesizer Phase Noise Characterization . . . . . . . . . . . . 66

4.3 Phase Noise Cancellation Measurements . . . . . . . . . . . . . . . . . . . 71

4.3.1 Phase Noise Cancellation of 90m SMA Cable System . . . . . . . . . . 74

4.3.2 Phase Noise Cancellation of 80m SMA Cable System . . . . . . . . . . 80

4.3.3 Phase Noise Cancellation of $70 \mathrm{~m}$ SMA Cable System . . . . . . . . . . 85

4.3.4 Phase Noise Cancellation of 60m SMA Cable System . . . . . . . . . . 90

4.3.5 Phase Noise Cancellation of 50m SMA Cable System . . . . . . . . . . 95

4.3.6 Phase Noise Cancellation of 40m SMA Cable System . . . . . . . . . . 100

4.3.7 Phase Noise Cancellation of 30m SMA Cable System . . . . . . . . . 105

4.3 .8 Cancellation Overview . . . . . . . . . . . . . . . . . . . . . 110

5 Conclusion and Future Work $\quad 124$ 
5.1 Contributions . . . . . . . . . . . . . . . . . . . . . . . . 124

5.2 Conclusion . . . . . . . . . . . . . . . . . . . . . . . . . . . 124

5.3 Future Work . . . . . . . . . . . . . . . . . . . . . . . 125 


\section{List of Figures}

1 Jitter Context . . . . . . . . . . . . . . . . . . . . 4

2 Typical Phase Noise Profile . . . . . . . . . . . . . . . . 6

3 Thermal Noise Model ........................ 8

4 Phase Noise Direct Spectrum Measurement Schematic . . . . . . . . . . . . . 11

5 Phase Noise Two-Channel Cross-Correlation Measurement Schematic . . . . . 11

6 Phase Detector Diagram . . . . . . . . . . . . . . . . . . 12

7 Phase Detector: Reference Source Measurement Schematic _ . . . . . . . . . 13

8 Phase Detector: Modified Reference Source Measurement Schematic . . . . . 14

9 Applying Window to Signal . . . . . . . . . . . . . . . . . . 18

10 Original Spectrum of Arbitrary Signal . . . . . . . . . . . . . . . . . 18

11 Windowed Spectrum of Arbitrary Signal . . . . . . . . . . . . . . . . . . 19

12 Spectrum of Quantization Noise for Typical ADC . . . . . . . . . . . . . 21

13 Spectrum of Quantization Noise for Oversampled Decimated ADC . . . . . . 21

14 Simplified RFID Link . . . . . . . . . . . . . . . . . . 26

15 On-Off Keyed Signal . . . . . . . . . . . . . . . . . . . 27

16 CSSM and On-Chip Duplexer System Level Diagram . . . . . . . . . . . . . . 29

17 CSSM full-duplex transceiver front-end architecture . . . . . . . . . . . 30 
18 System Diagram of Transceiver . . . . . . . . . . . . . . . . . . . . 31

19 Circuit Diagram of Duplexing LNA . . . . . . . . . . . . . . . . . 32

20 N-path-Filter Non-Reciprocal Circulator System Diagram . . . . . . . . . . . 33

21 N-path-Filter Non-Reciprocal Circulator System Diagram . . . . . . . . . . . 34

22 Mixer Phase Noise Cancellation Circuit Diagram . . . . . . . . . . . . . 36

23 System Diagram _ . . . . . . . . . . . . . . . . . . 37

24 IQ Mixer Schematic . . . . . . . . . . . . . . . . . . . 46

$25 \quad$ RF Input Schematic . . . . . . . . . . . . . . . . . . . . . . . 47

26 Local Oscillator Input Schematic . . . . . . . . . . . . . . . . . . . . . 47

27 Time Domain $1 \mathrm{MHz}$ Signal Before Correction . . . . . . . . . . . . . . . . 48

28 Frequency Domain 1 MHz Signal Spectrum Before Correction . . . . . . . . . 49

29 Time Domain $1 \mathrm{MHz}$ Signal After Correction . . . . . . . . . . . . . . . 50

30 Frequency Domain $1 \mathrm{MHz}$ Signal Spectrum After Correction . . . . . . . . . 50

31 IQ Mixer Gain Characterization Schematic . . . . . . . . . . . . . . . 51

32 Mixer LO Input Spectrum . . . . . . . . . . . . . . . . . . . . . . . . 52

$3390^{\circ}$ Phase-Shifted Mixer LO Input Spectrum . . . . . . . . . . . . . . . . 52

34 Output Power versus Input Power Of IQ Mixer . . . . . . . . . . . . . . 53

35 Beginning of Compression of IQ Mixer Output . . . . . . . . . . . . . 55 
36 IQ Mixer Phase Response Characterization Schematic . . . . . . . . . . . . 56

37 Input Signal for $11 \mathrm{~cm}$ SMA Cable With $16 \mathrm{~dB}$ Attenuator . . . . . . . . . 57

38 IQ Data Using $11 \mathrm{~cm}$ SMA Cable . . . . . . . . . . . . . . . . . . 57

39 Mean of IQ Data Using $11 \mathrm{~cm}$ SMA Cable . . . . . . . . . . . . . . . . 58

40 IQ Data Using $15 \mathrm{~cm}$ SMA Cable . . . . . . . . . . . . . . . . 58

41 Mean of IQ Data Using $15 \mathrm{~cm}$ SMA Cable . . . . . . . . . . . . . . . . . 59

42 IQ Data Using $30 \mathrm{~cm}$ SMA Cable . . . . . . . . . . . . . . . . . . 59

43 Mean of IQ Data Using $30 \mathrm{~cm}$ SMA Cable . . . . . . . . . . . . . . . . 60

44 IQ Data Using $61 \mathrm{~cm}$ SMA Cable . . . . . . . . . . . . . . . . . 60

45 Mean of IQ Data Using $30 \mathrm{~cm}$ SMA Cable . . . . . . . . . . . . . . 61

46 Corrected IQ Data Using $11 \mathrm{~cm}$ SMA Cable . . . . . . . . . . . . . . . . 62

47 Corrected Mean of IQ Data Using $11 \mathrm{~cm}$ SMA Cable . . . . . . . . . . . . 62

48 Corrected IQ Data Using $15 \mathrm{~cm}$ SMA Cable . . . . . . . . . . . . . 63

49 Corrected Mean of IQ Data Using $15 \mathrm{~cm}$ SMA Cable . . . . . . . . . . . . 63

50 Corrected IQ Data Using $30 \mathrm{~cm}$ SMA Cable . . . . . . . . . . . . . . . . 64

51 Corrected Mean of IQ Data Using 30cm SMA Cable . . . . . . . . . . . . . . 64

52 Corrected IQ Data Using $61 \mathrm{~cm}$ SMA Cable . . . . . . . . . . . . . 65

53 Corrected Mean of IQ Data Using 30cm SMA Cable . . . . . . . . . . . . . 65 
54 Synthesizer Phase Noise Characterization Schematic . . . . . . . . . . . . 67

55 RF Input Terminated Schematic . . . . . . . . . . . . . . . . 67

56 Measured Noise Floor With RF Input Terminated . . . . . . . . . . . . . 68

57 Measured Noise Floor With RF Input Terminated From -100 kHz to 100 kHz 68

58 Phase Noise Spectral Density of Two Synthesizers _ . . . . . . . . . . . 69

59 Phase Noise Spectral Density of Two Synthesizers From -100 kHz to 100 kHz 70

60 Synthesizer Phase Noise Cancellation Schematic _ . . . . . . . . . . . 71

61 System Under Test Schematic . . . . . . . . . . . . . . . . . . . . 72

62 Splitter Output Power Schematic . . . . . . . . . . . . . . . . . . 72

63 LO Split Signal Spectral Power . . . . . . . . . . . . . . . . . . 73

$64 \quad$ RF Input Power Schematic . . . . . . . . . . . . . . . . . . . . 75

65 RF Input Power of $90 \mathrm{~m}$ System . . . . . . . . . . . . . . 76

66 Phase Noise Spectral Density of $90 \mathrm{~m}$ System _ . . . . . . . . . . 76

67 Phase Noise Spectral Density of $90 \mathrm{~m}$ System From $-100 \mathrm{kHz}$ to $100 \mathrm{kHz}$. . . 77

68 RF Input Power of $80 \mathrm{~m}$ System . . . . . . . . . . . . . . . . . . 81

69 Phase Noise Spectral Density of $80 \mathrm{~m}$ System . . . . . . . . . . . . . . 82

70 Phase Noise Spectral Density of $80 \mathrm{~m}$ System From $-100 \mathrm{kHz}$ to $100 \mathrm{kHz}$. . . 82

$71 \quad$ RF Input Power of $70 \mathrm{~m}$ System . . . . . . . . . . . . . . 86 
72 Phase Noise Spectral Density of $70 \mathrm{~m}$ System $\ldots \ldots$. . . . . . . . . . 87

73 Phase Noise Spectral Density of $70 \mathrm{~m}$ System From $-100 \mathrm{kHz}$ to $100 \mathrm{kHz}$. . . 87

$74 \quad$ RF Input Power of $60 \mathrm{~m}$ System . . . . . . . . . . . . . . . . . . . 91

75 Phase Noise Spectral Density of $60 \mathrm{~m}$ System _ . . . . . . . . . . . . . 92

76 Phase Noise Spectral Density of $60 \mathrm{~m}$ System From $-100 \mathrm{kHz}$ to $100 \mathrm{kHz}$. . . 92

77 RF Input Power of $50 \mathrm{~m}$ System . . . . . . . . . . . . . . . 96

78 Phase Noise Spectral Density of $50 \mathrm{~m}$ System $\ldots \ldots \ldots$. . . . . . . . . 97

79 Phase Noise Spectral Density of $50 \mathrm{~m}$ System From $-100 \mathrm{kHz}$ to $100 \mathrm{kHz}$. . . 97

80 RF Input Power of $40 \mathrm{~m}$ System . . . . . . . . . . . . . . . . . 101

81 Phase Noise Spectral Density of $40 \mathrm{~m}$ System . . . . . . . . . . . . . . . 102

82 Phase Noise Spectral Density of 40m System From -100 kHz to $100 \mathrm{kHz}$. . . 102

83 RF Input Power of 30m System . . . . . . . . . . . . . . . . . . 106

84 Phase Noise Spectral Density of 30m System _ . . . . . . . . . . . . . 107

85 Phase Noise Spectral Density of 30m System From -100 kHz to $100 \mathrm{kHz}$. . . 107

86 Change In Phase Noise Spectral Density Relative to Carrier at $20 \mathrm{kHz}$ Offset 111

87 Change In Phase Noise Spectral Density Relative to Carrier at $50 \mathrm{kHz}$ Offset 112

88 Change In Phase Noise Spectral Density Relative to Carrier at $100 \mathrm{kHz}$ Offset 113

89 Change In Phase Noise Spectral Density Relative to Carrier at $300 \mathrm{kHz}$ Offset 114 
90 Change In Phase Noise Spectral Density Relative to Carrier at $1000 \mathrm{kHz}$ Offset116

91 Change In Phase Noise Spectral Density Relative to Carrier at $20 \mathrm{kHz}$ Offset 118

92 Change In Phase Noise Spectral Density Relative to Carrier at 50 kHz Offset 119

93 Change In Phase Noise Spectral Density Relative to Carrier at $100 \mathrm{kHz}$ Offset 120

94 Change In Phase Noise Spectral Density Relative to Carrier at $300 \mathrm{kHz}$ Offset 121

95 Change In Phase Noise Spectral Density Relative to Carrier at $1000 \mathrm{kHz}$ Offset122 


\section{List of Tables}

1 Gain Measurement Results of IQ Mixer . . . . . . . . . . . . . . . . . . . 54

2 Electrical Lengths of Selected SMA Cables . . . . . . . . . . . . . . . . . 56

3 Phase Response Measurement Results of IQ Mixer . . . . . . . . . . . . . . . 61

4 Corrected Phase Response Measurement Results of IQ Mixer . . . . . . . . . 66

$5 \quad$ Noise Floor Spectral Density With RF Input Terminated . . . . . . . . . . . 69

$6 \quad$ Measured Phase Noise Spectral Density . . . . . . . . . . . . . . . . . 70

$7 \quad$ Phase Noise Spectral Density of TPI Synthesizer . . . . . . . . . . . . 71

8 System Response Measurements Of 90m SMA Cable . . . . . . . . . . . . . . 74

9 Phase Noise Spectral Density of $90 \mathrm{~m}$ system . . . . . . . . . . . . . . . 75

$1090 \mathrm{~m}$ Cable Noise Floor Spectral Density With RF Input Terminated . . . . . 77

11 Phase Noise Of $90 \mathrm{~m}$ SMA Cable $\ldots \ldots \ldots \ldots \ldots$

12 Phase Noise Spectral Density of $90 \mathrm{~m}$ system . . . . . . . . . . . . . . 78

13 Cancellation Measurement for $90 \mathrm{~m}$ Of Cable . . . . . . . . . . . . . . 79

14 System Response Measurements Of 80m SMA Cable . . . . . . . . . . . . 80

15 Phase Noise Spectral Density of $80 \mathrm{~m}$ system . . . . . . . . . . . . . . . 81

$1680 \mathrm{~m}$ Cable Noise Floor Spectral Density With RF Input Terminated . . . . . 83

17 Phase Noise Of $80 \mathrm{~m}$ SMA Cable $\ldots \ldots \ldots \ldots$ 
18 Phase Noise Spectral Density of $80 \mathrm{~m}$ system . . . . . . . . . . . . . . . . . 84

19 Cancellation Measurement for $80 \mathrm{~m}$ Of Cable . . . . . . . . . . . . . . . 84

20 System Response Measurements Of $70 \mathrm{~m}$ SMA Cable . . . . . . . . . . . . . 85

21 Phase Noise Spectral Density of $70 \mathrm{~m}$ system . . . . . . . . . . . . . . . 86

$2270 \mathrm{~m}$ Cable Noise Floor Spectral Density With RF Input Terminated . . . . . 88

23 Phase Noise Of $70 \mathrm{~m}$ SMA Cable $\ldots \ldots \ldots \ldots$. . . . . . . . . 88

24 Phase Noise Spectral Density of $70 \mathrm{~m}$ system . . . . . . . . . . . . . . . . . 89

25 Cancellation Measurement for $70 \mathrm{~m}$ Of Cable . . . . . . . . . . . . . . 89

26 System Response Measurements Of 60m SMA Cable . . . . . . . . . . . . . . 90

27 Phase Noise Spectral Density of $60 \mathrm{~m}$ system . . . . . . . . . . . . . . . . . 91

28 60m Cable Noise Floor Spectral Density With RF Input Terminated . . . . . 93

29 Phase Noise Of $60 \mathrm{~m}$ SMA Cable . . . . . . . . . . . . . . . . . . . . . 93

30 Phase Noise Spectral Density of $60 \mathrm{~m}$ system . . . . . . . . . . . . . . . . . 94

31 Cancellation Measurement for $60 \mathrm{~m}$ Of Cable . . . . . . . . . . . . . 94

32 System Response Measurements Of 50m SMA Cable . . . . . . . . . . . . . . 95

33 Phase Noise Spectral Density of $50 \mathrm{~m}$ system . . . . . . . . . . . . . . . . 96

$3450 \mathrm{~m}$ Cable Noise Floor Spectral Density With RF Input Terminated . . . . . 98

35 Phase Noise Of $50 \mathrm{~m}$ SMA Cable . . . . . . . . . . . . . . . . . . . 98 
36 Phase Noise Spectral Density of $50 \mathrm{~m}$ system . . . . . . . . . . . . . . . . . 99

37 Cancellation Measurement for 50m Of Cable . . . . . . . . . . . . . . 99

38 System Response Measurements Of 40m SMA Cable . . . . . . . . . . . . . . 100

39 Phase Noise Spectral Density of $40 \mathrm{~m}$ system . . . . . . . . . . . . . . . . . . 101

$4040 \mathrm{~m}$ Cable Noise Floor Spectral Density With RF Input Terminated . . . . . 103

41 Phase Noise Of $40 \mathrm{~m}$ SMA Cable . . . . . . . . . . . . . . . . . . 103

42 Phase Noise Spectral Density of $40 \mathrm{~m}$ system . . . . . . . . . . . . . . . . . . . 104

43 Cancellation Measurement for 40m Of Cable . . . . . . . . . . . . . . . . . 104

44 System Response Measurements Of 30m SMA Cable . . . . . . . . . . . . . 105

45 Phase Noise Spectral Density of 30m system . . . . . . . . . . . . . . . . 106

$46 \quad 30 \mathrm{~m}$ Cable Noise Floor Spectral Density With RF Input Terminated . . . . . 108

47 Phase Noise Of $30 \mathrm{~m}$ SMA Cable . . . . . . . . . . . . . . . . . . . 108

48 Phase Noise Spectral Density of 30m system . . . . . . . . . . . . . . . . . 109

49 Cancellation Measurement for 30m Of Cable . . . . . . . . . . . . . . . 109

50 Calculated and Measured Change in Phase Noise at $20 \mathrm{KHz}$ Offset . . . . . . 110

51 Calculated and Measured Change in Phase Noise at $50 \mathrm{KHz}$ Offset . . . . . . 112

52 Calculated and Measured Change in Phase Noise at $100 \mathrm{KHz}$ Offset . . . . . 113

53 Calculated and Measured Change in Phase Noise at $300 \mathrm{KHz}$ Offset . . . . . 114 
54 Calculated and Measured Change in Phase Noise at $1000 \mathrm{KHz}$ Offset . . . . . 115

55 Expected and Measured Output Power of Systems Under Test . . . . . . . 117

56 Calculated and Modified Measured Change in Phase Noise at $20 \mathrm{KHz}$ Offset . 118

57 Calculated and Modified Measured Change in Phase Noise at $50 \mathrm{KHz}$ Offset . 119

58 Calculated and Modified Measured Change in Phase Noise at $100 \mathrm{KHz}$ Offset 120

59 Calculated and Modified Measured Change in Phase Noise at $300 \mathrm{KHz}$ Offset 121

60 Calculated and Modified Measured Change in Phase Noise at $1000 \mathrm{KHz}$ Offset 122 


\section{Introduction}

This thesis will discuss the theory and implementation of a mixer based method of phase noise cancellation. In systems which utilize full-duplex transceiver architectures, such as Radio Frequency Identification (RFID) or Radio Detection and Ranging (RADAR), an oscillator is used to mix the back-scattered received signal directly down to DC, this causes the modulated data from the received signal to mix to baseband. An undesired secondary effect is that a leaked transmit signal, due to non-perfect isolation, or reflection from the antenna due to impedance mismatch for example, is also down converted to DC. The phase noise of the leaked signal then occurs at baseband, and interferes with the desired modulated data of the received signal - this phenomena is referred to as self-interference.

A system using a time delay element to provide phase noise cancellation utilizing a mixer has been previously analyzed, this thesis will provide a novel method for cancellation, which will function for a larger set of applications [1]. This thesis will derive the formula seen in equation 1, which utilizes the response of a given linear time-invariant system at the center frequency and some frequency offset of interest, in order to predict the amplitude of phase noise cancellation the system will achieve using this method.

$$
C_{P N}=\left|e^{-i \theta_{0}}-\frac{s_{21-}}{s_{210}} e^{-i \theta_{-}}\right|^{2}+\left|e^{-i \theta_{0}}-\frac{s_{21+}}{s_{210}} e^{-i \theta_{+}}\right|^{2}
$$

Following this derivation measurements will be shown in order to prove the application and effectiveness of the equation.

\section{$1.1 \quad$ Motivation}

As society continues to shift towards a significantly more automated lifestyle, technology must continue to improve to accommodate the increased demand for automation. A 
widespread tool used for automation is the use of RFID tags and readers which are used to track, and monitor various objects [2] [3]. These RFID tags generally use a continuous wave from a RFID reader ( $\mathrm{RF}$ transceiver) in order to power themselves and transmit modulated data back to the reader through the use of back-scatter [4].

Due to the RFID tags requiring a continuous wave to power them, the RFID reader must have a full duplex design in order to receive the returned signal from the RFID tag while continuing to transmit the continuous wave. Unfortunately as the continuous wave and back-scattered signal occur simultaneously the original transmitted signal is leaked into the receiver. The phase noise of this original signal occurs at the same frequency of the modulated data of the tag, therefore the phase noise can degrade the ability of the receiver to read the wanted modulated back-scatter signal [4]. In order to maximize the ability to receive smaller power signals within full duplex architectures this phase noise should be attempted to be attenuated to the point where it negligibly affects the receiver sensitivity.

\subsection{Objective}

The objective of this thesis is to take an input oscillator signal with an associated phase noise process and attempt to significantly reduce the phase noise amplitude of the self-interference signal. Therefore this thesis will analyze, implement, and measure the results of a system representative of the desired system in order to verify its functionality.

\subsection{Organization}

This thesis will be structured into five separate chapters. Chapter 1 introduces the topic of the thesis, the motivation, and objective of the thesis. Chapter 2 will give background information concerning UHF RFID, sources of phase noise, phase noise measurement techniques, properties of Time-invariant linear systems, describe the various methods of signal processing that were implemented, as well as describe current solutions to self-interference 
of UHF RFID systems. Chapter 3 will discuss the theory and functionality behind the proposed system and provide an equation to approximate the resulting phase noise cancellation. Chapter 4 will present and discuss measurements and results for the implemented system. Lastly Chapter 5 will summarize the major results of the thesis, discuss the contributions of the thesis, and recommend future work. 


\section{Background Information}

Chapter 2 will discuss the various topics relevant to the examined mixer phase noise can-

cellation system. It will describe RFID, as well as its uses and limitations. It will also discuss the the various sources of phase noise, its relationship to jitter, their implications as well as methods of phase noise measurement. In addition it will discuss current solutions to self-interference. Lastly this chapter will discuss linear time-invariant systems, as well as the signal processing techniques used for measurement.

\subsection{Phase Noise Sources}

This section will discuss the various sources and implications of phase noise and jitter within oscillators. Phase noise and jitter refer to the same phenomena, where jitter is the timedomain representation and phase noise is the frequency domain representation of a change of a signal period [5]. An example signal showing the context of this change in signal period of a time varying signal can be seen below in Figure 1.

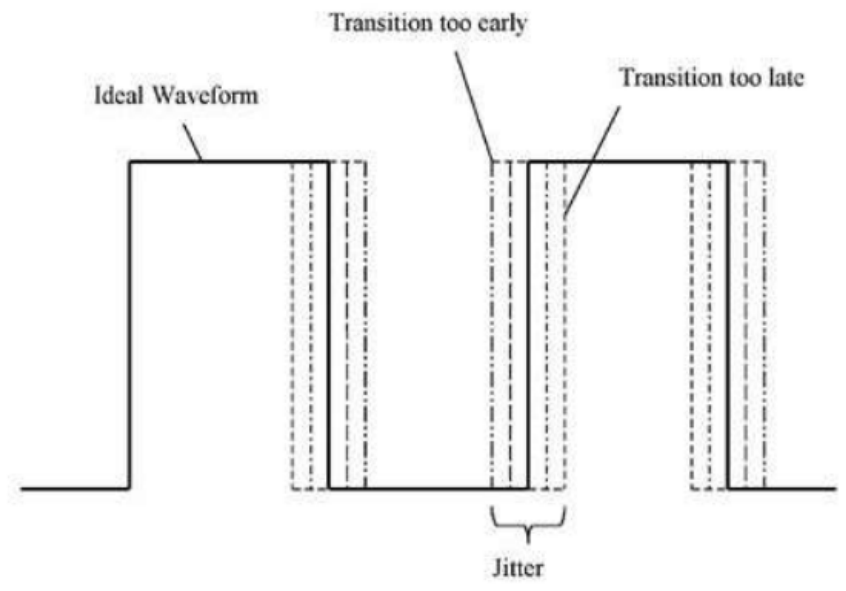

Figure 1: Jitter Context [5] 
As previously mentioned jitter and phase noise are different representations of the same phenomena, therefore there is clearly a relationship between phase noise and jitter. The jitter, in units of seconds, caused by a given phase noise can be calculated using equation 2 , where $\omega_{c}$ is the oscillator frequency, and $S_{\phi}(f)$ is the spectral density of the phase noise of interest [6].

$$
\operatorname{Jitter}_{R M S}=\frac{\sqrt{2 \cdot 10^{\frac{\int S_{\phi}(f)}{10}}}}{\omega_{c}}
$$

For the purposes of this thesis all calculations, measurements, and comparisons will be done using phase noise, as such it is important to properly define it. A typical phase noise profile of an oscillator can be seen below in Figure 2. This profile consists of a number of regions where a specific type of phase noise dominates. These regions are proportional to $\frac{1}{f^{x}}$ where $\mathrm{x}$ is a real integer greater than zero, for the purposes of this thesis the focus will typically be on the $\frac{1}{f^{2}}$ region as it is generally the main contributor to phase noise of an oscillator at our frequency of interest [7]. 


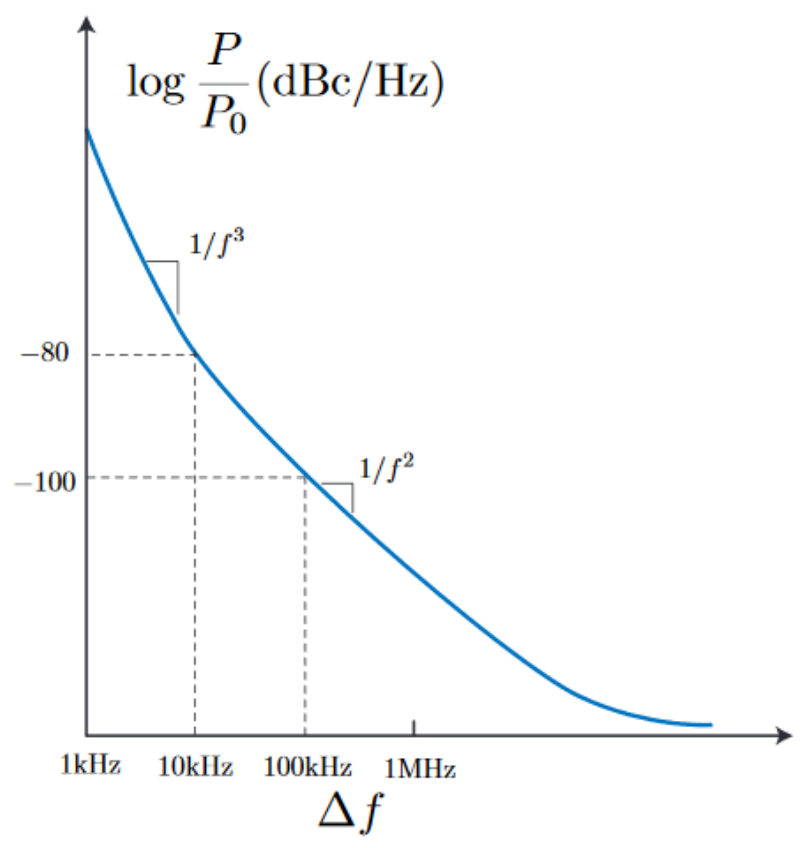

Figure 2: Typical Phase Noise Profile [8]

This phase noise can be represented mathematically using a variable defined as a phase noise process, represented as $\phi(\mathrm{t})$ is used within the signal [9]. The implementation of this phase noise process can be seen in equation 3 , which is a time domain equation of a complex sinusoidal signal.

$$
y(t)=\mathrm{e}^{\mathrm{i}\left(\omega_{c} t+\varphi(t)\right)}
$$

Noise in the frequency band on interest can be created through a number of sources such as Flicker Noise, Johnson Noise, Shot Noise. The following sections will describe the cause of these various noise sources and their relevance. In order to mathematically estimate the phase noise at a given frequency Leeson's Formula [10], seen in equation 4 which is an empirical model, is typically used. 


$$
y(t)=\left(\frac{\left|H_{1}\right| \omega_{0}}{2 Q \Delta \omega}\right)^{2} \frac{F k T}{2 P_{s}}
$$

Where $H_{1}$ is a constant determined by circuit characteristics, $\mathrm{Q}$ is the quality factor, $\omega_{0}$ is the output frequency, $\Delta \omega$ is the offset from the output frequency, $F$ is the excess noise factor for noise injected by sources outside of tank losses, $\mathrm{k}$ is the Boltzmann's constant, $\mathrm{T}$ is the temperature in Kelvin, and $P_{s}$ is the signal power of the carrier. However this equation assumes that the flicker noise is insignificant, therefore it can be corrected by adding a flicker noise term as seen below in equation $5[7]$, where $\omega_{c}$ is the corner frequency for where the flicker and thermal noise are approximately equal.

$$
y(t)=\left(\frac{\left|H_{1}\right| \omega_{0}}{2 Q \Delta \omega}\right)^{2}\left(1+\frac{\omega_{c}}{\Delta \omega}\right) \frac{F k T}{2 P_{s}}
$$

\subsubsection{Flicker Noise}

Flicker noise, a type of pink noise, or most commonly referred to as $\frac{1}{f}$ noise dominates relatively close to the carrier oscillator tone and consists of a number of regions. As seen in Figure 2 close to the carrier signal it can be higher power than the noise floor created by white Gaussian noise and has a roll-off rate of approximately $20 \mathrm{~dB} /$ decade in the frequency offset range of interest, this roll-off rate increases very close to the carrier [8]. There are two theories of mechanisms that are thought to create this noise; these are carrier number fluctuation and mobility fluctuation [11], it is generally debated which mechanism acts as the main contributor, therefore there is also a unified model that accounts for both mechanisms [12]. However it has been determined that the mechanisms that create $\frac{1}{f}$ noise within field effect transistors are linear while the mechanisms within bipolar junction transistors are at least partially non-linear [13]. 


\subsubsection{Johnson Noise}

Johnson Noise, also known as thermal noise, is created due to thermal changes of charge carriers within a circuit [14]. Thermal changes to charge carriers create a small random current which has constant power spectral density up to approximately $6000 \mathrm{GHz}[7]$. A common noise source within circuits is due to resistances, the thermal noise created by a resistance can be modelled using a voltage source in series with an ideal resistor as seen in Figure 3 , where $v_{n}$ is the noise voltage, and $\mathrm{R}$ is the ideal resistor.

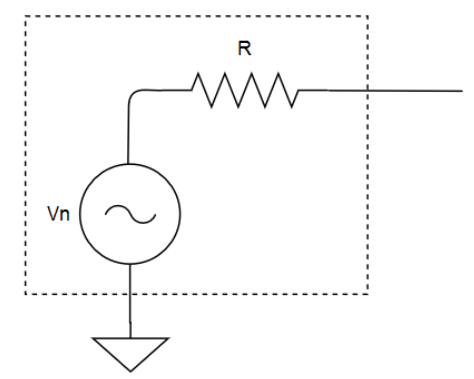

Figure 3: Thermal Noise Model [9]

The rms noise voltage can be calculated using equation 6 , where $\mathrm{k}$ is the Boltzmann's constant, $\mathrm{T}$ is the temperature in Kelvin, and $\mathrm{R}$ is the resistance value.

$$
v_{n}=\sqrt{4 k T R}
$$

This voltage can then be used to calculate the power produced by the resistance in a given bandwidth as seen in equation 7 .

$$
P=\frac{v_{n}^{2}}{R}=4 k T \Delta f
$$




\subsubsection{Shot Noise}

Shot Noise is typically only a small noise contributor within any relatively large signal circuits, however it can still have a measurable effect on small signals. It has a relatively simple mechanism for it's cause, shot noise is created due to the discrete nature of charge carriers within circuits [14]. As each charge carrier is a discrete particle they contribute a fixed amount to the total current, as such there can be small fluctuations in the instantaneous current, which over a long timescale creates noise on a given signal. The power of this noise can be calculated using the Schottky formula as seen below in equation 8 [15]. Where $I_{a v g}$ is the average current the noise appears on, $e_{0}$ is the charge of an electron, and $\Delta f$ is the bandwidth the noise is being calculated over.

$$
P_{\text {Shot }}=2 e_{0} I_{\text {avg }} \Delta f
$$




\subsection{Phase Noise Measurement}

This section will discuss some of the methods used to measure the phase noise of oscillators, and the selection of which method will be used to characterize phase noise in this thesis. There are a number of methods for measuring the phase noise of a given oscillator, these include but are not limited to: Direct Spectrum measurement, Phase Detector techniques, and the Two-channel cross-correlation technique [16]. Each of these measurements techniques has benefits and drawbacks, this section will discuss these as well as the implementations of these techniques.

\subsubsection{Direct Spectrum Measurement}

The first technique that will be discussed, and generally the most straightforward measurement method, is the direct spectrum technique. This technique uses an implementation seen in Figure 4, which takes the output of the device under test (DUT) and inputs it directly into a spectrum analyzer. As the implementation of this technique is very simplistic it offers a quick way to measure the phase noise of an oscillator, however it is limited due to the dynamic range of the spectrum analyzer being used because the carrier tone is present during

measurement. In order to utilize this method two criteria must be met: at the frequency of interest the single side-band (SSB) phase noise of the spectrum analyzer must be lower than the phase noise of the DUT, and if the spectrum analyzer cannot differentiate between amplitude noise and phase noise the amplitude noise must be significantly lower than the phase noise of interest [16]. 


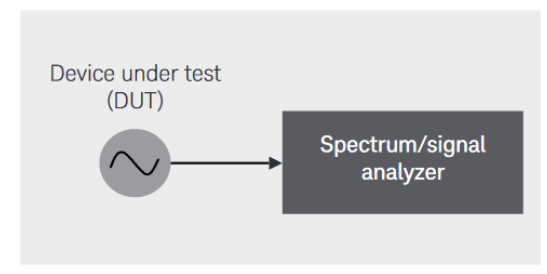

Figure 4: Phase Noise Direct Spectrum Measurement Schematic [16]

\subsubsection{Two-Channel Cross-Correlation Measurement}

The second technique for measuring phase noise is through the use of two duplicate PhaseLocked-Loop systems as seen below in, the outputs of which are used to perform cross correlation operations, the schematic for this method can be seen below in Figure 5 . As the DUT is passed into each the system the noise due to the DUT is coherent in each channel, while the added noise due to the channels are incoherent, therefore the cross correlation operation will not affect the DUT noise but the added channel noise will be reduced [16].

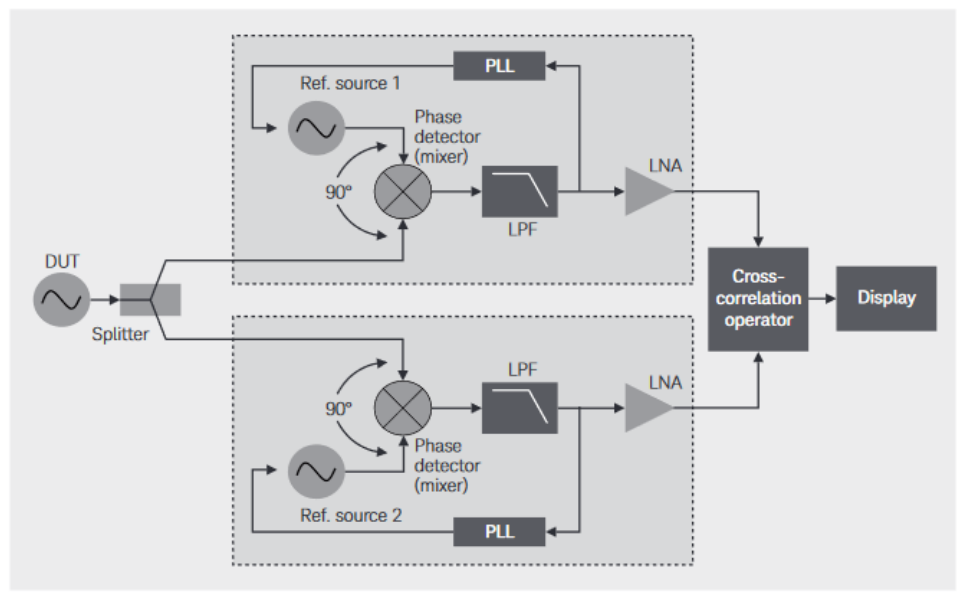

Figure 5: Phase Noise Two-Channel Cross-Correlation Measurement Schematic [16]

The theoretical measured noise can be calculated using equation 9 , where $N_{\text {meas }}$ is the total measure noise, $N_{D U T}$ is the noise due to the DUT, $N_{1}$ and $N_{2}$ are the noise of the 
channels 1 and 2 respectively, and $\mathrm{M}$ is the correlation coefficient [16]. Utilizing the crosscorrelation method allows for greater sensitivity during measurement without a requirement for high performance hardware, however due to cross-correlation processing increasing the correlation coefficient, and therefore sensitivity, it also increases the length of time required per measurement [16].

$$
N_{\text {meas }}=N_{D U T}+\frac{N_{1}+N_{2}}{\frac{M}{2}}
$$

\subsubsection{Phase Detector Technique Using Reference Source Method}

The last technique for measuring phase noise to be discussed is through the use of a phase detector. Utilizing a phase detector and two input signals, as seen in Figure 6, it is possible to separate the amplitude and phase noise of a given signal. If the phase difference between the two signals is $90^{\circ}$ the average of the resulting output voltage will be zero [16], while any fluctuation from $90^{\circ}$ will therefore cause a voltage fluctuation.

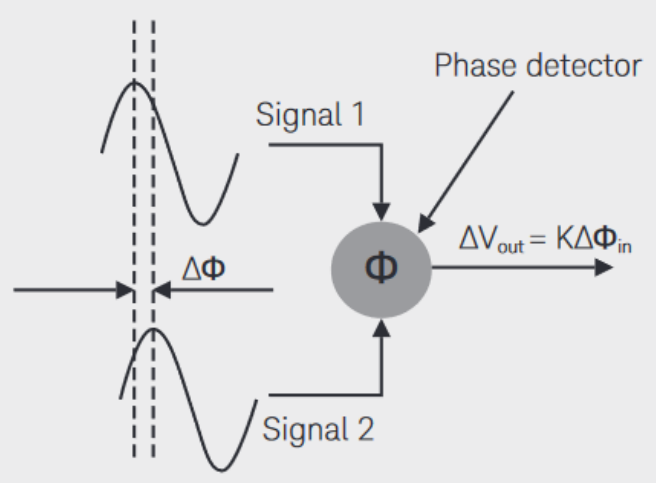

Figure 6: Phase Detector Diagram [16]

There are a number of methods for the implementation of a phase detector technique of measuring the phase noise, however this thesis will only go into discussion of the reference 
source/PLL method, and the modified version implemented. An example of a reference source implementation can be seen below in Figure 7. This method uses a mixer as a phase detector, taking one input from a known reference signal and the second from the DUT. Both signals are operated at the same frequency with the reference $90^{\circ}$ out of phase, therefore the mixing outputs occur at DC and twice the operating frequency. Once the signal is at baseband if the reference is well characterized it can be subtracted from the total phase noise to determine the phase noise of the DUT, if it is negligible the measured phase noise can be approximated to be the phase noise of the DUT.

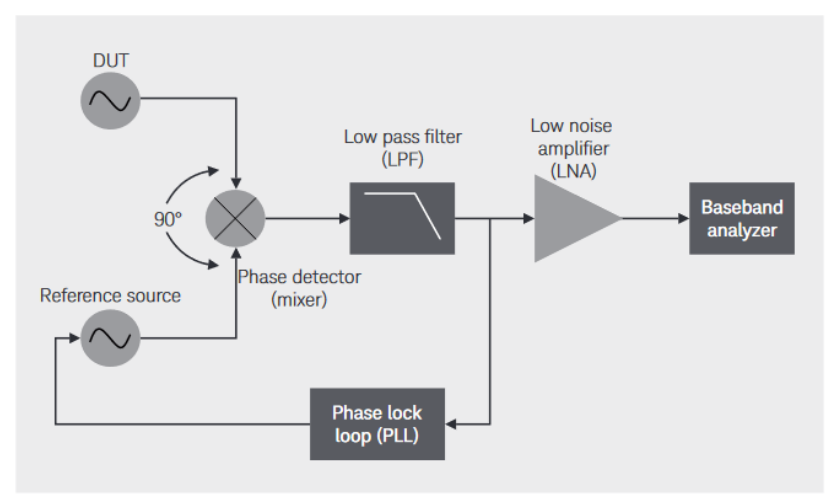

Figure 7: Phase Detector: Reference Source Measurement Schematic [16]

As mentioned previously the method used is a modified version of the reference source design. The known reference was replaced with a second synthesizer that utilized an identical Voltage Controlled Oscillator (VCO), and the same reference source was used in both synthesizers. The signal from the second synthesizer was put into Mixer 1 directly, however was passed through a $90^{\circ}$ phase shift before being fed into Mixer 2 in order to create the I and Q portions of the signal respectively, these I and Q signals were then used in order to construct the overall complex signal. Since the phase noise of the two VCOs will be approximately identical in power but will be non-coherent the resulting spectrum density will be approximately $3 \mathrm{~dB}$ higher than a single noise source [17]. Therefore the noise of a single source can be characterized by subtracting $3 \mathrm{~dB}$ from the measured spectral density, 
the schematic used for this method can be seen below in Figure 8. This reference implementation was selected for three main reasons, the first of which is the reference method has the best overall sensitivity, secondly the reference method is insensitive to AM noise [16], and lastly this method has a simplistic implementation as the majority of the required components were readily available.

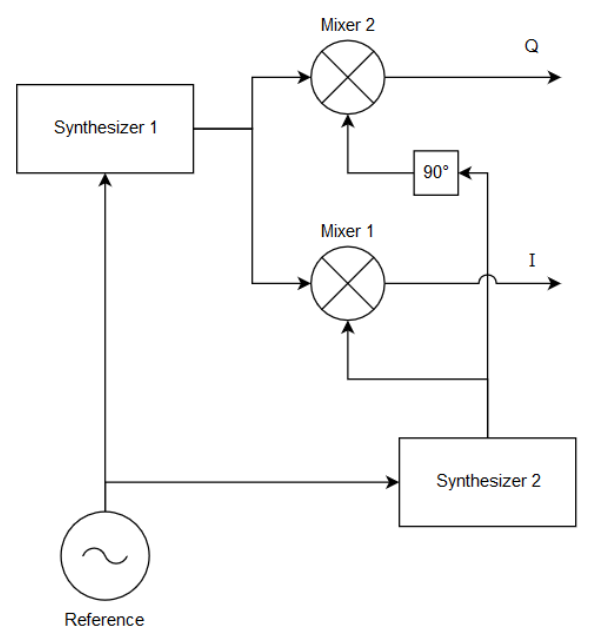

Figure 8: Phase Detector: Modified Reference Source Measurement Schematic 


\subsection{Linear Time-Invariant Systems and Signal Processing}

This section will discuss two assumptions made concerning properties of the proposed system which will be used during the system analysis, these are the linear system and time invariant system assumptions. This section will also discuss the digital signal processing techniques of windowing in the context of Fast Fourier Transforms (FFT), decimation, which will be implemented during measurement of the system, and IQ balancing, describing the benefits and drawbacks of these signal processing techniques.

\subsubsection{Time Invariant Systems}

Concerning time-invariance; systems can be broken into two categories; time invariant systems and time variant systems. A system that is time invariant can be described as a system where the output for a given input is not time dependant. For a given input $\mathrm{x}(\mathrm{t})$ the system $\mathrm{H}[\mathrm{x}]$ will give the output $\mathrm{y}(\mathrm{t})$, this can be mathematically expressed as seen below in equation 10.

$$
y(t)=H[x(t)]
$$

For this system to be considered time invariant equation 11 must also hold true, where $\tau$ is a time offset. In order to test this property this system is given an input, with the output $\mathrm{y}(\mathrm{t})$ measured. Next the input is delayed an arbitrary amount and repeated, if it results in the same output the system is considered time invariant [18].

$$
y(t-\tau)=H[x(t-\tau)]
$$




\subsubsection{Linear Systems}

While systems can be subdivided by whether they are time variant they can also be divided into two other categories; linear, or non-linear systems. In order for a system to be considered linear it must follow the superposition principle. The superposition principle states that the output of a system, $y(t)$, given a weighted sum of multiple inputs, $x_{n}(\mathrm{t})$, must be equal to the weighted sum of the individual outputs, this can be expressed mathematically as seen below in equation 12 [18].

$$
y(t)=H\left[a_{1} x_{1}(t)+a_{2} x_{2}(t)\right]=a_{1} H\left[x_{1}(t)\right]+a_{2} H\left[x_{2}(t)\right]
$$

The superposition principle can be broken into two properties of linear systems; the multiplicative property, and additive property. The multiplicative property states that the output of the system given an input multiplied by a scalar must be equal to the output of the system multiplied by that scalar, given a non-scalar multiplied input. This can be shown by simplifying 12 by choosing $a_{2}$ to be zero, this simplifies equation into equation 13 seen below.

$$
a_{1} y(t)=H\left[a_{1} x_{1}(t)\right]=a_{1} H\left[x_{1}(t)\right]
$$

The additive property of linear systems states that the output of a system given a sum of inputs is equal to the sum of outputs if the system is given the inputs individually. In order to demonstrate this mathematically choose $a_{1}$ and $a_{2}$ to be equal to 1 , the result can be seen in equation 14 below.

$$
y_{1}(t)+y_{2}(t)=H\left[x_{1}(t)\right]+H\left[x_{2}(t)\right]=H\left[x_{1}(t)+x_{2}(t)\right]
$$


From equation 13 it can be seen that if $a_{1}$ is set to 0 then the output of the system will also be zero. If the system is relaxed and a zero input results in a non-zero output, or the system does not follow the additive or multiplicative properties previously discussed the system is said to be non-linear [18].

\subsubsection{Spectral Leakage and Windowing}

A common method of converting a time domain signal into the frequency domain is through the use of the Fourier transform, specifically through the use of FFT algorithms. This takes a sampled time domain signal and converts it into a frequency domain signal. A major assumption that FFT uses is that the time and frequency domain are circular topologies, implying that the beginning and end of sampled the signal are connected. This assumption holds true if the signal is sampled an integer number of signal periods, however if a non-integer number of periods is sampled it causes a discontinuity within the signal. This discontinuity creates high frequency content that did not exist in the original time domain signal, these high frequency components are then aliased. Upon inspection of the FFT spectrum this aliasing causes the original signal to spread to nearby frequencies, this phenomenon is referred to as spectral leakage [19].

In order to combat this spectral leakage a technique called windowing can be implemented. Windowing refers to a process of reducing the discontinuities that cause spectral leakage, this is done by multiplying the sampled time series of the signal by a window of

equal length. This multiplication weights the middle portions of the time sampled signal higher, therefore it causes the signal to slowly approach zero at both the start and end of the time series. An example of a window being applied to a time domain signal can be seen below in Figure 9. 


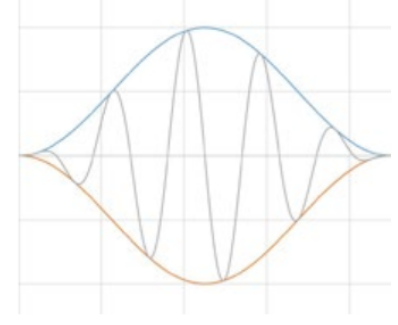

Figure 9: Applying Window to Signal [19]

As the signal approaches zero on either side the previous discontinuity that caused the spectral leakage is significantly reduced. If no window is applied to the time series then a rectangular window is implicitly applied as each sample is weighted equally. This rectangular window creates very large side lobes which are only $13 \mathrm{~dB}$ below the main peak of the signal. This creates a very narrow main lobe, however as previously mentioned it also causes spectral leakage, by applying a more sophisticated window to the time series the side lobes can be significantly reduced at the cost increasing the width of the main lobe [20]. For example, Figure 10 shows the original sampled spectrum with only the implicit rectangular window applied. By applying a non-rectangular window the spectrum can be changed to Figure 11, where the side lobe attenuation and main lobe widening can be observed.

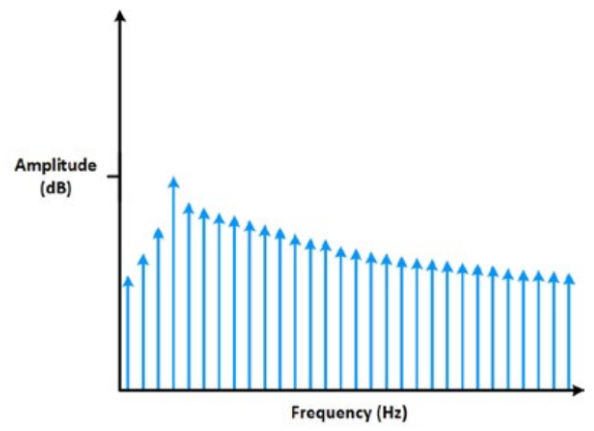

Figure 10: Original Spectrum of Arbitrary Signal [19] 


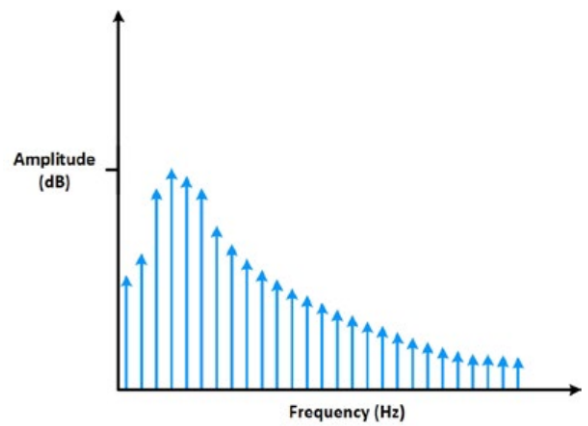

Figure 11: Windowed Spectrum of Arbitrary Signal [19]

There are a large number of weighting methods that could accomplish this windowing, however the window that will be discussed and implemented within this thesis is the DolphChebyshev, also referred to as a Chebyshev, or Dolph window. As the measurements for this system will be made very close to the main tone the Dolph-Chebyshev window was selected, due to the fact it has the minimum main lobe width for a given side lobe attenuation [21]. The window transform can be written as seen in equation 15 , where $\mathrm{M}$ is window length, $\beta$ is defined in equation 16 , and $\mathrm{A}$ is the amount of side lobe attenuation desired in $\mathrm{dB}$ [21].

$$
\begin{gathered}
W\left(\omega_{k}\right)=\frac{\cos \left[M \cos ^{-1}\left(\beta \cos \left(\frac{\pi k}{M}\right)\right)\right]}{\cosh \left[M \cosh { }^{-1}(\beta)\right]} \text { for } 0 \leq k \leq M-1 \\
\beta=\cosh \left(\frac{\cosh ^{-1}\left(10^{\frac{A}{20}}\right)}{N}\right)
\end{gathered}
$$

The window function for the Dolph-Chebyshev window in the time domain, w(n), can then be determined by taking the inverse Discrete Fourier Transform of the window transform $W\left(\omega_{k}\right)$, which is then multiplied by the time sampled series to apply the window [21]. 


\subsubsection{Decimation and Quantization Noise Reduction}

While Decimation is sometimes used exchangeably with downsampling, for the purposes of this thesis it will refer to the two step process of low-pass filtering, and subsequently downsampling a time sampled signal [22]. To understand the purpose for the low-pass filtering first downsampling must be discussed. Downsampling is the process of taking an over-sampled signal and taking every M sample to create a new time sampled signal, as seen in equation 17. This changes the effective sampling rate for the signal, the new sampling rate can be calculated as seen in equation 18 , where $\mathrm{M}$ is the downsampling factor, and $f_{s}$ is the sampling frequency.

$$
\begin{gathered}
x_{\text {new }}(m)=x_{\text {old }}(M m) \\
f_{\text {s,new }}=\frac{f_{\text {s,old }}}{M}
\end{gathered}
$$

As the downsampling process decreases the sampling frequency of the sampled sequence if there was higher frequency content in the signal it may be aliased, in order to avoid this the signal is low-pass filtered prior to downsampling. The low-pass filter is typically set to have a corner frequency at the new bandwidth of interest described in equation 19, which is also the new nyquist frequency.

$$
B^{\prime}=\frac{f_{s, n e w}}{2}
$$

While it initially seems like decimation does nothing but remove higher frequency content it has multiple applications. For the purposes of this thesis it will be used to reduce the quantization noise spectral density, and increase the effective Signal to Noise Ratio (SNR) 
of the analog to digital converter (ADC). The quantization noise power for a given ADC can be calculated using equation 20 , where $\mathrm{A}$ is the full range of the analog signal being converted, and $\mathrm{n}$ is the number of bits the ADC has for resolution [23].

$$
P_{Q N}=\sigma_{q}^{2}=\frac{A^{2}}{12\left(2^{2 n}\right)}
$$

The power spectral density (PSD) of the quantization noise can be calculated by dividing the noise power by the sampling frequency, resulting in the density seen in Figure 12.

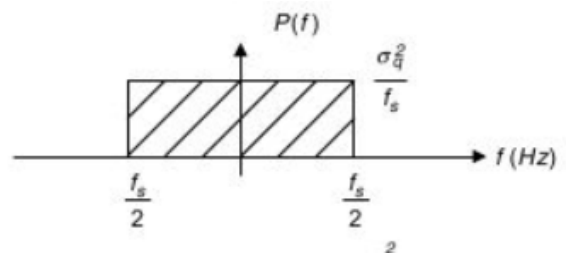

Figure 12: Spectrum of Quantization Noise for Typical ADC [23]

As the PSD of the quantization noise is inversely proportional to the sampling frequency increasing the sampling frequency, or oversampling the signal, results in the same total noise power, but a reduced power spectral density as seen in Figure 13.

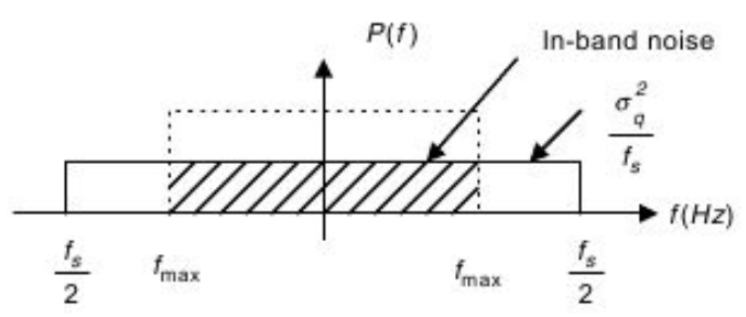

Figure 13: Spectrum of Quantization Noise for Oversampled Decimated ADC [23]

Therefore if the original signal is oversampled, and subsequently decimated it will have the original PSD of the higher sampling frequency, effectively increasing the number of bits 
the resolution of the ADC possess by reducing the quantization noise at the frequency of interest. This new effective number of bits can be calculated using equation 21 , where $f_{s}$ is the sampling rate for the oversampled signal, $f_{\max }$ is the maximum frequency of the analog signal, $\mathrm{m}$ is the number of bits per sample for the oversampled signal, and $\mathrm{n}$ is the effective number of bits of resolution [23].

$$
n=m+0.5\left(\log _{2}\left(\frac{f_{s}}{2 f_{\max }}\right)\right)
$$

\subsubsection{IQ Balancing}

As the system will use an IQ mixer the problem of IQ imbalance, which has three contributing problems; DC offset, IQ amplitude imbalance, and IQ quadrature error, must be addressed. This IQ imbalance is caused due to the differences in the I and Q signal paths throughout the mixer. In order to correct for IQ imbalance three corrections must be made, the correction factors are created by taking an uncorrected complex waveform, sampled at a frequency equal to an integer sub-multiple of the sample rate and having an integer number of cycles. These correction factors can then be applied to other signals that no longer fulfill the requirements to calculate them, such as baseband signals. The first error, the DC offset, is represented when measuring IQ data as a constant offset in both the I and Q channels. As it is a constant offset across all measurements it can be measured by simply taking the mean of the signal as seen below in equation 22 , where $\mathrm{N}$ is the total number of terms, and $z_{n}$ is the term of the complex signal at index value $\mathrm{n}$.

$$
D C_{\text {offset }}=\bar{z}=\frac{1}{N} \sum_{n=1}^{N} z_{n}
$$

This mean is simply subtracted from each measurement in order to correct for it as seen below in equation 23 , where $z_{\text {Original }}$ is the complex signal initially measured. 


$$
z_{\text {new } 1}=z_{\text {Original }}-D C_{\text {offset }}
$$

The second correction to be made is the IQ amplitude imbalance, this is done through a gain correction. This is done by taking the standard deviation of the real and imaginary components as seen in equations 24 and 25 , where $\mathrm{x}$ represents the real component of the complex signal, and y represents the imaginary component of the complex signal.

$$
\begin{gathered}
\sigma_{\text {real }}=\sqrt{\frac{1}{N} \sum_{n=1}^{N}\left[x_{n}-\bar{x}\right]^{2}} \\
\sigma_{\text {imaginary }}=\sqrt{\frac{1}{N} \sum_{n=1}^{N}\left[y_{n}-\bar{y}\right]^{2}}
\end{gathered}
$$

Once the standard deviation of each component is calculated the gain correction term is then calculated as seen below in equation 26 .

$$
G_{\text {Corr }}=\frac{\sigma_{\text {real }}}{\sigma_{\text {imaginary }}}
$$

This determines the ratio of the gain of the Q channel compared to the I channel. The signal is once again corrected by multiplying the imaginary component of the signal by this correction factor as seen below in equation 27 , where $x_{n e w 1}$ and $y_{n e w 1}$ are the real and imaginary components of the complex waveform with DC offset corrected respectively.

$$
z_{n e w 2}=x_{n e w 1}+i\left(G_{C o r r} * y_{n e w 1}\right)
$$

The final correction is for the IQ quadrature error, this correction value is calculated 
as seen below in equations 28 through 30 , where $x_{n}$ and $y_{n}$ are the real and imaginary components of the complex waveform with DC offset and amplitude imbalance corrected respectively.

$$
\begin{gathered}
P_{1}=\sum_{n=1}^{N}\left[x_{n} * y_{n}\right] \\
P_{2}=\sum_{n=1}^{N}\left[x_{n}^{2}\right] \\
Q_{\text {Corr }}=\frac{P_{1}}{P_{2}}
\end{gathered}
$$

This correction factor is then applied as seen below in equation 31 in order to determine the final corrected signal $z_{\text {new } 3}$, where $x_{n e w 2}$ and $y_{n e w 2}$ are the real and imaginary components of the complex waveform with DC offset and amplitude imbalance corrected respectively.

$$
z_{\text {new } 3}=x_{n e w 2}+i\left(\frac{y_{n e w 2}-Q_{C o r r} * y_{n e w 2}}{\sqrt{1-Q_{\text {Corr }}^{2}}}\right)
$$




\subsection{UHF RFID and Self-Interference}

This section will discuss the theory and applications of RFID, a common modulation method used in the implementation of RFID, and a limitation of the full-duplex architecture used for RFID implementation called self-interference. As mentioned earlier RFID refers to a group of technologies which through the use of electromagnetic waves can identify a physical object [4]. While RFID has typically been used in a somewhat small set of applications, such as supply chain management or toll ticketing for roads, ever improving technology could allow for new applications. Possible new applications such as airline baggage tracking, patient information visibility in hospitals, or vehicle identification information [24].

There are a number of ways an RFID system can be implemented with regards to components, modulation methods, frequency ranges, etc. Typically however, the system generates a signal at a given frequency, it is transmitted to the RFID tag, which modulates data onto the signal and transmits it back to the reader, which then down mixes the signal to baseband to interpret the data. Additionally RFID systems utilize passive or active tags, active tags have their own power supply in order to transmit information to the reader, while passive tags are powered by a continuous wave from the reader [4]. Due to the fact that passive tags do not require an additional power source and can instead be powered remotely they are generally used for applications with a large number of tags, such as warehouse or supply chain management.

\subsubsection{On-Off Shift Keying and Pulse Interval Encoding}

In order to transmit data a baseband signal is modulated with the carrier signal. A simplified example of how an RFID link with a simple modulation architecture can be seen below in Figure 14. The transmitted signal hits the receiver, or tag, and depending on the load attached, in this case a short or open circuit, back scatters a different signal. When the short circuit is attached the signal is back-scattered to the original source, when an open 
circuit is attached no current flows in the receiver and therefore no signal is back-scattered, this is a limited method of amplitude modulation referred to as On-Off keying (OOK).

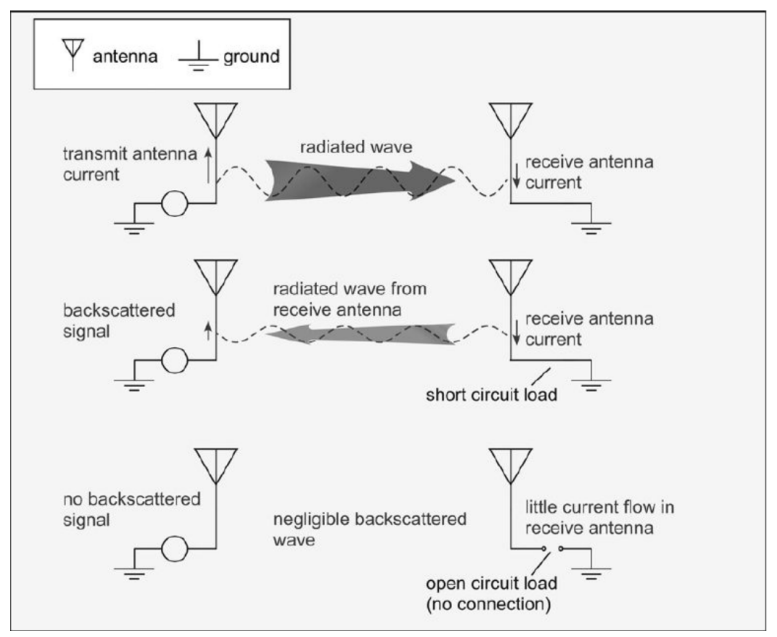

Figure 14: Simplified RFID Link [4]

The time domain representation of this modulation can be expressed mathematically as seen below in equation 32 , where $s_{x}$ denotes the symbol number, $\mathrm{A}$ is the amplitude of the signal, and $f_{c}$ is the baseband frequency [25] and can be visually represented as seen below in Figure 15.

$$
\begin{array}{r}
s_{1}(t)=A \cos \left(2 \pi f_{c} t\right), \quad A=1, \quad 0 \leq t \leq T \\
s_{2}(t)=0, \quad A=0, \quad 0 \leq t \leq T
\end{array}
$$




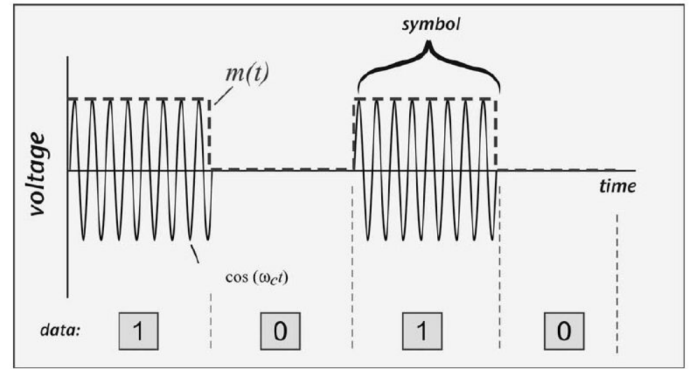

Figure 15: On-Off Keyed Signal [4]

This however creates a problem with passive tags when using OOK, if the transmitted data remains a ' 0 ', the tag receives no power from the continuous wave, and as such the tag will turn off. In order to supply power to the tag, even when the data remains '0', a common method of solving this problem is coding the data prior to modulation. An example of this coding is a method called pulse-interval encoding, where a ' 1 ' is defined as a short off pulse following a long on pulse, and a ' 0 ' is coded as a short off pulse following a short on pulse. This allows for $50 \%$ of the peak power to reach the tag for a long string of zeroes, and $63 \%$ for an equally mixed signal of zeroes and ones [4].

\subsubsection{Self-Interference}

As RFID systems utilize full duplex architectures they both transmit and receive at the same time. While this allows the RFID system to function as intended it also creates a self interference issue. There are multiple paths through which the transmitted signal reaches the receiver, the most common is leakage through the isolator seen in Figure 22 [4], however it can also reach the receiver due to reflections along the transmission path due to impedance mismatch [26]. For clarity the sum of all of these interfering signals will be referred to as the self-interference signal beyond this point. In an ideal scenario this wouldn't impact the received signal from the tag as only the carrier tone would leak through, potentially causing saturation issues in the receiver, however as the data from the tag is modulated at 
some frequency offset from the carrier it would not interfere with the data. Unfortunately as previously mentioned oscillators, and therefore the transmit signal, have a phase noise profile associated with them. Therefore if the self-interference signal is a large enough power, or the phase noise relative to the carrier is high enough at the modulated frequency offset it will reduce the sensitivity of the receiver, potentially stopping a tag signal from being received. 


\subsection{Current Solutions to Self-Interference}

As self-interference is a system limiting factor there are already a number of potential solutions proposed and patented. For example, using an adaptive circuit to cancel selfinterference signals [27], or through the use of a frequency immunization converter to separate the unwanted transmit signal from the desired received signal [28]. Research of cancellation methods continues in order to improve the magnitude or ease of implementation of cancellation methods. This section will introduce and discuss the various degrees of success of the designs proposed within recent publications.

\subsubsection{Code-domain Spread Spectrum Modulation}

A recent paper written by Zhenqi Chen utilizes code-domain spread spectrum modulation (CSSM) and an integrated on-chip duplexer with a tunable load impedance in order to reject in-band jamming and self-interference signals, the proposed system level block diagram of the circuit can be seen below in Figure 16 [29].

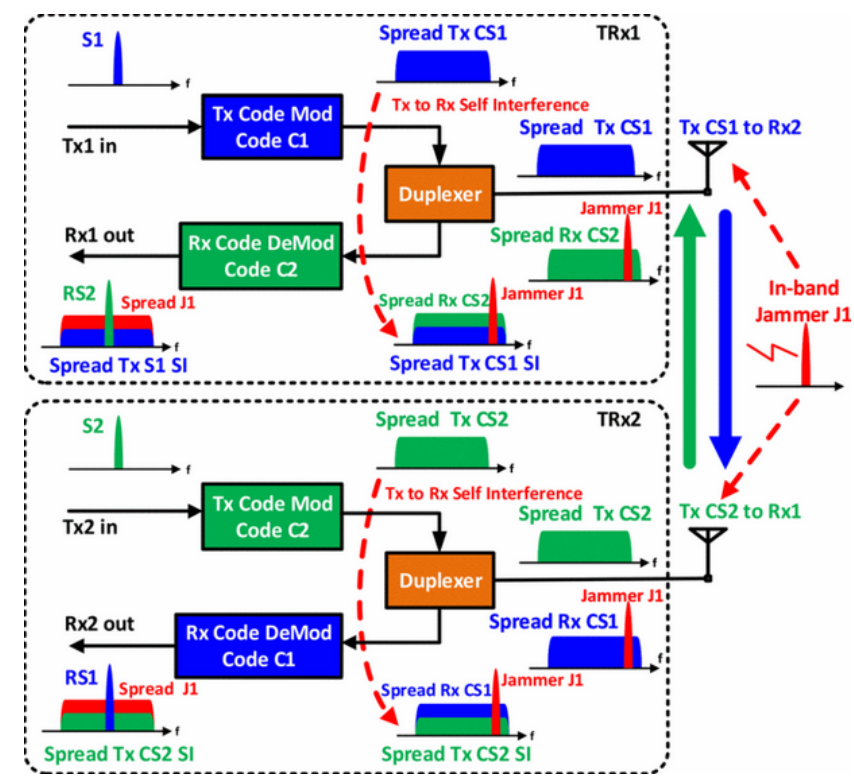

Figure 16: CSSM and On-Chip Duplexer System Level Diagram [29] 
From the diagram it can be seen that the transmitter and receiver utilize two separate codes, causing only the intended signal to be demodulated at the receiver. In order to implement the CSSM a butterfly switch was placed in front of both the Power Amplifier (PA) on the transmit portion, and the Low Noise Amplifier (LNA) on the receiver end as seen below in Figure 17. The load tuner utilizes mostly on-chip components with the exception a single off-chip 1 bit tunable inductor.

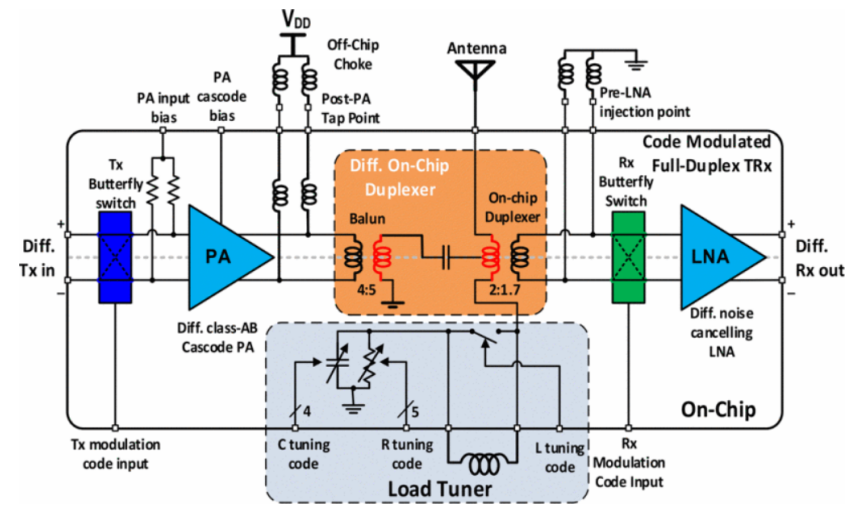

Figure 17: CSSM full-duplex transceiver front-end architecture [29]

Through the use of both the CSSM and well tuned duplexer a total self-interference cancellation of $51 \mathrm{~dB}$ was measured across a $1 \mathrm{MHz}$ bandwidth [29].

\subsubsection{Highly Integrated Tunable Duplexing Transceiver}

Another recently published paper written by Dong Yang evaluated the use of a fully integrated wide-band active duplexing transceiver with baseband noise-cancelling duplexing LNAs, the system for which can be seen below in Figure 18. A series of four pairs of mixer switches were used with LO inputs of various phase, with each pair set $180^{\circ}$ out of phase, in order to provide a low noise figure and large input impedance tuning range [30]. A duplexing LNA is then used after each mixing switch pair, followed by a baseband amplifier. 


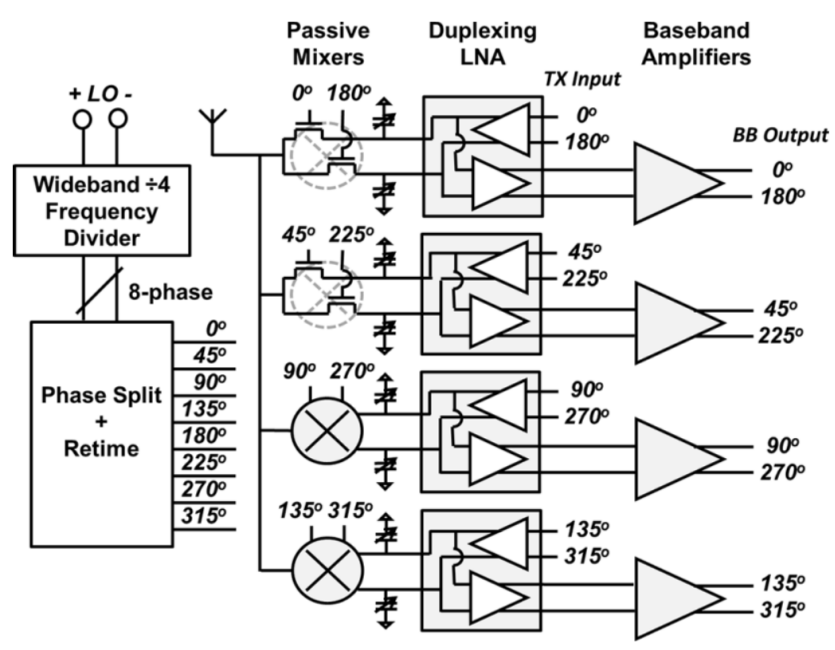

Figure 18: System Diagram of Transceiver [30]

The duplexing LNAs utilize two differential pairs, as seen below in Figure 19. By connecting the middle two transistors into a differential pair they can be biased separately from the top two transistors through the use of the current source connected to the source of the transistors. In order to combat the effects of the baseband sampling capacitors at higher baseband frequencies, denoted as $C_{B B}$ in Figure 19, a digitally variable capacitor CL was implemented. Lastly in order to counteract the effects of imaginary impedances of the antenna at RF frequencies a feed forward network was added on both the I and Q paths. 


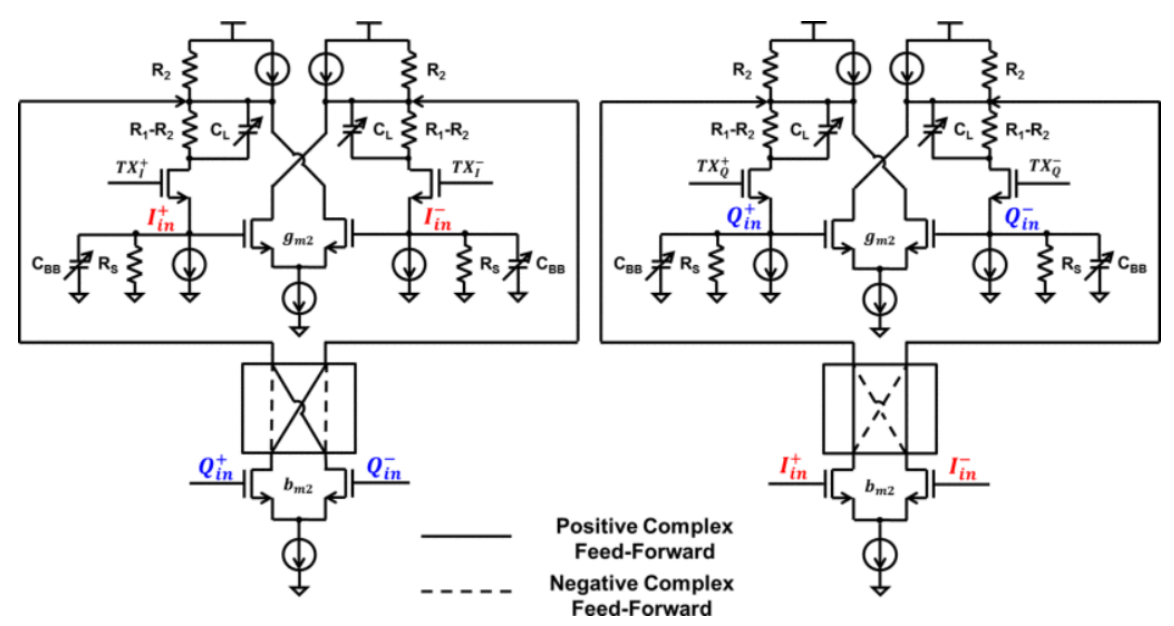

Figure 19: Circuit Diagram of Duplexing LNA [30]

Through the use of the active duplexing transceiver system a self-interference cancellation of approximately $30 \mathrm{~dB}$ was measured between the transmitter and receiver [30].

\subsubsection{Magnetic-Free N-path-Filter Non-Reciprocal Circulator and Base-band Self-Interference Cancellation}

Within a paper written by Jin Zhou and Negar Reiskarimian an N-path-filter magnetic free non-reciprocal circulator using simultaneous base-band Self-Interference Cancellation was proposed and measured. The proposed system, seen below in Figure 20, is defined as linear time-varying due to the non-reciprocal nature of the circulator. In order to perform selfinterference cancellation the base-band transmit signal is tapped, the amplitude and phase of the signal is altered through the use of vector modulators and is then injected at the input of the transimpedance amplifiers in the receiver. 


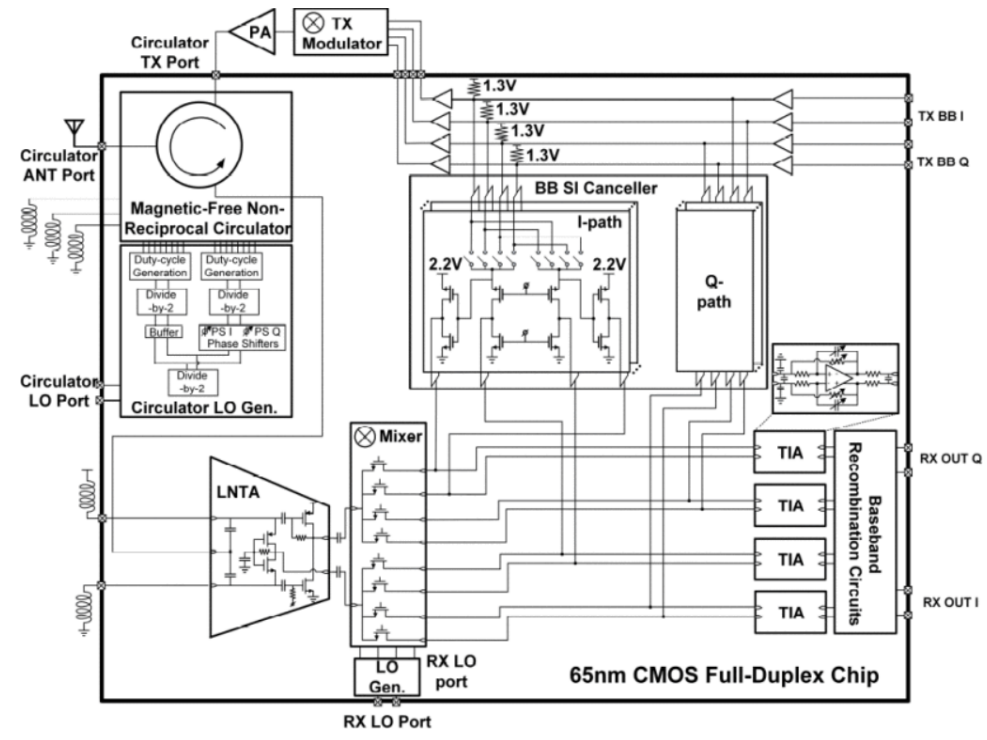

Figure 20: N-path-Filter Non-Reciprocal Circulator System Diagram [31]

A non-reciprocal circulator will avoid the inherent loss of a reciprocal network, to this end the N-path filter was implemented with a $\frac{3}{4} \lambda$ ring as seen below in Figure 21. Assuming the value of $\mathrm{N}$ is large and the $\mathrm{N}$-path $\mathrm{RC}$ time-constant is also large the implementation of the N-path filter causes the system to have low loss, and more importantly the S21 and S12 parameters of the network to be equal in magnitude but $180^{\circ}$ out of phase at $\pm 90^{\circ}$ [31]. The $\frac{3}{4} \lambda$ transmission line provides a $+90^{\circ}$ phase shift, which when combining with the $-90^{\circ}$ phase shift of the filter causes constructive interference, but when combining with the $+90^{\circ}$ phase shift of the filter it creates destructive interference, therefore signals will only propagate in the clockwise direction. 


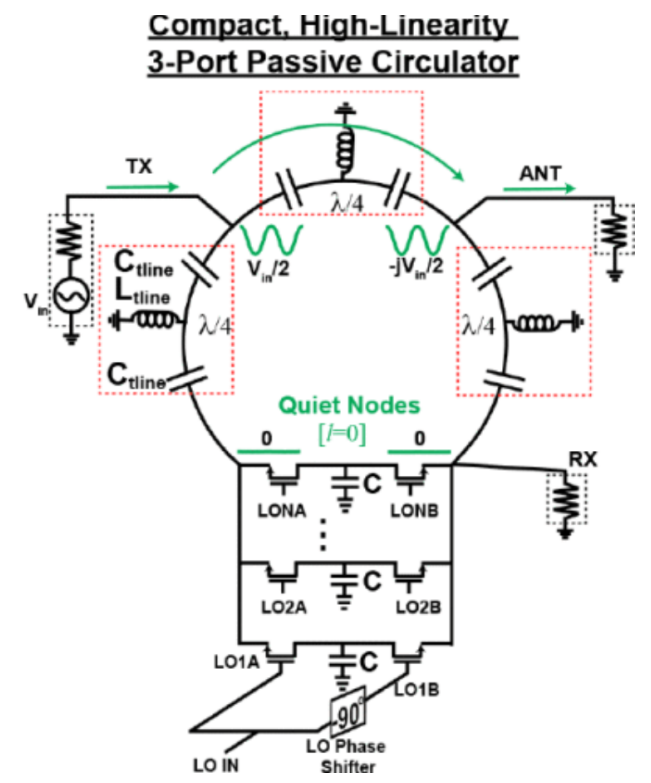

Figure 21: N-path-Filter Non-Reciprocal Circulator System Diagram [Modified from [31]]

When measured the combination of the non-reciprocal circulator and base-band cancellation achieved a self-interference cancellation of $42 \mathrm{~dB}$ over a $12 \mathrm{MHz}$ signal bandwidth. 


\section{Mixer Phase Noise Cancellation System Analysis}

This chapter will discuss the theory and analysis of the mixer phase noise cancellation system. The system utilizes an oscillator, a $-90^{\circ}$ phase shifter, a linear time-invariant system, two mixers, and two band-pass filters. It will be shown that through the use of this system the phase noise cancellation of an oscillator can be calculated according to equation 33 .

$$
C_{P N}=\left|e^{-i \theta_{0}}-\frac{s_{21-}}{s_{210}} e^{-i \theta_{-}}\right|^{2}+\left|e^{-i \theta_{0}}-\frac{s_{21+}}{s_{210}} e^{-i \theta_{+}}\right|^{2}
$$

An example application system, that the system used to perform this cancellation will represent, can be seen in Figure 22 below and is a typical Radio Frequency Identification (RFID) transceiver system architecture. For the analysis presented the portion of the system surrounded by the dotted line is assumed to be a linear time-invariant system. In order for this assumption to be accurate, the RFID area must not change rapidly, and the transceiver must be designed such that it operates linearly. The proposed system can be utilized in full

duplex transceiver architectures such as RFID or Radio Detection And Ranging (RADAR) systems with some limitation. 


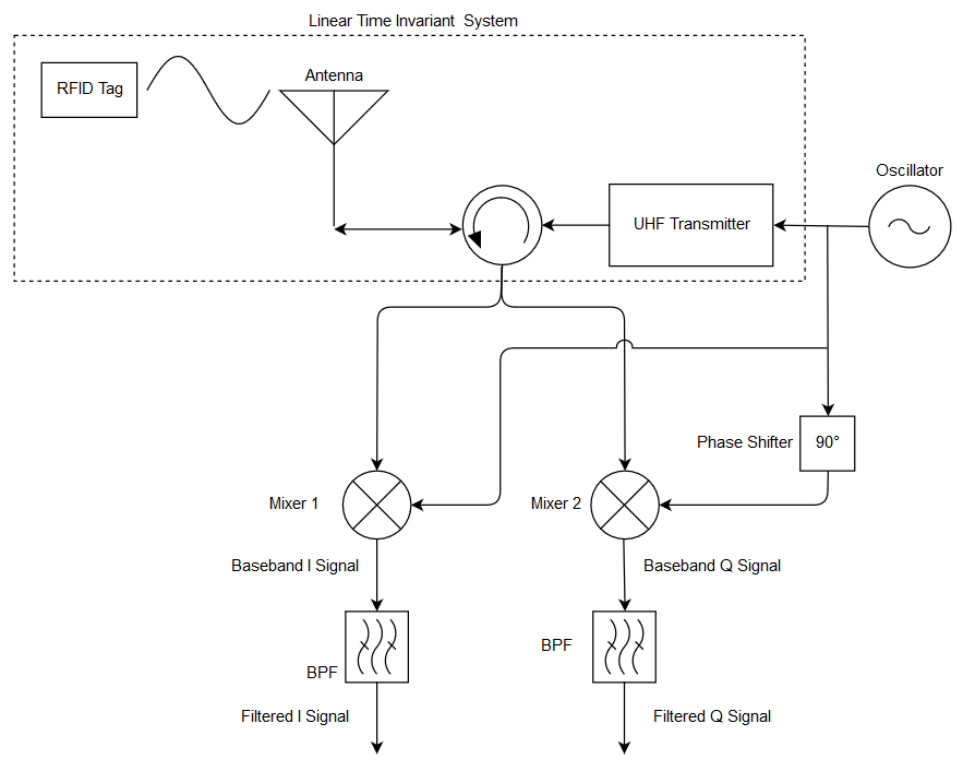

Figure 22: Mixer Phase Noise Cancellation Circuit Diagram

The example signal used for this system is an oscillator with an associated phase noise process. The input oscillator signal will be passed through a linear time-invariant system and transmitted and will also be used to down-convert the received signal in an IQ mixer, with the local oscillator tone being the originally inputted signal. The action of passing the signal through a linear time-invariant system will cause some phase and amplitude response, dependant on the system used. This delay then effectively causes the original oscillator output to be mixed with a previous output from the oscillator. Using both the original oscillator output and the $-90^{\circ}$ phase shifted signal to mix with the delayed signal both I and Q base-band signals are created, and simultaneously phase noise cancellation occurs. Therefore the proposed system provides a method to cancel the oscillator phase noise in full duplex transceivers provided the system can be approximated to be linear and time-invariant. The I and Q signals can then be individually passed through band-pass filters to significantly attenuate any DC tones and any other interfering signals outside of the band of interest. 


\subsection{Determining Initial Inputs}

The Oscillator seen below in Figure 23 outputs a complex signal, a $(\mathrm{t})$ described in equation 34 , where $\phi(\mathrm{t})$ is the phase noise process of the oscillator. The generated signal a $(\mathrm{t})$ will be used to create two addition signals as seen in 23 . The signal $\mathrm{b}(\mathrm{t})$ will be created by passing $\mathrm{a}(\mathrm{t})$ through a linear time invariant signal with an amplitude and phase response denoted by $\mathrm{H}(\omega)$. A third signal $\mathrm{c}(\mathrm{t})$ will be created by passing a(t) through a $-90^{\circ}$ phase shift. The resulting $\mathrm{b}(\mathrm{t})$ signal will then be mixed with $\mathrm{a}(\mathrm{t})$ and $\mathrm{c}(\mathrm{t})$ in order to create the $\mathrm{I}$ and $\mathrm{Q}$ signals respectively. Both the I and Q signal will subsequently passed through a band-pass filter in order to attenuate their higher frequency components.

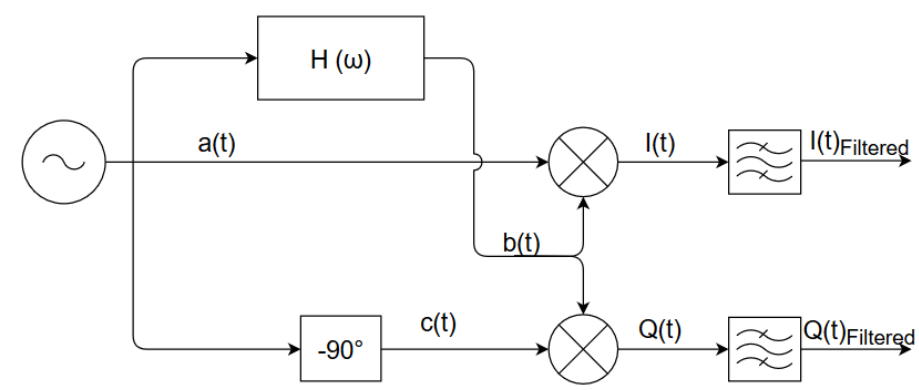

Figure 23: System Diagram

$$
\begin{aligned}
a(t) & =\mathrm{e}^{\mathrm{i}\left(\omega_{c} t+\varphi(t)\right)} \\
& =\left(\cos \left(\omega_{c} t\right)+\mathrm{i} \sin \left(\omega_{c} t\right)\right)(\cos (\phi(t))+\mathrm{i} \sin (\phi(t)))
\end{aligned}
$$

\subsubsection{Input $\mathrm{a}(\mathrm{t})$}

In order to simplify the analysis of the system it is assumed that the analysis is taking place at a distance away from the carrier such that the amplitude of the phase noise process $\phi(t)$ 
$<<1$. Once this simplification is implemented equation 35 is the result.

$$
\left.a(t)=\left(\cos \left(\omega_{c} t\right)+\mathrm{i} \sin \left(\omega_{c} t\right)\right)(1+\mathrm{i} \phi(t))\right)
$$

Lastly it is assumed that the phase noise process at a single frequency $\omega_{n}$ can be approximated by a sinusoidal signal with amplitude $a_{n}$ giving the signal seen in equation 36 .

$$
a(t)=\left(\cos \left(\omega_{c} t\right)+\mathrm{i} \sin \left(\omega_{c} t\right)\right)\left(1+\mathrm{i} \mathrm{a}_{\mathrm{n}} \sin \left(\omega_{n} t\right)\right)
$$

Once expanded this yields equation 37 .

$$
\begin{aligned}
& a(t)=\cos \left(\omega_{c} t\right)+\mathrm{i} \sin \left(\omega_{c} t\right)+\frac{\mathrm{a}_{\mathrm{n}} \cos \left(\omega_{c} t+\omega_{n} t\right)}{2}-\frac{\mathrm{a}_{\mathrm{n}} \cos \left(\omega_{c} t-\omega_{n} t\right)}{2} \\
& +\frac{i a_{n} \sin \left(\omega_{c} t+\omega_{n} t\right)}{2}-\frac{i a_{n} \sin \left(\omega_{c} t-\omega_{n} t\right)}{2}
\end{aligned}
$$

Next the real part of this signal is taken as one of the inputs to the mixers, this can be seen below in equation 38 .

$$
\operatorname{Re}\{a(t)\}=\cos \left(\omega_{c} t\right)+\frac{\mathrm{a}_{\mathrm{n}} \cos \left(\omega_{c} t+\omega_{n} t\right)}{2}-\frac{\mathrm{a}_{\mathrm{n}} \cos \left(\omega_{c} t-\omega_{n} t\right)}{2}
$$

\subsubsection{Input $b(t)$}

The real part of a(t) as seen in equation 38 is then passed through a linear time-invariant system $\mathrm{H}(\omega)$, with an amplitude response of $h_{0}$ at $\omega_{c}, h_{+}$at $\omega_{c}+\omega_{n}, h_{-}$at $\omega_{c}-\omega_{n}$, and phase response of $\theta_{0}$ at $\omega_{c}, \theta_{+}$at $\omega_{c}+\omega_{n}, \theta_{-}$at $\omega_{c}-\omega_{n}$ giving the result seen in equation 39

$$
\begin{aligned}
\operatorname{Re}\{b(t)\}= & \mathrm{h}_{0} \cos \left(\theta_{0}+\omega_{c} t\right)-\frac{\mathrm{h}_{-} \mathrm{a}_{\mathrm{n}} \cos \left(\theta_{-}+\omega_{c} t-\omega_{n} t\right)}{2} \\
& +\frac{\mathrm{h}_{+} \mathrm{a}_{\mathrm{n}} \cos \left(\theta_{+}+\omega_{c} t+\omega_{n} t\right)}{2}
\end{aligned}
$$




\subsubsection{Input $\mathrm{c}(\mathrm{t})$}

To calculate the input $\mathrm{c}(\mathrm{t})$ the original signal a(t) is simply phase shifted by $-90^{\circ}$, or multiplied by -i resulting in equation 40 .

$$
\begin{aligned}
c(t)= & \sin \left(\omega_{c} t\right)+\frac{\mathrm{a}_{\mathrm{n}} \sin \left(\omega_{c} t+\omega_{n} t\right)}{2}-\frac{\mathrm{a}_{\mathrm{n}} \sin \left(\omega_{c} t-\omega_{n} t\right)}{2} \\
& -\mathrm{i} \cos \left(\omega_{c} t\right)-\frac{\mathrm{i} \mathrm{a}_{\mathrm{n}} \cos \left(\omega_{c} t+\omega_{n} t\right)}{2}+\frac{\mathrm{i} \mathrm{a}_{\mathrm{n}} \cos \left(\omega_{c} t-\omega_{n} t\right)}{2}
\end{aligned}
$$

Once again the real part of this signal was taken and results in equation 41 .

$$
\operatorname{Re}\{c(t)\}=\sin \left(\omega_{c} t\right)+\frac{\mathrm{a}_{\mathrm{n}} \sin \left(\omega_{c} t+\omega_{n} t\right)}{2}-\frac{\mathrm{a}_{\mathrm{n}} \sin \left(\omega_{c} t-\omega_{n} t\right)}{2}
$$

\subsection{Mixing Signals and Baseband Power}

\subsubsection{Output I(t)}

The output $\mathrm{I}(\mathrm{t})$ can be determined by multiplying the real part of $\mathrm{a}(\mathrm{t})$, and the real part of $\mathrm{b}(\mathrm{t})$ as given earlier in equation 38 and equation 39 respectively, this results in equation 43 .

$$
I(t)=\operatorname{Re}\{a(t)\} \cdot \operatorname{Re}\{b(t)\}
$$




$$
\begin{aligned}
I(t)= & \frac{\mathrm{h}_{0} \cos \left(\theta_{0}+2 \omega_{c} t\right)}{2}+\frac{\mathrm{h}_{0} \cos \left(\theta_{0}\right)}{2} \\
& +\frac{\mathrm{h}_{-} \mathrm{a}_{\mathrm{n}}{ }^{2} \cos \left(\theta_{-}+2 \omega_{c} t-2 \omega_{n} t\right)}{8} \\
& +\frac{\mathrm{h}_{+} \mathrm{a}_{\mathrm{n}}{ }^{2} \cos \left(\theta_{+}+2 \omega_{c} t+2 \omega_{n} t\right)}{8} \\
& +\frac{\mathrm{h}_{-} \mathrm{a}_{\mathrm{n}}{ }^{2} \cos \left(\theta_{-}\right)}{8}+\frac{\mathrm{h}_{+} \mathrm{a}_{\mathrm{n}}{ }^{2} \cos \left(\theta_{+}\right)}{8} \\
& -\frac{\mathrm{h}_{0} \mathrm{a}_{\mathrm{n}} \cos \left(\theta_{0}+\omega_{n} t\right)}{4}+\frac{\mathrm{h}_{0} \mathrm{a}_{\mathrm{n}} \cos \left(\theta_{0}-\omega_{n} t\right)}{4} \\
& -\frac{\mathrm{h}_{-} \mathrm{a}_{\mathrm{n}} \cos \left(\theta_{-}-\omega_{n} t\right)}{4}+\frac{\mathrm{h}_{+} \mathrm{a}_{\mathrm{n}} \cos \left(\theta_{+}+\omega_{n} t\right)}{4} \\
& +\frac{\mathrm{h}_{0} \mathrm{a}_{\mathrm{n}} \cos \left(\theta_{0}+2 \omega_{c} t+\omega_{n} t\right)}{4}-\frac{\mathrm{h}_{0} \mathrm{a}_{\mathrm{n}} \cos \left(\theta_{0}+2 \omega_{c} t-\omega_{n} t\right)}{4} \\
& -\frac{\mathrm{h}_{-} \mathrm{a}_{\mathrm{n}} \cos \left(\theta_{-}+2 \omega_{c} t-\omega_{n} t\right)}{4}+\frac{\mathrm{h}_{+} \mathrm{a}_{\mathrm{n}} \cos \left(\theta_{+}+2 \omega_{c} t+\omega_{n} t\right)}{4} \\
& -\frac{\mathrm{h}_{-} \mathrm{a}_{\mathrm{n}}{ }^{2} \cos \left(\theta_{-}+2 \omega_{c} t\right)}{8}-\frac{\mathrm{h}_{+} \mathrm{a}_{\mathrm{n}}{ }^{2} \cos \left(\theta_{+}+2 \omega_{c} t\right)}{8} \\
& -\frac{\mathrm{h}_{-} \mathrm{a}_{\mathrm{n}}{ }^{2} \cos \left(\theta_{-}-2 \omega_{n} t\right)}{8}-\frac{\mathrm{h}_{+} \mathrm{a}_{\mathrm{n}}{ }^{2} \cos \left(\theta_{+}+2 \omega_{n} t\right)}{8}
\end{aligned}
$$

This signal is then passed through a band-pass filter in order to attenuate all signals at significantly higher frequencies than $\omega_{n}$. In order to simplify the expression further all terms with an exponent of $a_{n}^{2}$ or higher are assumed to be negligible as it is assumed that $a_{n}<1$, therefore $a_{n}^{2}<<1$. Removing the affected terms gives equation 44 .

$$
\begin{aligned}
I(t)= & \frac{h_{0} \mathrm{a}_{\mathrm{n}} \cos \left(\theta_{0}-\omega_{\mathrm{n}} t\right)}{4}-\frac{h_{0} \mathrm{a}_{\mathrm{n}} \cos \left(\theta_{0}+\omega_{\mathrm{n}} t\right)}{4} \\
& -\frac{\mathrm{h}_{-} \mathrm{a}_{\mathrm{n}} \cos \left(\theta_{-}-\omega_{\mathrm{n}} t\right)}{4}+\frac{\mathrm{h}_{+} \mathrm{a}_{\mathrm{n}} \cos \left(\theta_{+}+\omega_{\mathrm{n}} t\right)}{4}
\end{aligned}
$$

\subsubsection{Output $\mathrm{Q}(\mathrm{t})$}

The output $\mathrm{Q}(\mathrm{t})$ can be determined by multiplying the real part of $\mathrm{c}(\mathrm{t})$, and the real part of $\mathrm{b}(\mathrm{t})$ as given earlier in equation 41 and equation 39 respectively, this results in equation 46.

$$
Q(t)=\operatorname{Re}\{c(t)\} \cdot \operatorname{Re}\{b(t)\}
$$




$$
\begin{aligned}
Q(t)= & \frac{\mathrm{h}_{0} \sin \left(\theta_{0}+2 \omega_{c} t\right)}{2}-\frac{\mathrm{h}_{0} \sin \left(\theta_{0}\right)}{2} \\
& +\frac{\mathrm{h}_{-} \mathrm{a}_{n}{ }^{2} \sin \left(\theta_{-}+2 \omega_{c} t-2 \omega_{n} t\right)}{8} \\
& +\frac{\mathrm{h}_{+} \mathrm{a}_{\mathrm{n}}{ }^{2} \sin \left(\theta_{+}+2 \omega_{c} t+2 \omega_{n} t\right)}{8} \\
& -\frac{\mathrm{h}_{-} \mathrm{a}_{n}{ }^{2} \sin \left(\theta_{-}\right)}{8}-\frac{\mathrm{h}_{+} \mathrm{a}_{\mathrm{n}}{ }^{2} \sin \left(\theta_{+}\right)}{8} \\
& +\frac{\mathrm{h}_{0} \mathrm{a}_{\mathrm{n}} \sin \left(\theta_{0}+\omega_{n} t\right)}{4}-\frac{\mathrm{h}_{0} \mathrm{a}_{\mathrm{n}} \sin \left(\theta_{0}-\omega_{n} t\right)}{4} \\
& +\frac{\mathrm{h}_{-} \mathrm{a}_{\mathrm{n}} \sin \left(\theta_{-}-\omega_{n} t\right)}{4}-\frac{\mathrm{h}_{+} \mathrm{a}_{\mathrm{n}} \sin \left(\theta_{+}+\omega_{n} t\right)}{4} \\
& +\frac{\mathrm{h}_{0} \mathrm{a}_{\mathrm{n}} \sin \left(\theta_{0}+2 \omega_{c} t+\omega_{n} t\right)}{4}-\frac{\mathrm{h}_{0} \mathrm{a}_{\mathrm{n}} \sin \left(\theta_{0}+2 \omega_{c} t-\omega_{n} t\right)}{4} \\
& -\frac{\mathrm{h}_{-} \mathrm{a}_{\mathrm{n}} \sin \left(\theta_{-}+2 \omega_{c} t-\omega_{n} t\right)}{4}+\frac{\mathrm{h}_{+} \mathrm{a}_{\mathrm{n}} \sin \left(\theta_{+}+2 \omega_{c} t+\omega_{n} t\right)}{4} \\
& -\frac{\mathrm{h}_{-} \mathrm{a}_{n}{ }^{2} \sin \left(\theta_{-}+2 \omega_{c} t\right)}{8}-\frac{\mathrm{h}_{+} \mathrm{a}_{\mathrm{n}}{ }^{2} \sin \left(\theta_{+}+2 \omega_{c} t\right)}{8} \\
& +\frac{\mathrm{h}_{-} \mathrm{a}_{\mathrm{n}}{ }^{2} \sin \left(\theta_{-}-2 \omega_{n} t\right)}{8}+\frac{\mathrm{h}_{+} \mathrm{a}_{\mathrm{n}}{ }^{2} \sin \left(\theta_{+}+2 \omega_{n} t\right)}{8}
\end{aligned}
$$

Similarly to the $\mathrm{I}(\mathrm{t})$ signal, $\mathrm{Q}(\mathrm{t})$ is passed through a band-pass filter attenuating all frequency components above $\omega_{n}$ and all terms with an exponent of $a_{n}^{2}$ or higher are assumed to be negligible. Eliminating the affected frequency components gives equation 47 .

$$
\begin{aligned}
Q(t)= & \frac{\mathrm{h}_{0} \mathrm{a}_{\mathrm{n}} \sin \left(\theta_{0}+\omega_{\mathrm{n}} t\right)}{4}-\frac{\mathrm{h}_{0} \mathrm{a}_{\mathrm{n}} \sin \left(\theta_{0}-\omega_{\mathrm{n}} t\right)}{4} \\
& +\frac{\mathrm{h}_{-} \mathrm{a}_{\mathrm{n}} \sin \left(\theta_{-}-\omega_{\mathrm{n}} t\right)}{4}-\frac{\mathrm{h}_{+} \mathrm{a}_{\mathrm{n}} \sin \left(\theta_{+}+\omega_{\mathrm{n}} t\right)}{4}
\end{aligned}
$$

\subsubsection{Mixer Output and Phase Noise Cancellation}

Now that both the I and Q outputs have been determined it is possible to determine the total mixer output and subsequently the base-band power of the signal. First the I and Q terms from equation 44 and equation 47 respectively are combined to create the complex 
signal $\mathrm{m}(\mathrm{t})$ seen in equation 48.

$$
\begin{aligned}
m(t) & =\frac{h_{0} \mathrm{a}_{\mathrm{n}} \cos \left(\theta_{0}-\omega_{\mathrm{n}} t\right)}{4}-\frac{h_{0} \mathrm{a}_{\mathrm{n}} \cos \left(\theta_{0}+\omega_{\mathrm{n}} t\right)}{4} \\
& -\frac{\mathrm{h}_{-} \mathrm{a}_{\mathrm{n}} \cos \left(\theta_{-}-\omega_{\mathrm{n}} t\right)}{4}+\frac{\mathrm{h}_{+} \mathrm{a}_{\mathrm{n}} \cos \left(\theta_{+}+\omega_{\mathrm{n}} t\right)}{4} \\
+i\left(\frac{\mathrm{h}_{0} \mathrm{a}_{\mathrm{n}} \sin \left(\theta_{0}+\omega_{\mathrm{n}} t\right)}{4}-\frac{\mathrm{h}_{0} \mathrm{a}_{\mathrm{n}} \sin \left(\theta_{0}-\omega_{\mathrm{n}} t\right)}{4}\right. & \\
& \left.+\frac{\mathrm{h}_{-} \mathrm{a}_{\mathrm{n}} \sin \left(\theta_{-}-\omega_{\mathrm{n}} t\right)}{4}-\frac{\mathrm{h}_{+} \mathrm{a}_{\mathrm{n}} \sin \left(\theta_{+}+\omega_{\mathrm{n}} t\right)}{4}\right) \\
& =\frac{\mathrm{a}_{\mathrm{n}}}{4}\left[h_{0} \cos \left(\theta_{0}-\omega_{\mathrm{n}} t\right)-h_{0} \cos \left(\theta_{0}+\omega_{\mathrm{n}} t\right)\right. \\
& -\mathrm{h}_{-} \cos \left(\theta_{-}-\omega_{\mathrm{n}} t\right)+\mathrm{h}_{+} \cos \left(\theta_{+}+\omega_{\mathrm{n}} t\right) \\
& +i\left(\mathrm{~h}_{0} \sin \left(\theta_{0}+\omega_{\mathrm{n}} t\right)-\mathrm{h}_{0} \sin \left(\theta_{0}-\omega_{\mathrm{n}} t\right)\right. \\
& \left.\left.+\mathrm{h}_{-} \sin \left(\theta_{-}-\omega_{\mathrm{n}} t\right)-\mathrm{h}_{+} \sin \left(\theta_{+}+\omega_{\mathrm{n}} t\right)\right)\right] \\
& =\frac{\mathrm{a}_{\mathrm{n}}}{4}\left[h_{0} \cos \left(\omega_{\mathrm{n}} t-\theta_{0}\right)-h_{0} \cos \left(\omega_{\mathrm{n}} t+\theta_{0}\right)\right. \\
& -\mathrm{h}_{-} \cos \left(\omega_{\mathrm{n}} t-\theta_{-}\right)+\mathrm{h}_{+} \cos \left(\omega_{\mathrm{n}} t+\theta_{+}\right) \\
& +i\left(\mathrm{~h}_{0} \sin \left(\omega_{\mathrm{n}} t+\theta_{0}\right)+\mathrm{h}_{0} \sin \left(\omega_{\mathrm{n}} t-\theta_{0}\right)\right. \\
& \left.\left.-\mathrm{h}_{-} \sin \left(\omega_{\mathrm{n}} t-\theta_{-}\right)-\mathrm{h}_{+} \sin \left(\omega_{\mathrm{n}} t+\theta_{+}\right)\right)\right] \\
&
\end{aligned}
$$

These are then converted into multiple exponential signals as seen in equation 49, where $H_{x}=h_{x} \cdot e^{-i \theta_{x}}$.

$$
m(t)=\frac{a_{n}}{4}\left[H_{0} \mathrm{e}^{\mathrm{iwn} t}-H_{0} \mathrm{e}^{-\mathrm{iwn} t}-\mathrm{H}_{-} \mathrm{e}^{\mathrm{iwn} t}+\mathrm{H}_{+} \mathrm{e}^{-\mathrm{iwn} t}\right]
$$

As the base-band power of an exponential signal is calculated using the conjugate of the signal and the signal itself [32] the conjugate of $\mathrm{m}(\mathrm{t})$ was determined in equation 50 , where $H_{x}^{*}=\operatorname{conjugate}\left(H_{x}\right)$.

$$
m(t)^{*}=\frac{a_{n}}{4}\left[H_{0}^{*} \mathrm{e}^{-\mathrm{iwn} t}-H_{0}^{*} \mathrm{e}^{\mathrm{iwn} t}-\mathrm{H}_{-}^{*} \mathrm{e}^{-\mathrm{iwn} t}+\mathrm{H}_{+}^{*} \mathrm{e}^{\mathrm{iwn} t}\right]
$$


Equation 49 and equation 50 are then used to calculate the base-band power as seen below in equation 51.

$$
\begin{aligned}
P_{B B} & =m(t) * m(t)^{*} \\
& =\frac{a_{n}^{2}}{16}\left[2 H_{0} H_{0}^{*}-H_{0} \mathrm{H}_{-}^{*}-H_{0}^{*} \mathrm{H}_{-}-H_{0} \mathrm{H}_{+}^{*}-H_{0}^{*} \mathrm{H}_{+}+\mathrm{H}_{-} \mathrm{H}_{-}^{*}\right. \\
& +\mathrm{H}_{+} \mathrm{H}_{+}^{*}-H_{0} H_{0}^{*} \mathrm{e}^{-\mathrm{i} 2 \mathrm{wn} t}-H_{0} H_{0}^{*} \mathrm{e}^{\mathrm{i} 2 \mathrm{wn} t} \\
& +H_{0} \mathrm{H}_{-}^{*} \mathrm{e}^{-\mathrm{i} 2 \mathrm{wn} t}+H_{0}^{*} \mathrm{H}_{-} \mathrm{e}^{\mathrm{i} 2 \mathrm{wn} t}+H_{0} \mathrm{H}_{+}^{*} \mathrm{e}^{\mathrm{i} 2 \mathrm{wn} t} \\
& \left.+H_{0}^{*} \mathrm{H}_{+} \mathrm{e}^{-\mathrm{i} 2 \mathrm{wn} t}-\mathrm{H}_{-} \mathrm{H}_{+}^{*} \mathrm{e}^{\mathrm{i} 2 \mathrm{wn} t}-\mathrm{H}_{-}^{*} \mathrm{H}_{+} \mathrm{e}^{-\mathrm{i} 2 \mathrm{wn} t}\right]
\end{aligned}
$$

Next, the non-DC terms are removed from equation 51 giving equation 52 .

$$
\begin{aligned}
P B B & =\frac{a_{n}^{2}}{16}\left[2 H_{0} H_{0}^{*}-H_{0} \mathrm{H}_{-}^{*}-H_{0}^{*} \mathrm{H}_{-}-H_{0} \mathrm{H}_{+}^{*}-H_{0}^{*} \mathrm{H}_{+}+\mathrm{H}_{-} \mathrm{H}_{-}^{*}\right. \\
& \left.+\mathrm{H}_{+} \mathrm{H}_{+}^{*}\right]
\end{aligned}
$$

Equation 52 is then factored resulting in equation 53 below.

$$
P B B=\frac{a_{n}^{2}}{16}\left[\left(H_{0}-\mathrm{H}_{-}\right)\left(H_{0}^{*}-\mathrm{H}_{-2}\right)+\left(H_{0}-\mathrm{H}_{+}\right)\left(H_{0}^{*}-\mathrm{H}_{+2}\right)\right]
$$

Next, the identity $|x|^{2}=x \cdot \operatorname{conjugate}(x)$ is applied to equation 53 , which results in the result for the base-band power of the signal in equation 54 .

$$
P B B=\frac{a_{n}^{2}}{16}\left[\left|H_{0}-\mathrm{H}_{-}\right|^{2}+\left|H_{0}-\mathrm{H}_{+}\right|^{2}\right]
$$

Each term is then expanded into its original form as seen in equation 55.

$$
P B B=\frac{a_{n}^{2}}{16}\left[\left|h_{210} e^{-i \theta_{0}}-h_{21-} e^{-i \theta_{-}}\right|^{2}+\left|h_{210} e^{-i \theta_{0}}-h_{21+} e^{-i \theta_{+}}\right|^{2}\right]
$$


In order to calculate only the cancellation of the phase noise, and exclude the attenuation of the signal due to the system itself as it will not change the phase noise relative to the carrier, the amplitude response of the signal is factored out as seen below in equation 56 .

$$
P B B=\frac{a_{n}^{2}}{16}\left[h_{0}^{2}\right]\left[\left|e^{-i \theta_{0}}-\frac{h_{-}}{h_{0}} e^{-i \theta_{-}}\right|^{2}+\left|e^{-i \theta_{0}}-\frac{h_{+}}{h_{0}} e^{-i \theta_{+}}\right|^{2}\right]
$$

Therefore the phase noise cancellation of the system can then be calculated as shown in equation 57.

$$
C_{P N}=\left|e^{-i \theta_{0}}-\frac{h_{-}}{h_{0}} e^{-i \theta_{-}}\right|^{2}+\left|e^{-i \theta_{0}}-\frac{h_{+}}{h_{0}} e^{-i \theta_{+}}\right|^{2}
$$

Therefore in order to maximize the resulting phase noise cancellation the system under test should be designed such that the phase and amplitude response are as uniform as possible across the noise bandwidth of interest. 


\section{Test System Implementation and Measurements}

This chapter will discuss the characterization and implementation of the previously described circuit. Following this it will provide measurements and discussion of the results, demonstrating the functionality of the circuit as well as it's typical performance. This chapter is composed of four sections: System Implementation, Component Characterization, Phase Noise Cancellation Measurements, and Cancellation Overview. For the purposes of all baseband measurements were taken as single-sideband, and a high input impedance PICOscope was used, 25 captures were taken and averaged in an attempt to reduce large noise outliers, a decimation factor of 50 was used in order to reduce the quantization noise of the PICOscope, and a sampling rate of $500 \mathrm{MS} / \mathrm{s}$ was used. For RF measurements a Rhode and Schwarz CMU 200 was used with a resolution bandwidth of $20 \mathrm{kHz}$.

\subsection{System Implementation}

This section will first discuss the implementation of the IQ mixer circuit and subsequently the overall system, as well as identify the various components used within them. The IQ mixer was built using the schematic seen below in Figure 24 . 


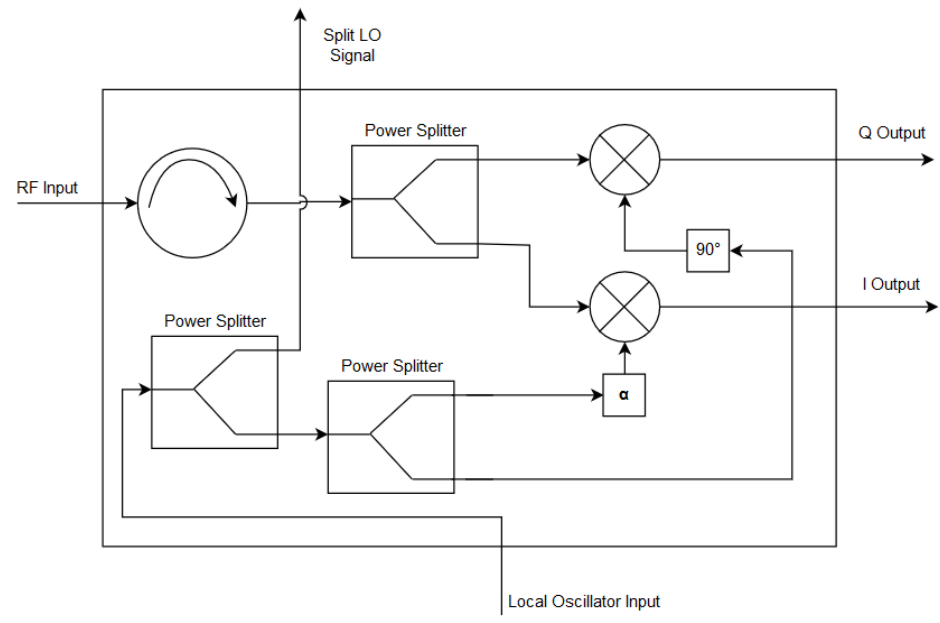

Figure 24: IQ Mixer Schematic

The ZFM-4H-S+ was used for both mixers, the power splitter used at the RF input and to split the LO input signal were two ZN2PD-20-S+, and lastly a ZX10Q-2-12-S+ power splitter was used for the power splitter and $90^{\circ}$ phase shifter at the LO input, all of these components were purchased from Mini-Circuits. In addition to these a phase shifter was added along the I channel LO signal path in order to address phase the phase imbalance due to the fact the power splitter did not create an exactly $90^{\circ}$ offset between its ports.

The RF and LO inputs to the system were constructed as seen below in Figure 25 and Figure 26 respectively. For both inputs a Version 4 TPI synthesizer and two CBP-915C band-pass filters were used. The TPI synthesizers do not contain self-filtering, therefore an external filter at the frequency of interest, in this case $915 \mathrm{MHz}$, was required. The RF input simply uses two cascaded band-pass filters in order to attenuate the harmonics of the signals. 


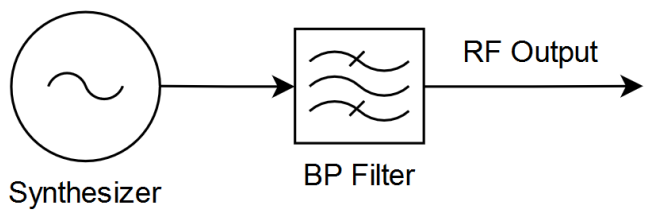

Figure 25: RF Input Schematic

The TPI synthesizers had a maximum output power setting of $10 \mathrm{dBm}$, therefore for the LO input an amplifier was required in order to supply the required $17 \mathrm{dBm}$ for the mixers, as seen below in Figure 26, therefore a ZHL-42 amplifier was used due to it's high linearity. In order to reduce the overall noise figure of the input circuit one band-pass filter was placed prior the amplifier in order to minimize non-linearity of the amplifier due to harmonics, and a second filter was placed after the amplifier in order to further attenuate any unwanted signals.

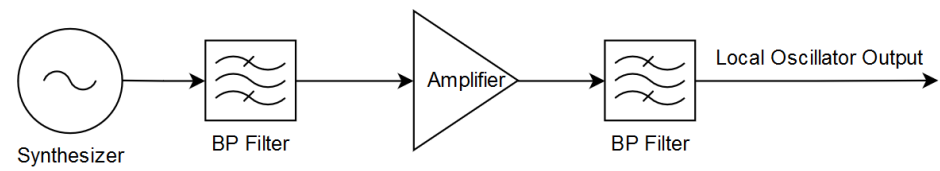

Figure 26: Local Oscillator Input Schematic

\subsection{Component Characterization}

This section will characterize the IQ Mixer implemented within the system, as well as characterize the phase noise profile of the synthesizers used in order to set a baseline with which to measure the results. It is comprised of four subsections: IQ Correction, IQ Mixer Gain Characterization, IQ Mixer Phase Characterization, and Synthesizer Phase Noise Characterization. 


\subsubsection{IQ Correction}

For the purposes of baseband measurements the previously discussed IQ correction was applied to each signal. For the gain characterization a $1 \mathrm{MHz}$ intermediate frequency was used, the IQ correction for each measurement was calculated from the data collected. In order to demonstrate the functionality of the correction figures 27 and 28 below show the time domain signal and frequency domain spectrum of the $1 \mathrm{MHz}$ signal before correction.

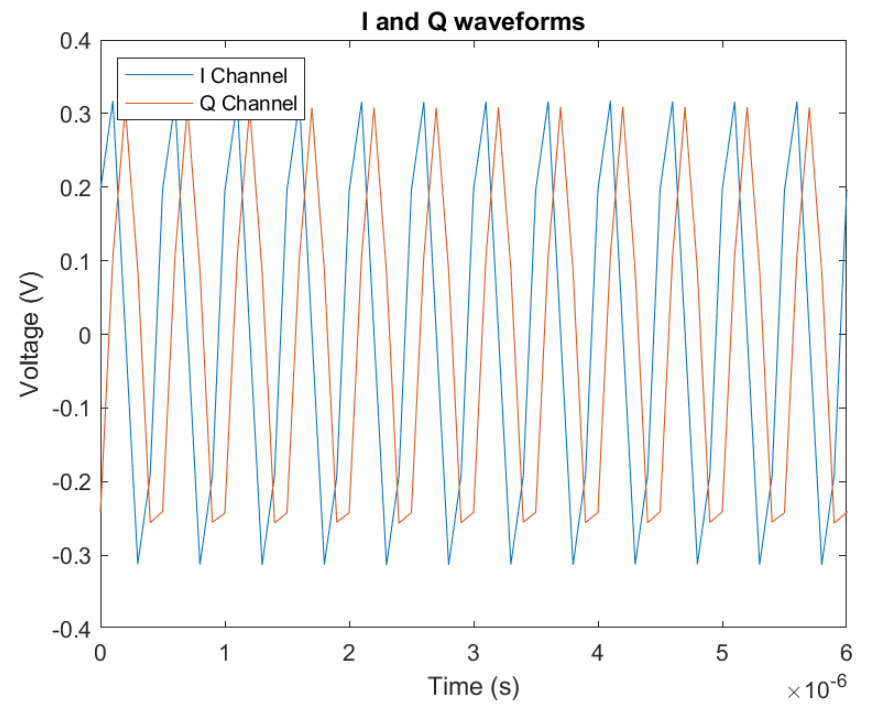

Figure 27: Time Domain $1 \mathrm{MHz}$ Signal Before Correction 


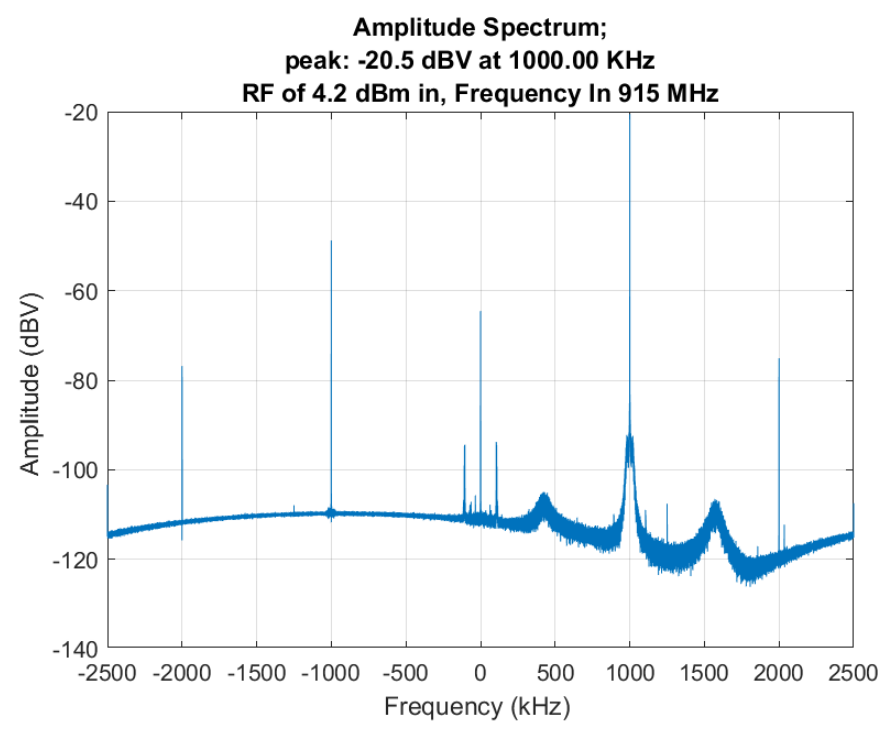

Figure 28: Frequency Domain $1 \mathrm{MHz}$ Signal Spectrum Before Correction

The IQ amplitude imbalance can be seen in the time domain signals. Additionally as the spectrum shown in Figure 28 is a complex spectrum it should not be symmetrical, therefore the signal appearing at $-1 \mathrm{MHz}$ is mainly due to IQ mismatch. The IQ correction factors were applied and the resulting time domain signal and frequency domain spectrum can be seen below in figures 29 and 30 respectively. 


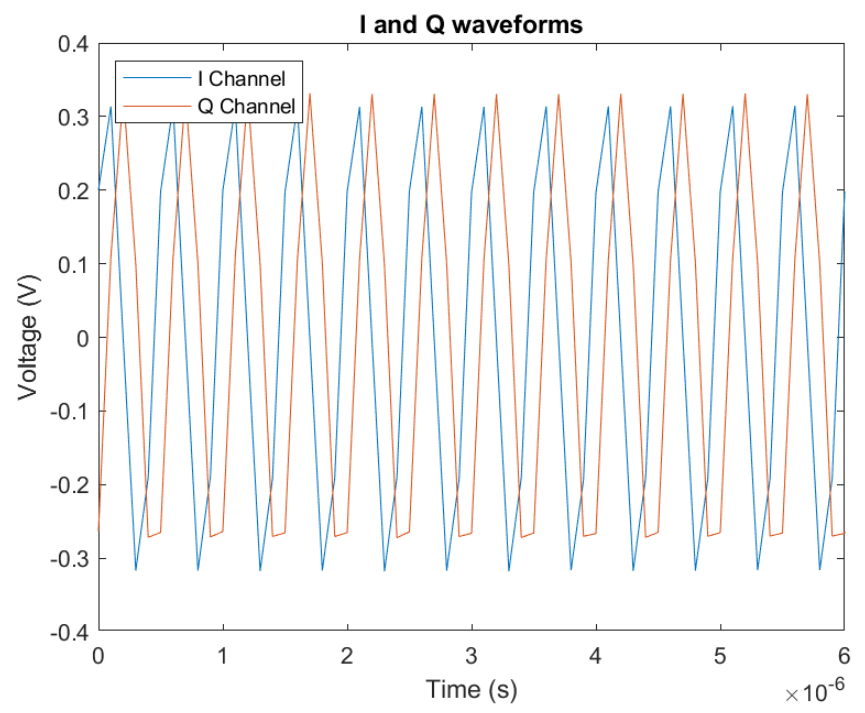

Figure 29: Time Domain $1 \mathrm{MHz}$ Signal After Correction

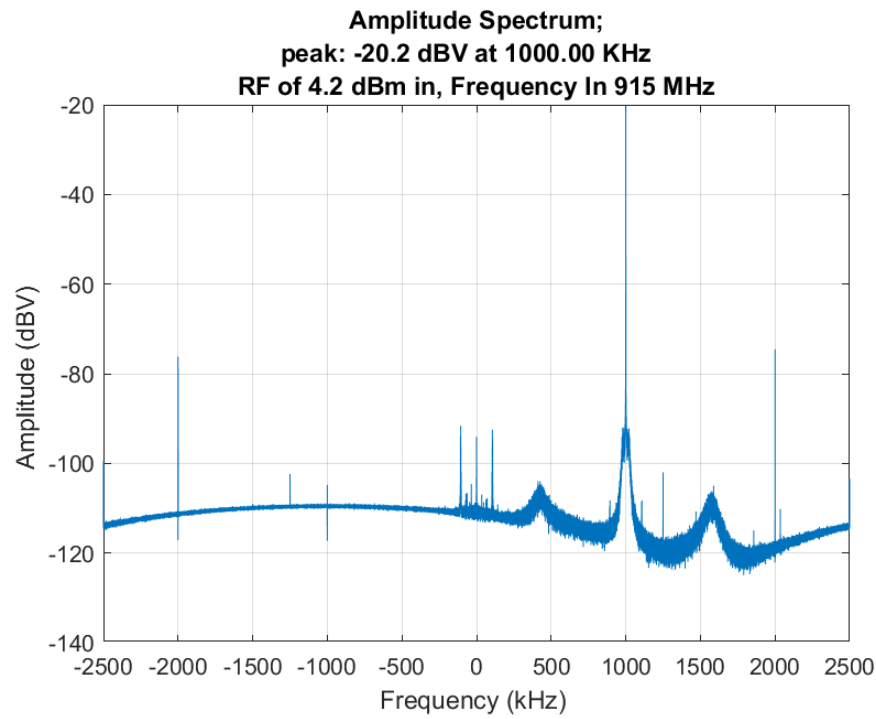

Figure 30: Frequency Domain $1 \mathrm{MHz}$ Signal Spectrum After Correction

The amplitude of the time domain signal can be seen to be significantly closer, however due to the sampling rate the exact peaks cannot be determined. Additionally the image 
that appeared at $-1 \mathrm{MHz}$ has dropped by approximately $50 \mathrm{~dB}$, and the $\mathrm{DC}$ component dropped by approximately $30 \mathrm{~dB}$. The phase correction will be demonstrated within the phase characterization subsection within this thesis.

\subsubsection{IQ Mixer Gain Characterization}

This subsection will discuss the circuit used in order to characterize the gain of the IQ mixer used within the system. The schematic of the system can be seen below in Figure 31, where two separate but identical synthesizers are used in order to create the LO and RF inputs.

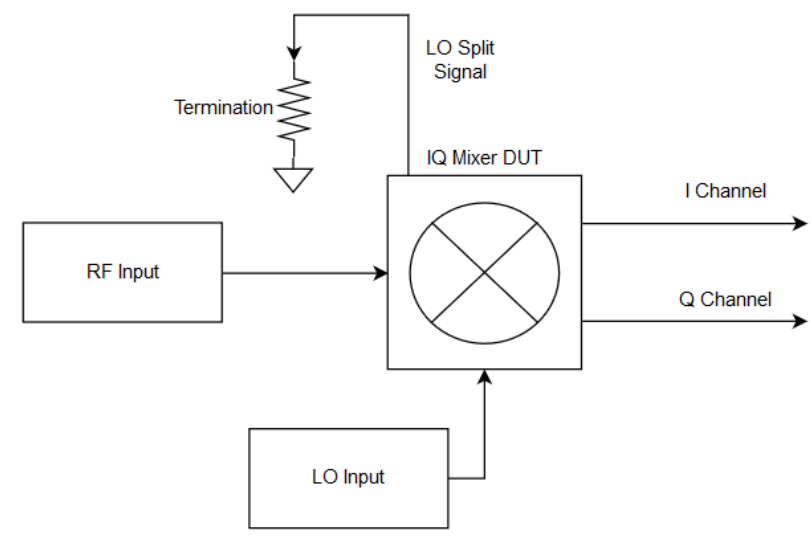

Figure 31: IQ Mixer Gain Characterization Schematic

The LO power was set such that the input power at the LO port of each discrete mixer within the IQ Mixer system was approximately $16.3 \mathrm{dBm}$ for both the original LO signal, the spectrum for which can be seen below in Figure 32, and for the $90^{\circ}$ phase shifted signal as seen below in Figure 33 . 


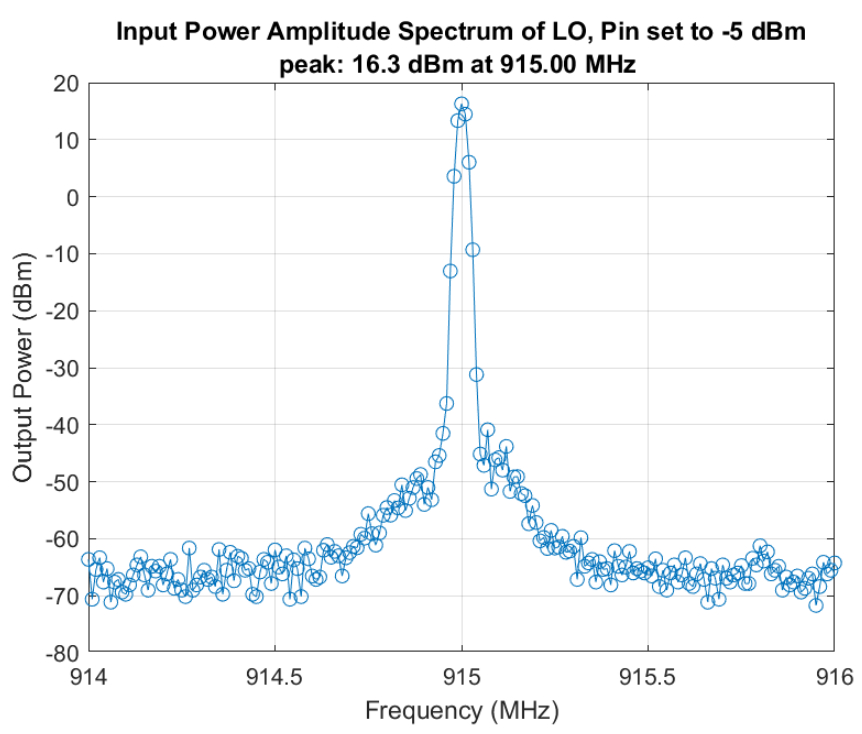

Figure 32: Mixer LO Input Spectrum

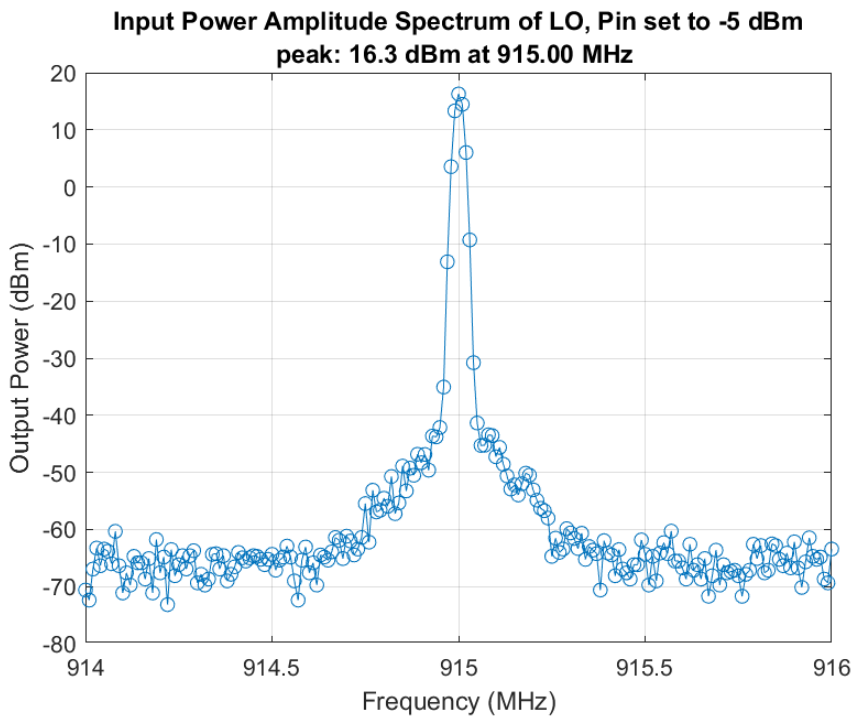

Figure 33: $90^{\circ}$ Phase-Shifted Mixer LO Input Spectrum

The RF input power was then swept on the synthesizer, resulting in an input range of $-10.1 \mathrm{dBm}$ to $8.2 \mathrm{dBm}$ at the IQ Mixer input port. The measured input and output power 
for each setting can be seen plotted below in Figure 34, the recorded data can be seen in Table 1, along with the calculated gain in units of (dBV out - $\mathrm{dBm}$ in).

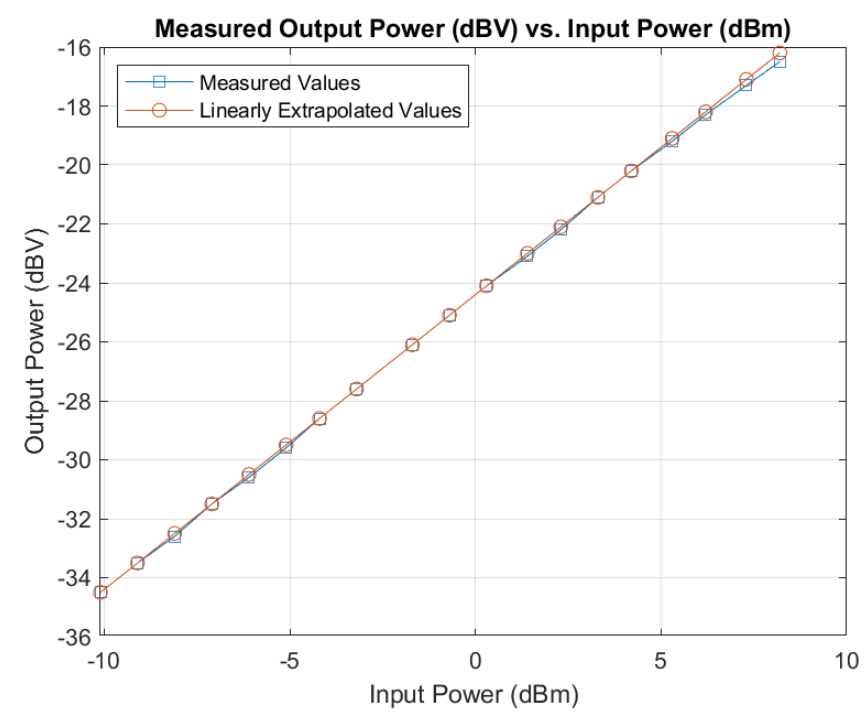

Figure 34: Output Power versus Input Power Of IQ Mixer

From the measurements it can be seen that the gain does slightly vary by $\pm 0.1 \mathrm{~dB}$ in the linear region, and that the system may begin to compress after the input power of $4.2 \mathrm{dBm}$, therefore a conservative limit for future measurements will be that the maximum allowable input power is $4.2 \mathrm{dBm}$ in order to ensure the system is operating in a linear fashion. 
Table 1: Gain Measurement Results of IQ Mixer

\begin{tabular}{|c|c|c||c|c|c|}
\hline$P_{\text {in }}(d B m)$ & $P_{\text {out }}(d B V)$ & Gain $(d B V / d B m)$ & $P_{\text {in }}(d B m)$ & $P_{\text {out }}(d B V)$ & Gain $(d B V / d B m)$ \\
\hline \hline 8.2 & -16.5 & -24.7 & -1.7 & -26.1 & -24.4 \\
\hline 7.3 & -17.3 & -24.6 & -3.2 & -27.6 & -24.4 \\
\hline 6.2 & -18.3 & -24.5 & -4.2 & -28.6 & -24.4 \\
\hline 5.3 & -19.2 & -24.5 & -5.1 & -29.6 & 24.5 \\
\hline 4.2 & -20.2 & -24.4 & -6.1 & -30.6 & -24.5 \\
\hline 3.3 & -21.1 & -24.4 & -7.1 & -31.5 & -24.4 \\
\hline 2.3 & -22.2 & -24.5 & -8.1 & -32.6 & -24.5 \\
\hline 1.4 & -23.1 & -24.5 & -9.1 & -33.5 & -24.4 \\
\hline 0.3 & -24.1 & -24.4 & -10.1 & -34.5 & -24.4 \\
\hline-0.7 & -25.1 & -24.4 & & & \\
\hline
\end{tabular}

The final ten data points were plotted again as seen in Figure 35 below in order to demonstrate beginning of compression of the IQ mixer. 


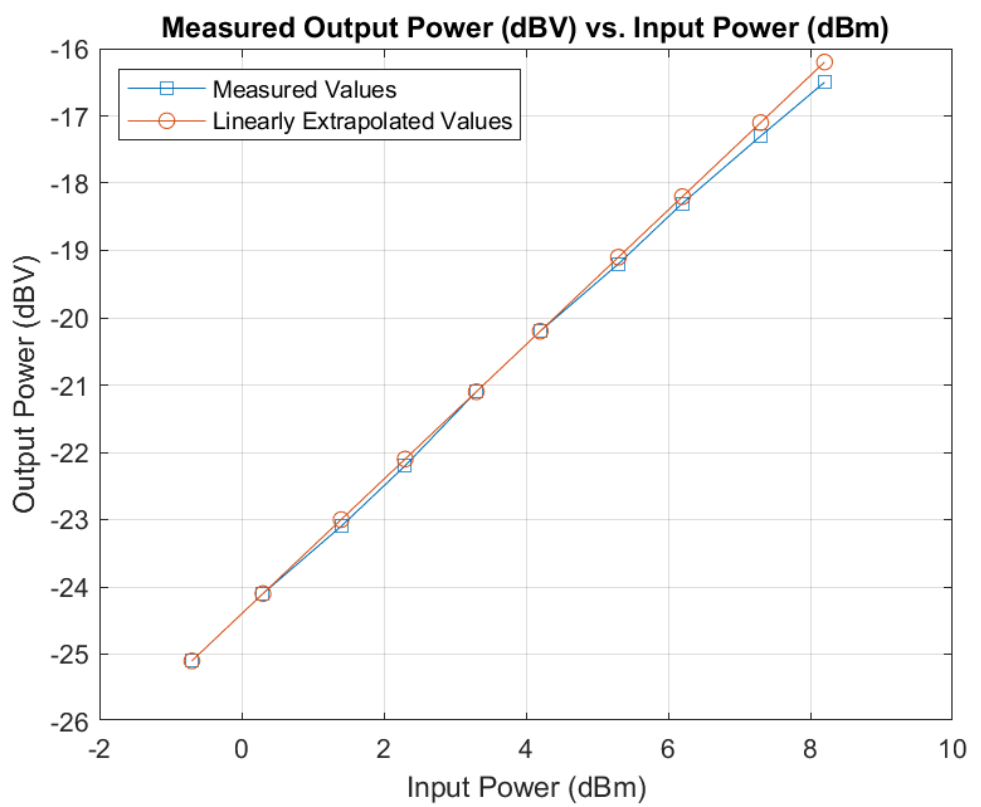

Figure 35: Beginning of Compression of IQ Mixer Output

\subsubsection{IQ Mixer Phase Characterization}

This subsection will cover the phase response characterization of the IQ mixer system in order to ensure there is minimal distortion in the output of the system. In order to accomplish this the circuit seen below in Figure 36 was used. The output of oscillator is split, attenuated, passed through a cable of varying length, and subsequently passed into the RF input of the IQ Mixer. 


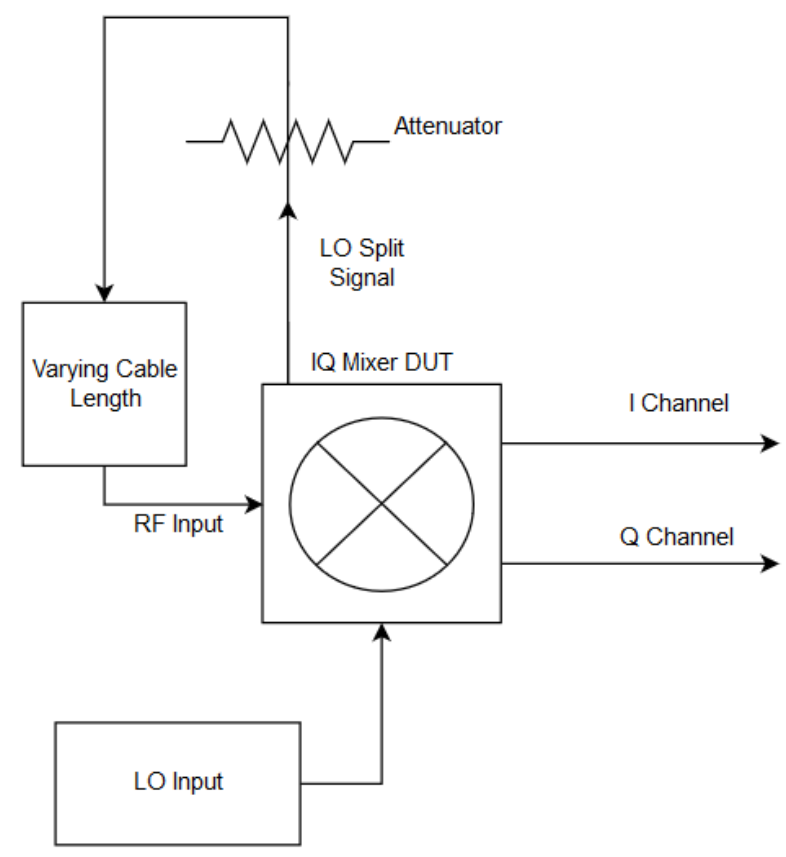

Figure 36: IQ Mixer Phase Response Characterization Schematic

Four different length SMA cables were implemented to measure the phase response of the system, before measuring the phase response of the system the electrical length of the four SMA cables were characterized using a Vector Network Analyzer. The cable number, physical length of the cable, measured electrical length of the cable, and the difference in effective electrical length compared to cable 1 can be seen below in Table 2, all of these measurements were performed using a frequency of $915 \mathrm{MHz}$.

Table 2: Electrical Lengths of Selected SMA Cables

\begin{tabular}{|c|c|c|c|}
\hline Cable & Physical Cable Length $(\mathrm{m})$ & Measured Electrical Length $\left(^{\circ}\right)$ & $\Delta$ Length $\left(^{\circ}\right)$ \\
\hline \hline 1 & 0.11 & 185.21 & 0 \\
\hline 2 & 0.15 & 244.62 & 59.41 \\
\hline 3 & 0.30 & 510.15 & -35.06 \\
\hline 4 & 0.61 & 963.17 & 57.96 \\
\hline
\end{tabular}


A $16 \mathrm{~dB}$ attenuator was added in series with each cable such so that the RF input power was $3.4 \mathrm{dBm}$, below the maximum linear input power value of $4.2 \mathrm{dBm}$, for the $11 \mathrm{~cm}$ cable, this signal can be seen below in Figure 37 .

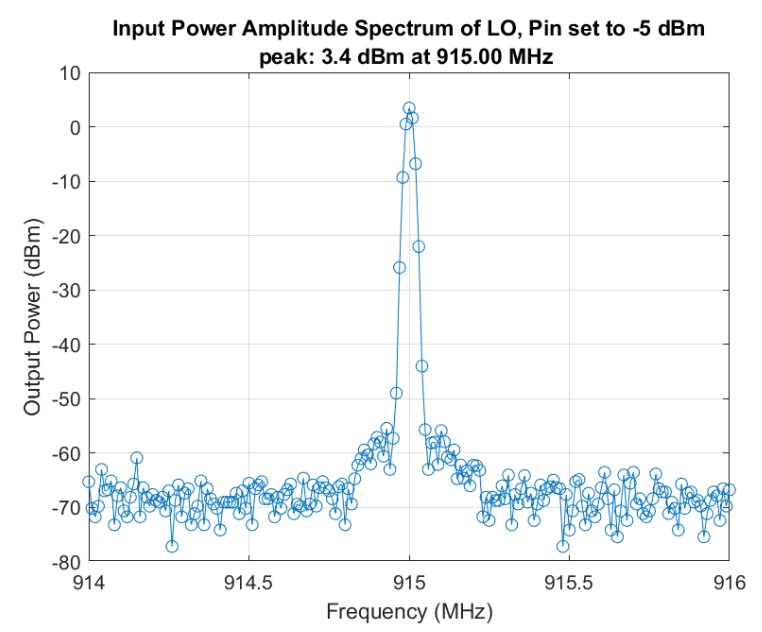

Figure 37: Input Signal for $11 \mathrm{~cm}$ SMA Cable With $16 \mathrm{~dB}$ Attenuator

Next these four cables were implemented and the output of the system was measured. Figures 38 through 45 show the data for each cable, as well the mean value of each of the sets of data.

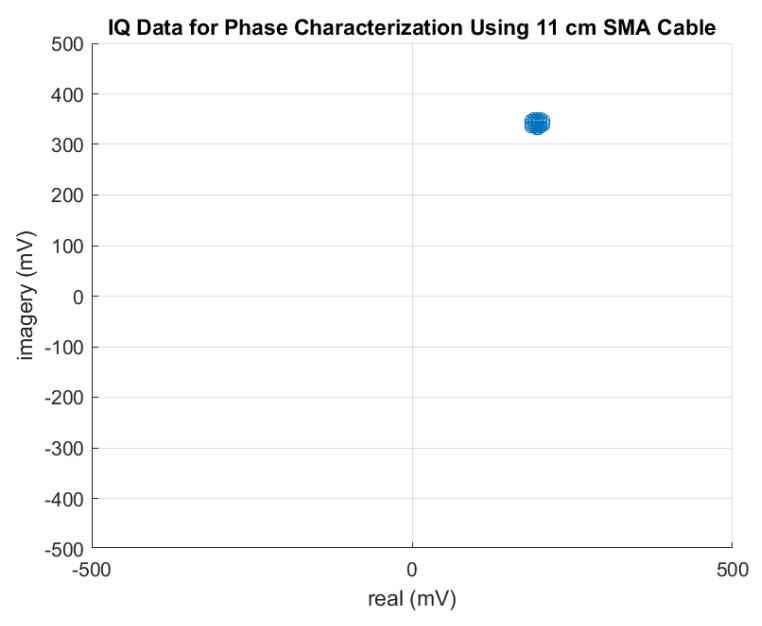

Figure 38: IQ Data Using 11cm SMA Cable 


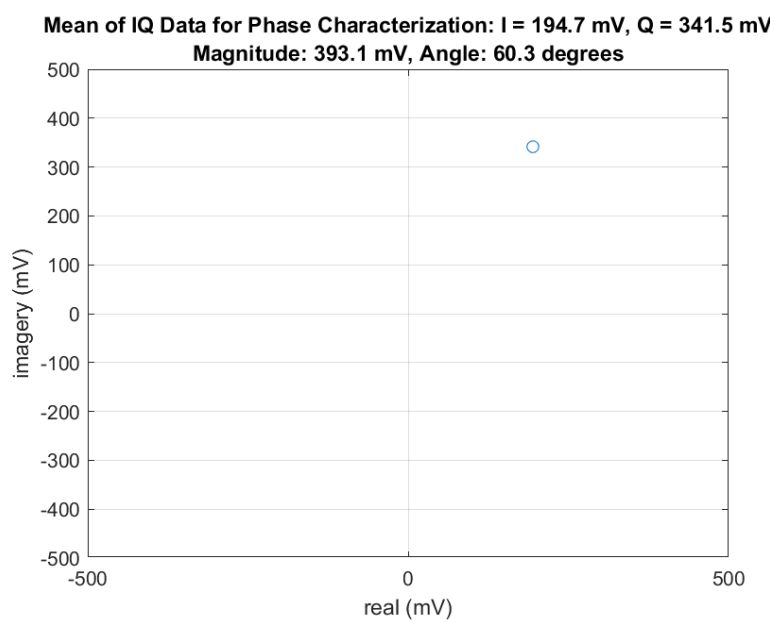

Figure 39: Mean of IQ Data Using 11cm SMA Cable

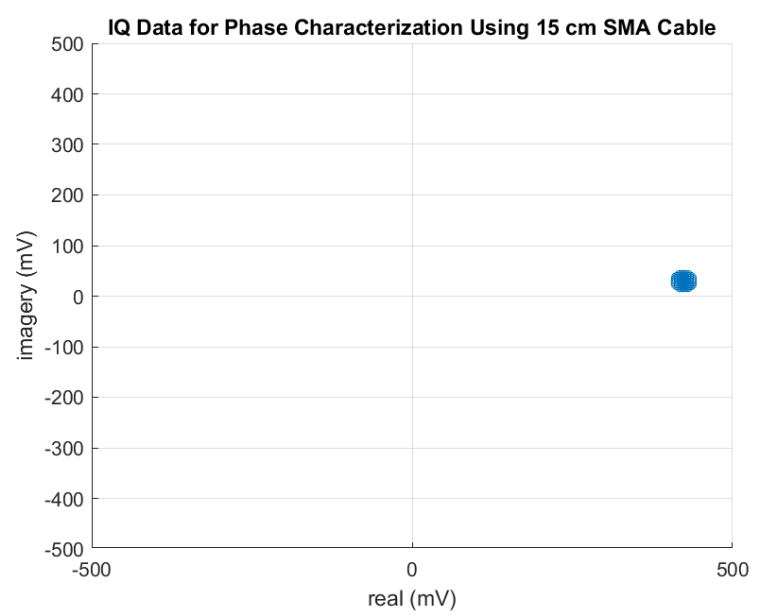

Figure 40: IQ Data Using 15cm SMA Cable 


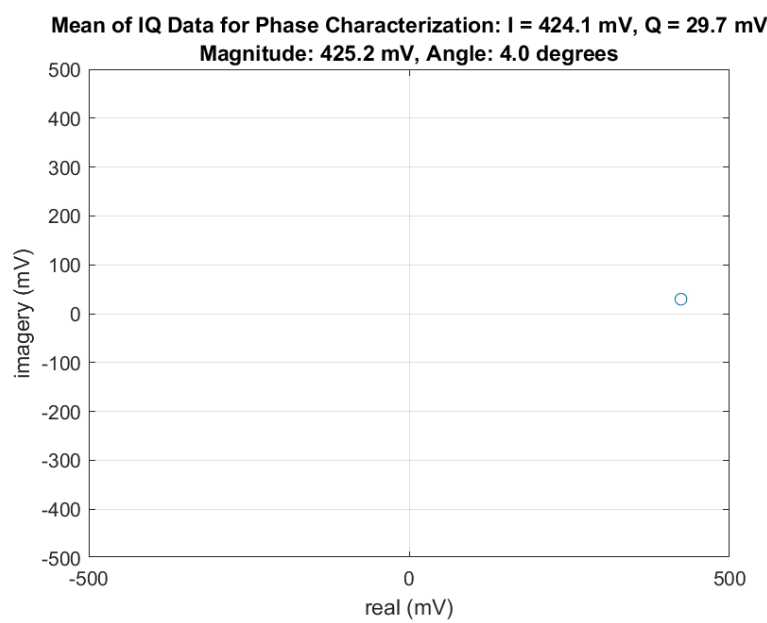

Figure 41: Mean of IQ Data Using 15cm SMA Cable

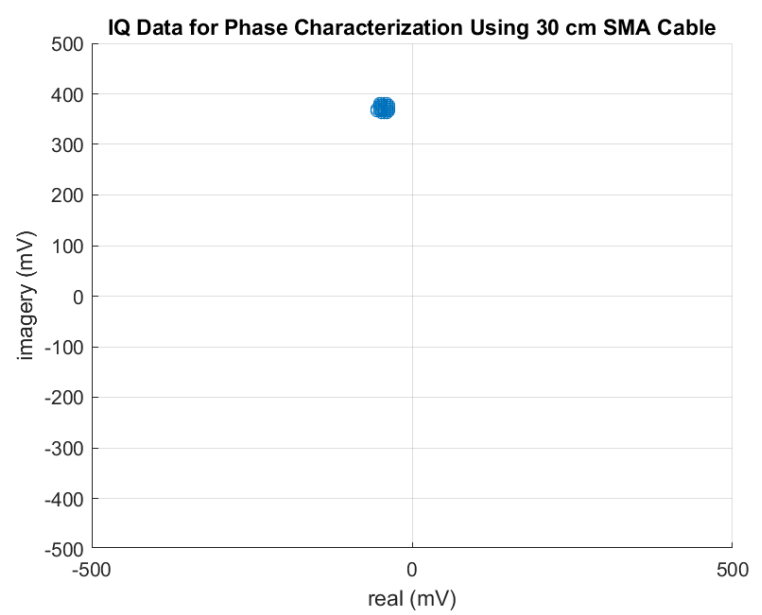

Figure 42: IQ Data Using 30cm SMA Cable 


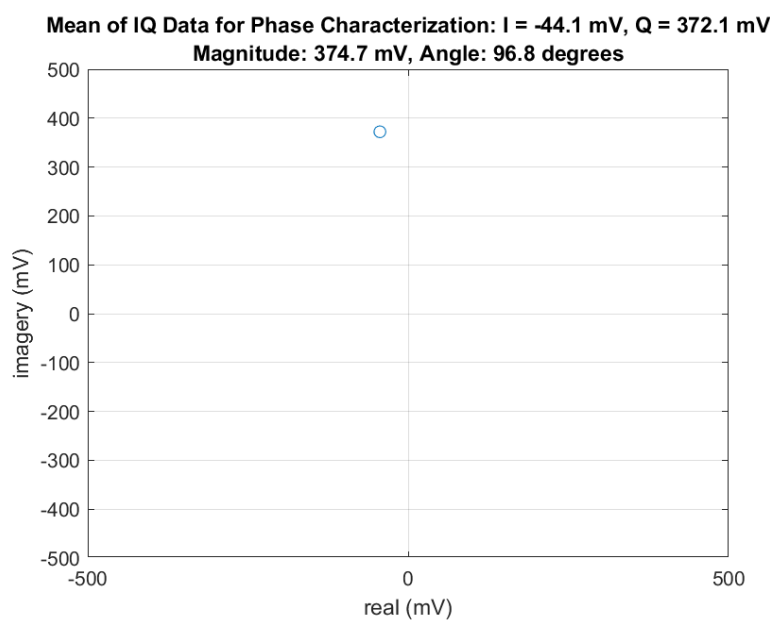

Figure 43: Mean of IQ Data Using 30cm SMA Cable

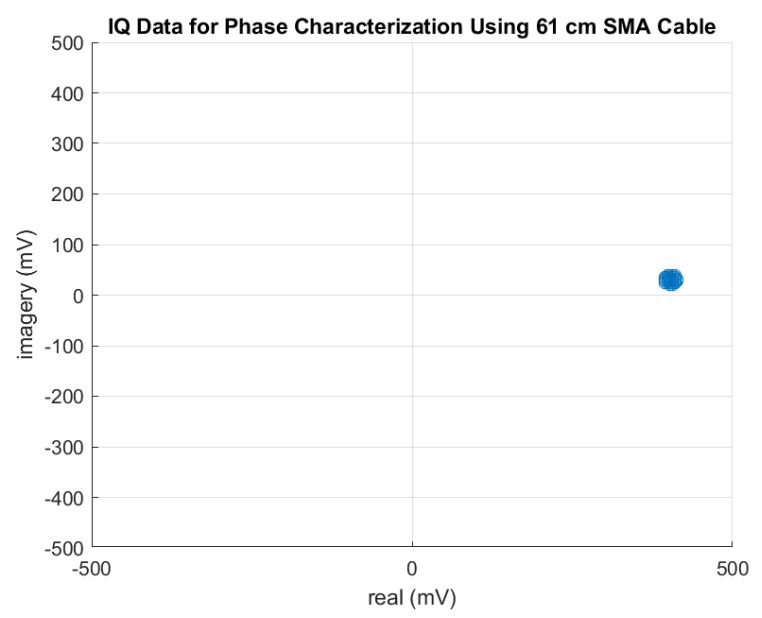

Figure 44: IQ Data Using 61cm SMA Cable 


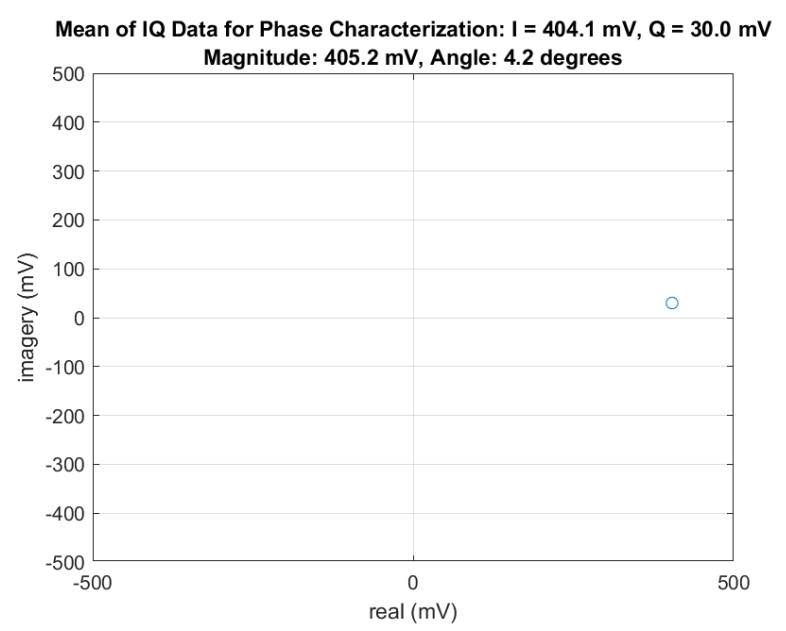

Figure 45: Mean of IQ Data Using 30cm SMA Cable

When comparing the output phase of the system cable 1 was used in order to set a reference for the output phase, cables 2 through 4 were then implemented and the output phase was measured. Using the difference in effective electrical length seen in Table 3 and the output phase using cable 1 an expected output phase for cables 2 through 4 was calculated. These values, the measured output phase response of the system for each cable, and the error between the expected and measured values can be seen below in Table 3 .

Table 3: Phase Response Measurement Results of IQ Mixer

\begin{tabular}{|c|c|c|c|}
\hline Cable & Expected Output Phase $\left(^{\circ}\right)$ & Measured Output Phase $\left(^{\circ}\right)$ & Error $\left(^{\circ}\right)$ \\
\hline \hline 1 & N/A & 60.3 & N/A \\
\hline 2 & 0.89 & 4.0 & 3.11 \\
\hline 3 & 95.36 & 96.8 & 1.44 \\
\hline 4 & 2.34 & 4.2 & 1.86 \\
\hline
\end{tabular}

From these measurements it can be seen the phase response of the system behaves nearly as expected. It can be seen that there is some error in the system being represented by a 
$1.44^{\circ}$ to $3.11^{\circ}$ error between the expected and measured results for cables 2 through 4 , this is likely due to IQ mismatch. Therefore these measurements were retaken while using the previously mentioned IQ correction. The results for these new measurements can be seen below in Figures 46 through 53 and Table 4.

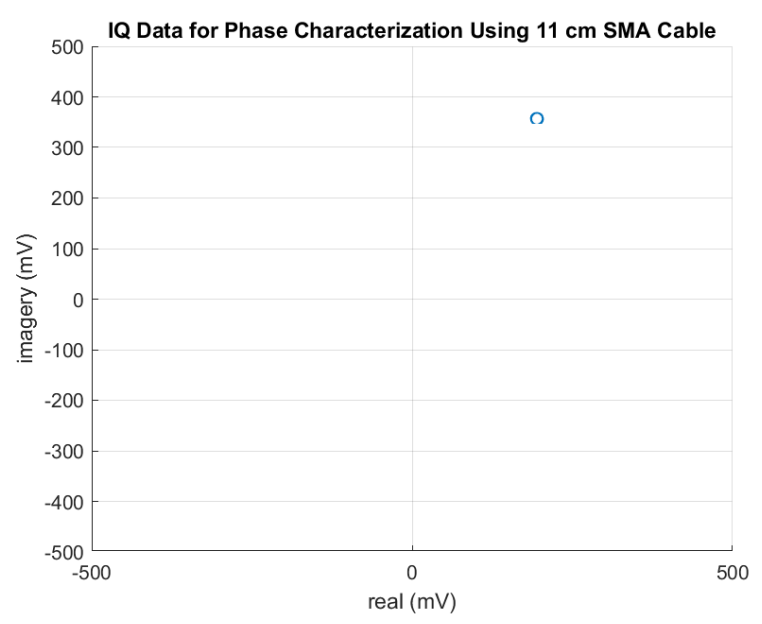

Figure 46: Corrected IQ Data Using 11cm SMA Cable

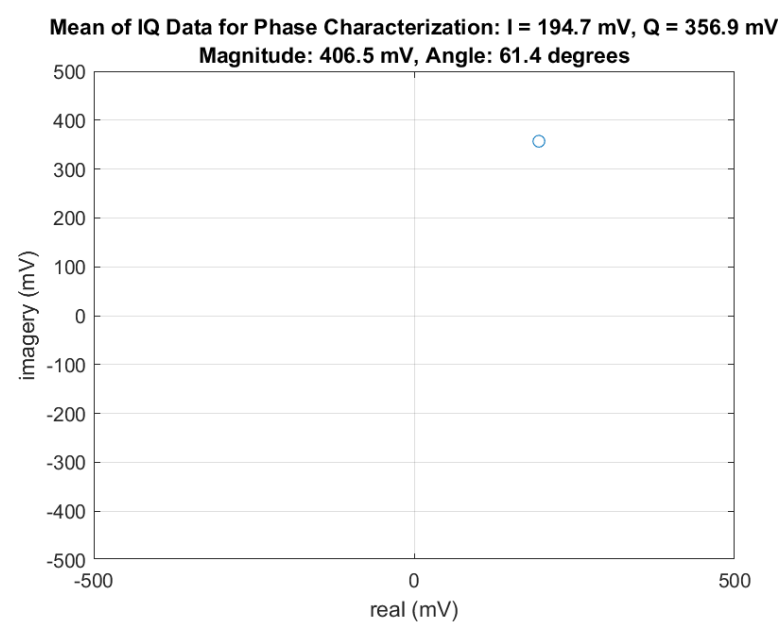

Figure 47: Corrected Mean of IQ Data Using 11cm SMA Cable 


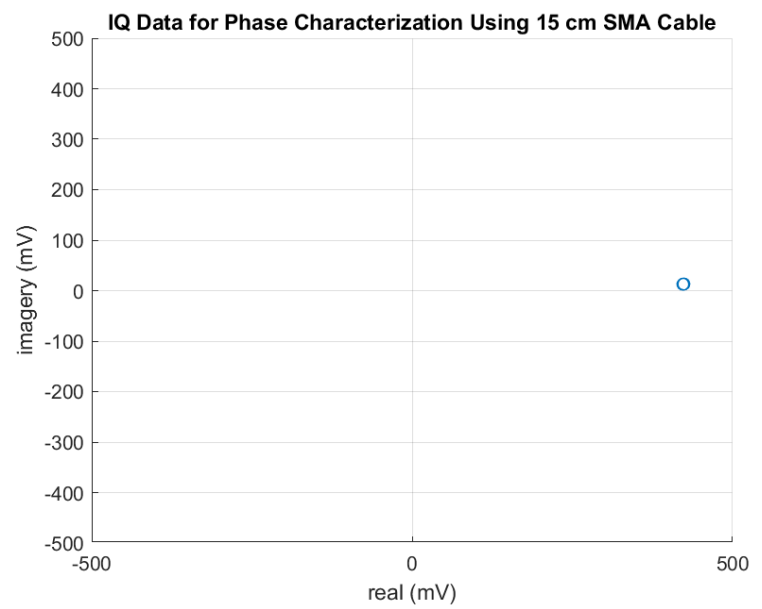

Figure 48: Corrected IQ Data Using 15cm SMA Cable

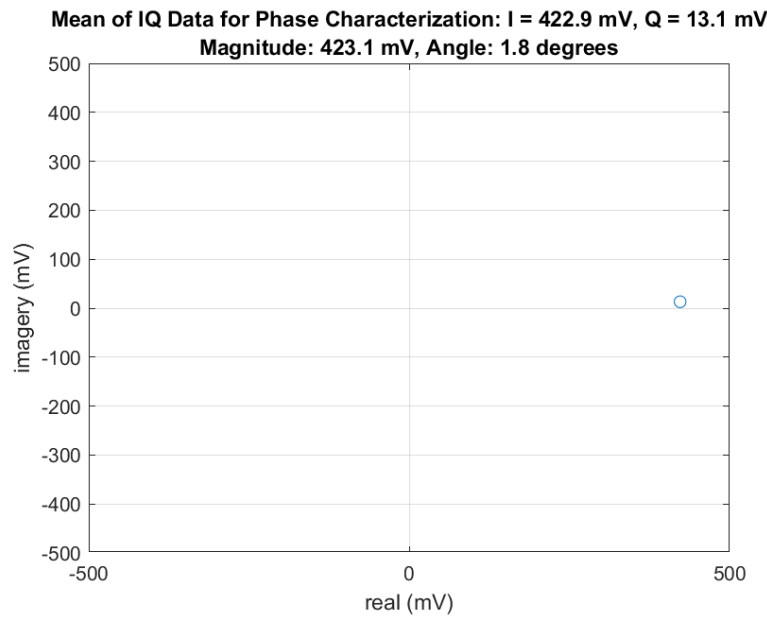

Figure 49: Corrected Mean of IQ Data Using 15cm SMA Cable 


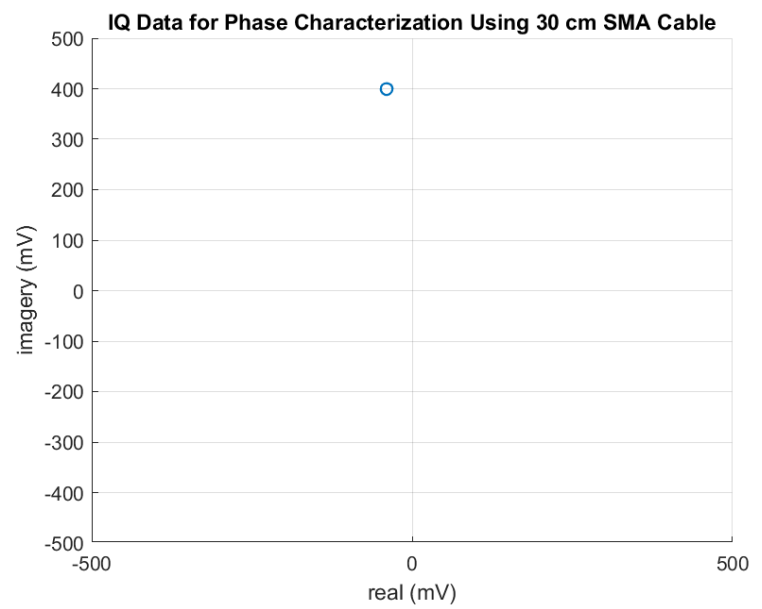

Figure 50: Corrected IQ Data Using 30cm SMA Cable

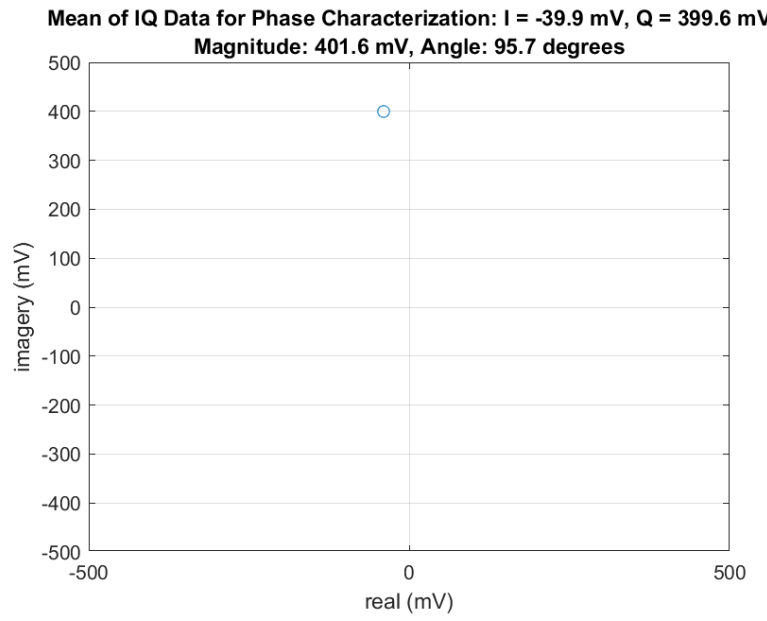

Figure 51: Corrected Mean of IQ Data Using 30cm SMA Cable 


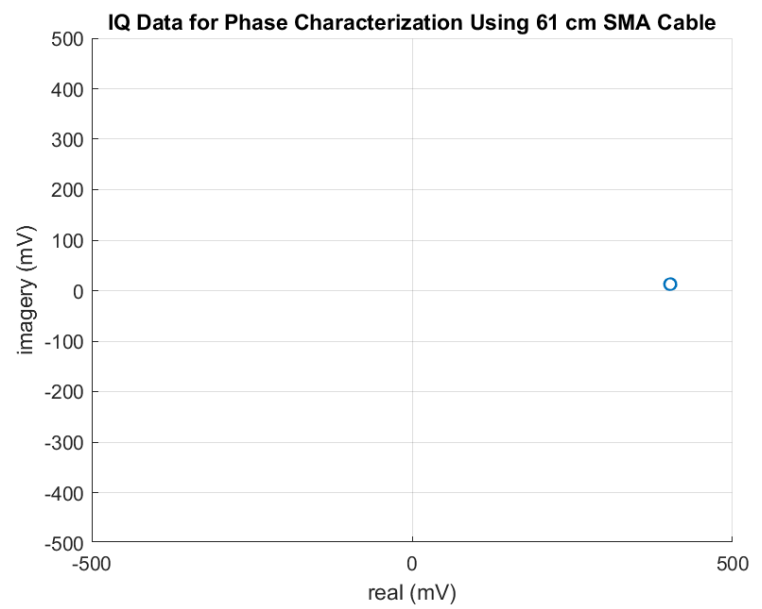

Figure 52: Corrected IQ Data Using 61cm SMA Cable

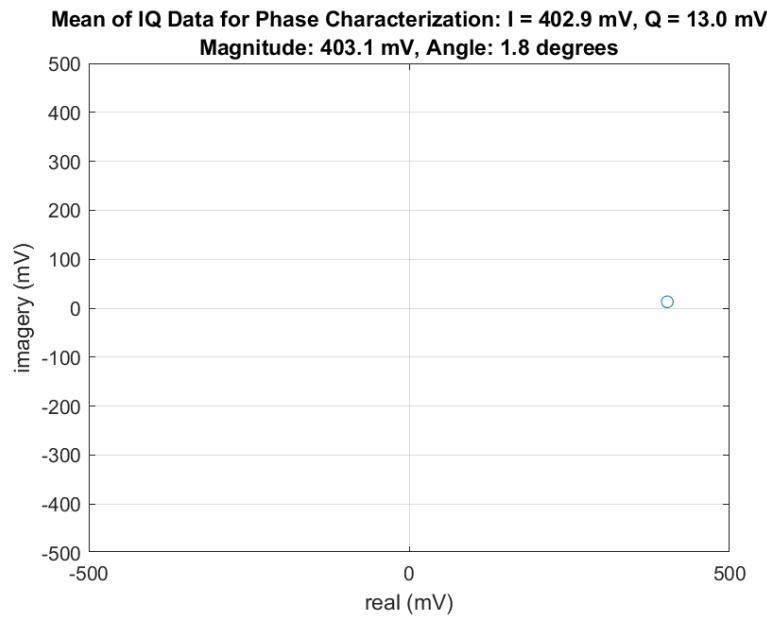

Figure 53: Corrected Mean of IQ Data Using 30cm SMA Cable 
Table 4: Corrected Phase Response Measurement Results of IQ Mixer

\begin{tabular}{|c|c|c|c|}
\hline Cable & Expected Phase Difference $\left({ }^{\circ}\right)$ & Measured Phase Difference $\left(^{\circ}\right)$ & Error $\left(^{\circ}\right)$ \\
\hline \hline 1 & N/A & 61.4 & N/A \\
\hline 2 & 1.99 & 1.8 & -0.19 \\
\hline 3 & 96.46 & 95.7 & -0.76 \\
\hline 4 & 3.44 & 1.8 & -1.64 \\
\hline
\end{tabular}

From these measurements it can be seen that the applied IQ correction reduces the IQ mismatch, therefore reducing the error between the expected and measured output phase to a new range of $0.19^{\circ}$ to $1.64^{\circ}$. The largest error is caused from the $61 \mathrm{~cm}$ cable, which compared to the previously largest error of $3.11^{\circ}$ of the $15 \mathrm{~cm}$ cable, is nearly half the magnitude.

\subsubsection{Synthesizer Phase Noise Characterization}

This subsection will cover characterization of the phase noise of the TPI synthesizer. As previously explained this thesis will use a modified version of the reference method for phase noise characterization. To this end the circuit seen below in Figure 54 will be used. In addition the PICOscope used will be set to AC couple mode, with a cutoff frequency of approximately $1 \mathrm{~Hz}$. 


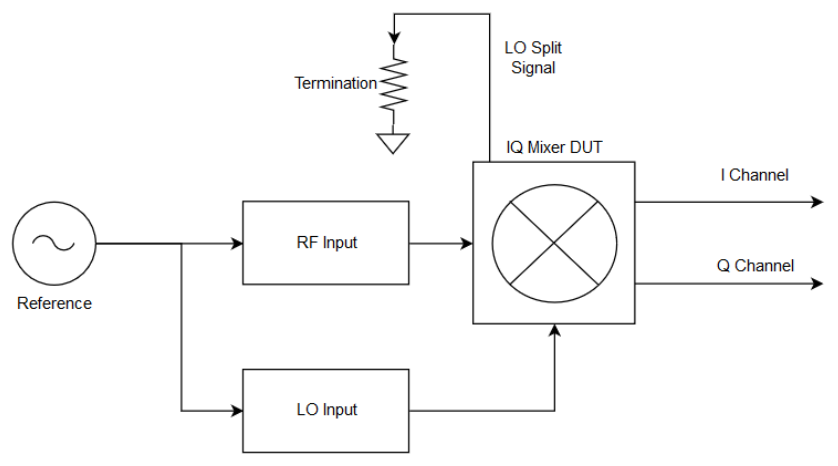

Figure 54: Synthesizer Phase Noise Characterization Schematic

The RF input was terminated and the output with just the LO tone being fed into the system was measured in order to determine the noise floor past which measurement would not be possible, the schematic used can be seen below in Figure 55 .

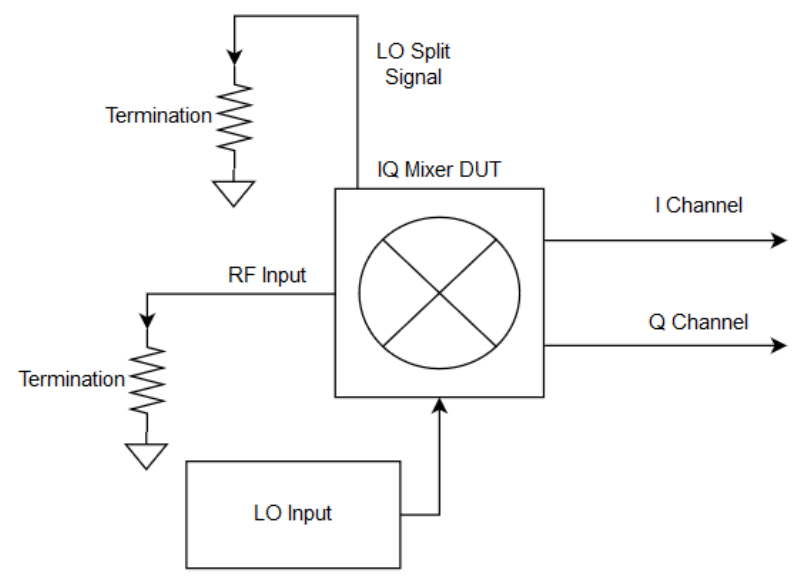

Figure 55: RF Input Terminated Schematic

The resulting spectrum can be seen below in Figures 56 and 57 . 


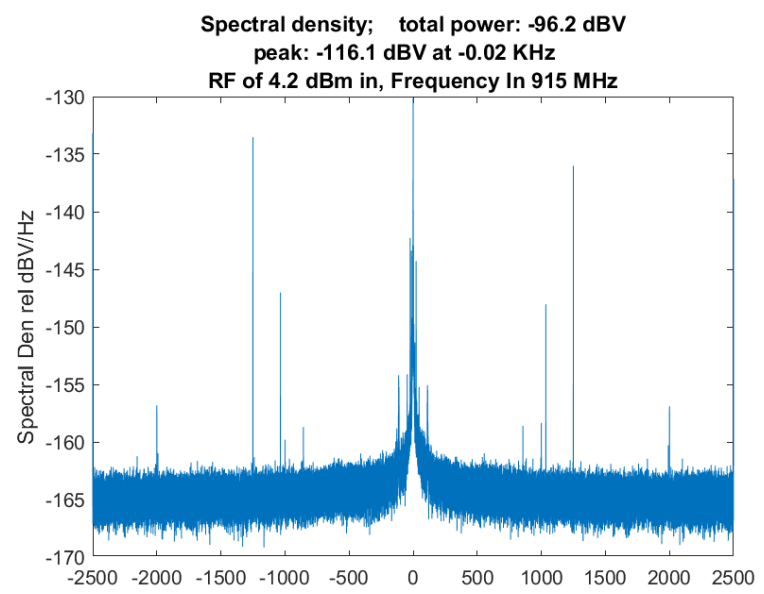

Figure 56: Measured Noise Floor With RF Input Terminated

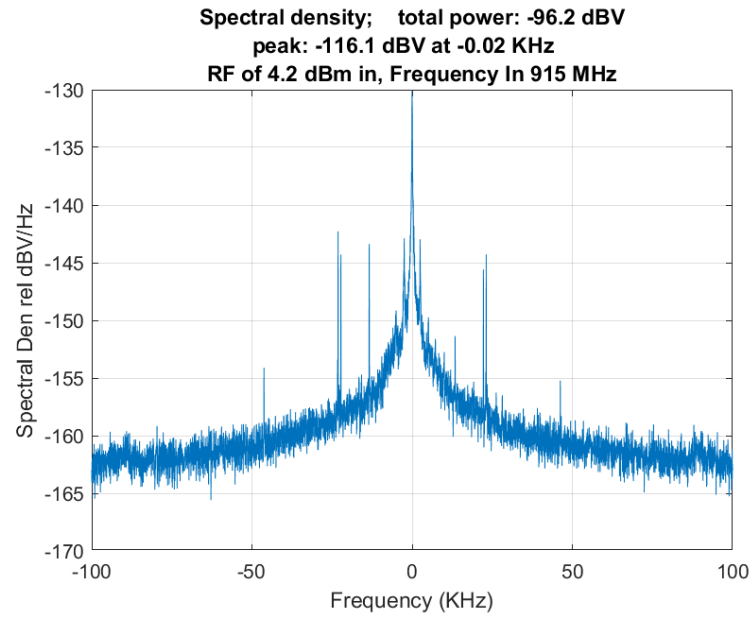

Figure 57: Measured Noise Floor With RF Input Terminated From -100 kHz to $100 \mathrm{kHz}$

From this spectrum the noise power was measured at the various frequencies of interest as seen below in Table 5 . 
Table 5: Noise Floor Spectral Density With RF Input Terminated

\begin{tabular}{|c|c|}
\hline Offset Frequency $(\mathrm{kHz})$ & Measured PN Spectral Density $(\mathrm{dBV} / \mathrm{Hz})$ \\
\hline \hline 20 & -157.9 \\
\hline 50 & -160.7 \\
\hline 100 & -162.1 \\
\hline 300 & -163.7 \\
\hline 1000 & -164.4 \\
\hline
\end{tabular}

The RF input power was set to $4.2 \mathrm{dBm}$ at the input of the IQ mixer, therefore using the previously characterized gain the carrier will mix to DC at an amplitude of $-20.2 \mathrm{~dB}$. The resulting spectral density for this measurement can be seen below in Figure 58 and Figure 59.

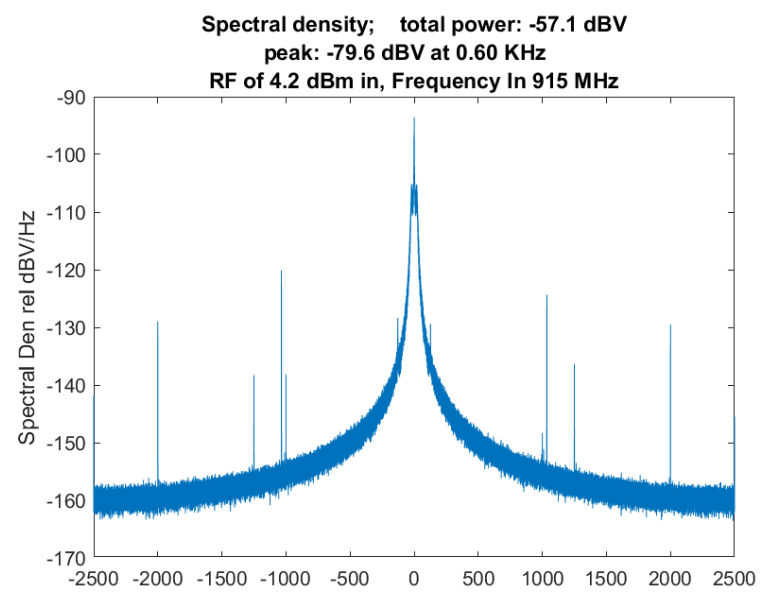

Figure 58: Phase Noise Spectral Density of Two Synthesizers 


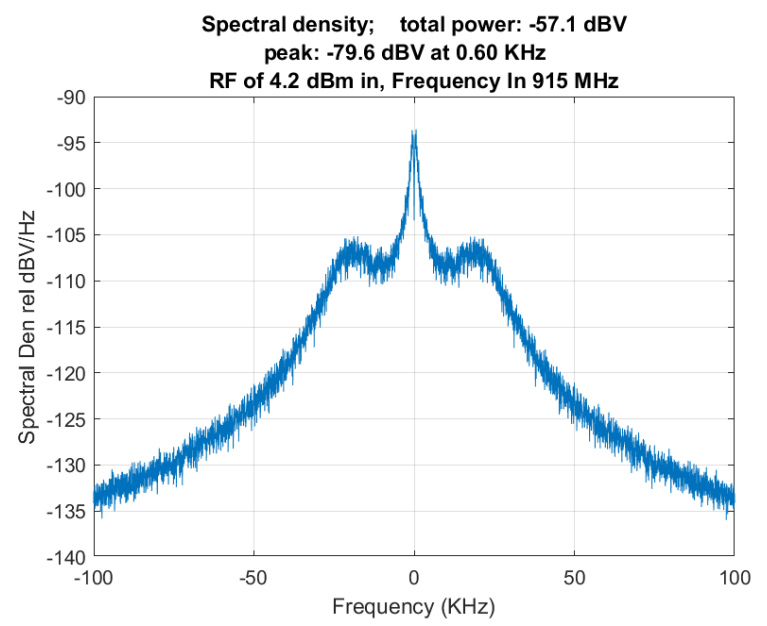

Figure 59: Phase Noise Spectral Density of Two Synthesizers From -100 kHz to $100 \mathrm{kHz}$

From these plots the noise floor was measured to be approximately $-159 \mathrm{dBV} / \mathrm{Hz}$, in order to determine the magnitude of the phase noise the linear equivalent power of the noise floor is subtracted from the measured value of phase noise. The measured phase noise spectral density at various frequencies, as well as the spectral density with the noise floor subtracted, can be seen below in Table 6 .

Table 6: Measured Phase Noise Spectral Density

\begin{tabular}{|c|c|c|}
\hline Offset Frequency $(\mathrm{kHz})$ & Measured PN Spectral Density $(\mathrm{dBV} / \mathrm{Hz})$ & PN Noise Floor Subtracted $(\mathrm{dBV} / \mathrm{Hz})$ \\
\hline \hline 20 & -107.0 & -107.0 \\
\hline 50 & -123.5 & -123.5 \\
\hline 100 & -133.4 & -133.4 \\
\hline 300 & -145.3 & -145.5 \\
\hline 1000 & -155.5 & -158.0 \\
\hline
\end{tabular}

Next $3 \mathrm{~dB}$ is subtracted from measured result due to the fact that two identical synthesizers are contributing to it. Lastly the signal is compared to the carrier power of $20.2 \mathrm{dBV}$ in order to determine the phase noise relative to the carrier this data can be seen below in Table 7 . 
Table 7: Phase Noise Spectral Density of TPI Synthesizer

\begin{tabular}{|c|c|c|}
\hline Offset Frequency $(\mathrm{kHz})$ & Single Synthesizer PN $(\mathrm{dBV} / \mathrm{Hz})$ & PN Relative to Carrier $(\mathrm{dBC} / \mathrm{Hz})$ \\
\hline \hline 20 & -110 & -89.8 \\
\hline 50 & -126.5 & -106.3 \\
\hline 100 & -136.4 & -116.2 \\
\hline 300 & -148.3 & -128.1 \\
\hline 1000 & -161.0 & -140.8 \\
\hline
\end{tabular}

\subsection{Phase Noise Cancellation Measurements}

This section will discuss the implementation of various systems in order to measure the phase noise cancellation and compare it to expected values. To this end the schematic seen below in Figure 60 will be used. The split LO signal within the IQ Mixer is put through the system under test and passed into the RF input of the IQ Mixer.

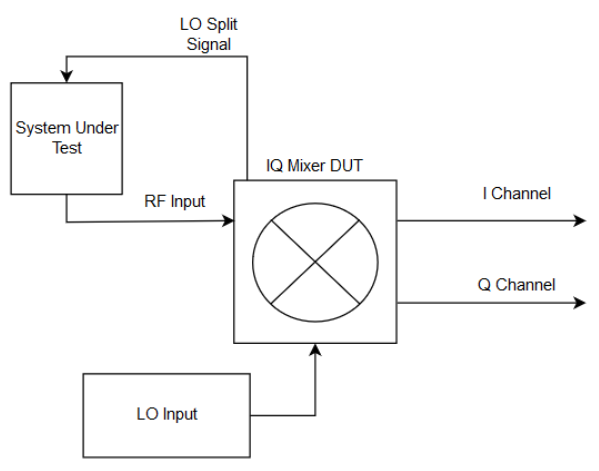

Figure 60: Synthesizer Phase Noise Cancellation Schematic

For the purposes of this thesis the system under test will consist of a number of RG-58 cable lengths, accompanied by a ZRL-1150LN+ amplifier and various attenuators. These attenuators and some of the cable were placed before the amplifier such that the input power would be $-20 \mathrm{dBm}$ or below. As the typical input referred $1 \mathrm{~dB}$ compression point of the 
ZRL-1150LN+ amplifier is provided as $-10 \mathrm{dBm}$ the input power was backed off a minimum of $10 \mathrm{~dB}$ in order to ensure linear functionality. However not all of the cable was placed prior to the amplifier in order to reduce the overall noise figure of the system. The general schematic for the system can be seen below in Figure 61 .

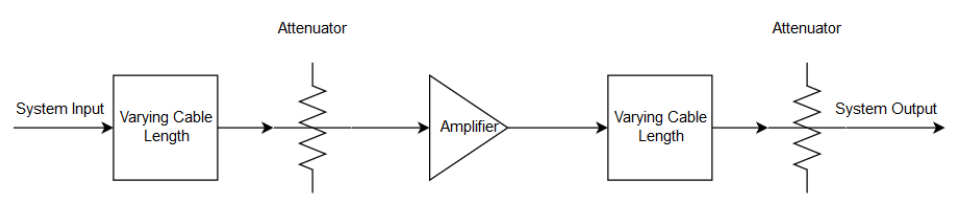

Figure 61: System Under Test Schematic

Additionally the input to the system under test, or the LO Split Signal, was measured by terminating the RF input as seen in the schematic below in Figure 62 .

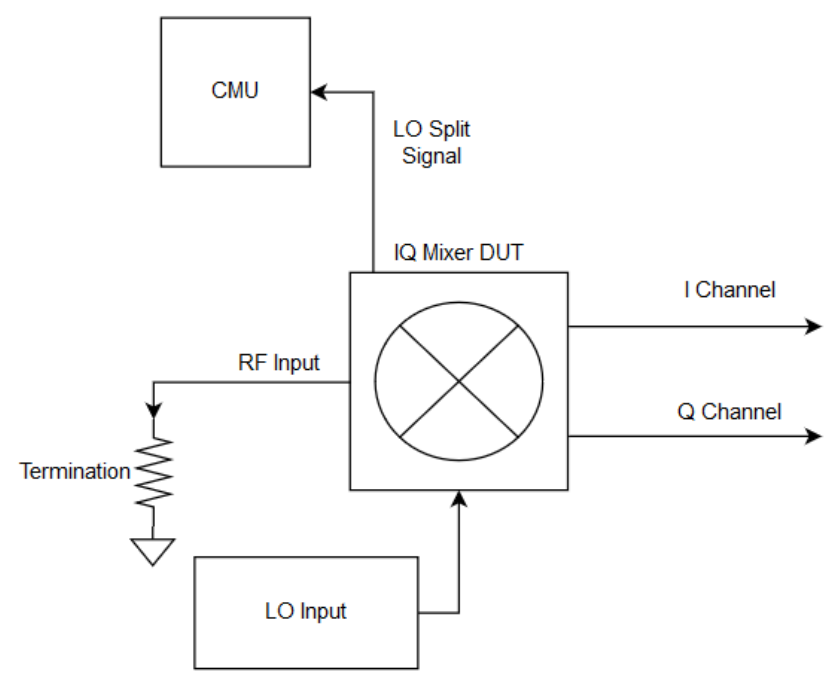

Figure 62: Splitter Output Power Schematic

The resulting spectrum can be seen below in Figure 63, the power of the signal was measured to be $20.0 \mathrm{dBm}$. 


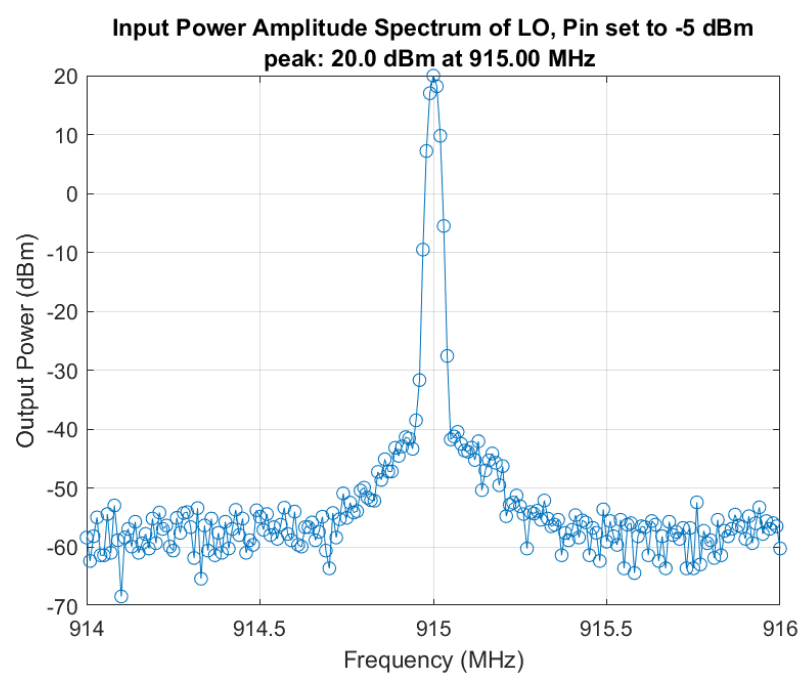

Figure 63: LO Split Signal Spectral Power 


\subsubsection{Phase Noise Cancellation of 90m SMA Cable System}

The first system consisted of 90m of RG-58 SMA Cable, a ZRL-1150LN+ amplifier, and an attenuator. The response of the system at various frequencies was measured using a Vector Network Analyzer (VNA) as seen below in Table 8, where $0 \mathrm{kHz}$ offset is $915 \mathrm{MHz}$.

Table 8: System Response Measurements Of 90m SMA Cable

\begin{tabular}{|c|c|c|}
\hline Offset Frequency $(\mathrm{kHz})$ & Amplitude Response $(\mathrm{dB})$ & Phase Response $\left(^{\circ}\right)$ \\
\hline \hline-1000 & -17.021 & 37.789 \\
\hline-300 & -17.103 & -71.406 \\
\hline-100 & -17.145 & -102.62 \\
\hline-50 & -17.142 & -110.41 \\
\hline-20 & -17.139 & -115.11 \\
\hline 0 & -17.137 & -118.25 \\
\hline 20 & -17.138 & -121.37 \\
\hline 50 & -17.147 & -126.05 \\
\hline 100 & -17.147 & -133.88 \\
\hline 300 & -17.184 & -165.14 \\
\hline 1000 & -17.276 & 88.557 \\
\hline
\end{tabular}

Using equation 57 and the system responses measured at each frequency offset the change in phase noise with respect to the carrier was calculated, the results for which can be seen below in Table 9. 
Table 9: Phase Noise Spectral Density of 90m system

\begin{tabular}{|c|c|}
\hline Offset Frequency $(\mathrm{kHz})$ & Calculated Change In Phase Noise $(\mathrm{dB})$ \\
\hline \hline 20 & -22.2 \\
\hline 50 & -14.3 \\
\hline 100 & -8.3 \\
\hline 300 & 1.0 \\
\hline 1000 & 8.8 \\
\hline
\end{tabular}

Next the system as implemented with the IQ Mixer, the input power to the RF port of the IQ mixer was measured using the schematic seen below in Figure 64 .

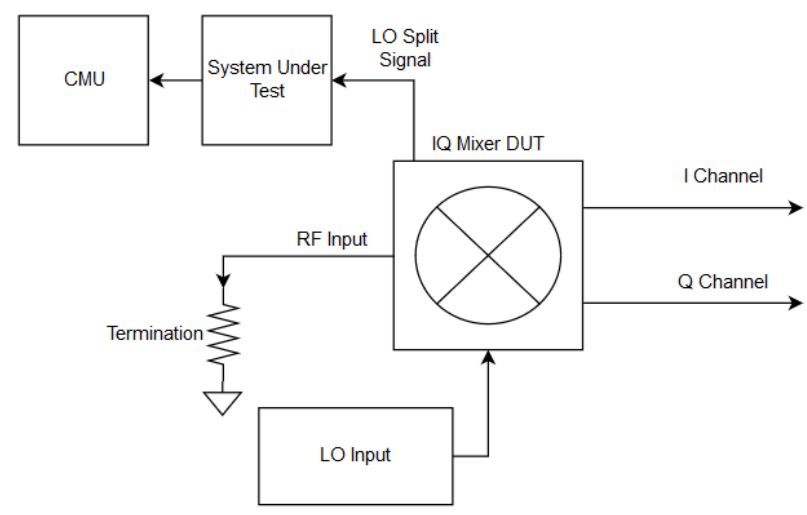

Figure 64: RF Input Power Schematic

The output power of the system was measured to be $3.4 \mathrm{dBm}$ as seen below in Figure 65 , in order to ensure it was below the maximum allowable value of $4.2 \mathrm{dBm}$. 


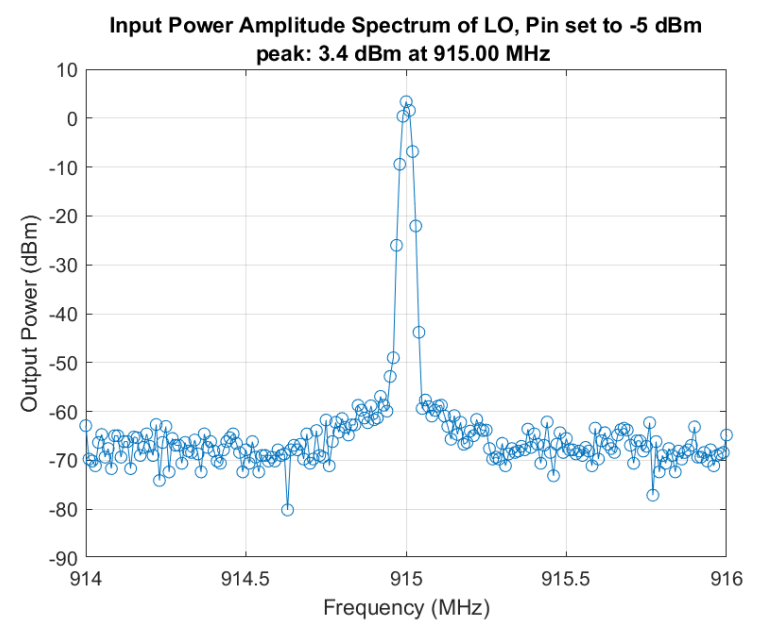

Figure 65: RF Input Power of 90m System

The system was then fully implemented with the IQ mixer, the resulting baseband phase noise can be seen below in Figure 66 and the zoomed in spectrum between $-100 \mathrm{kHz}$ and $100 \mathrm{kHz}$ can be seen in Figure 67.

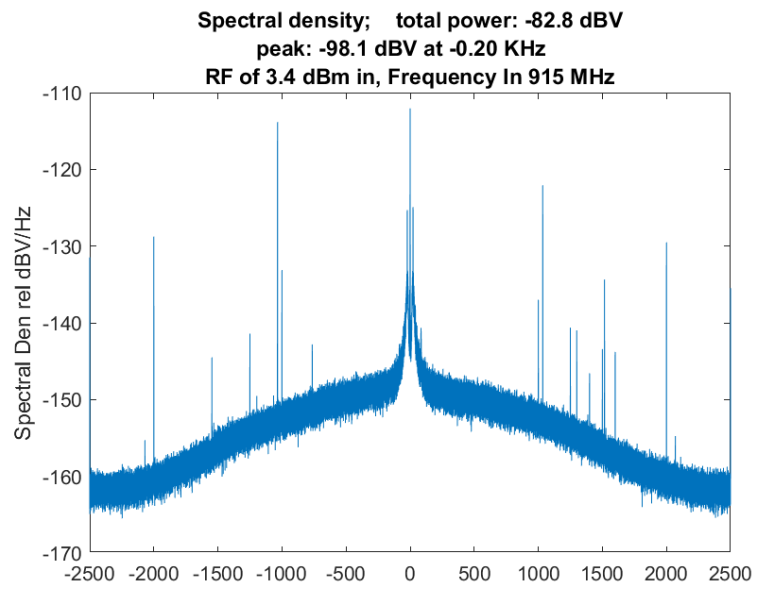

Figure 66: Phase Noise Spectral Density of 90m System 


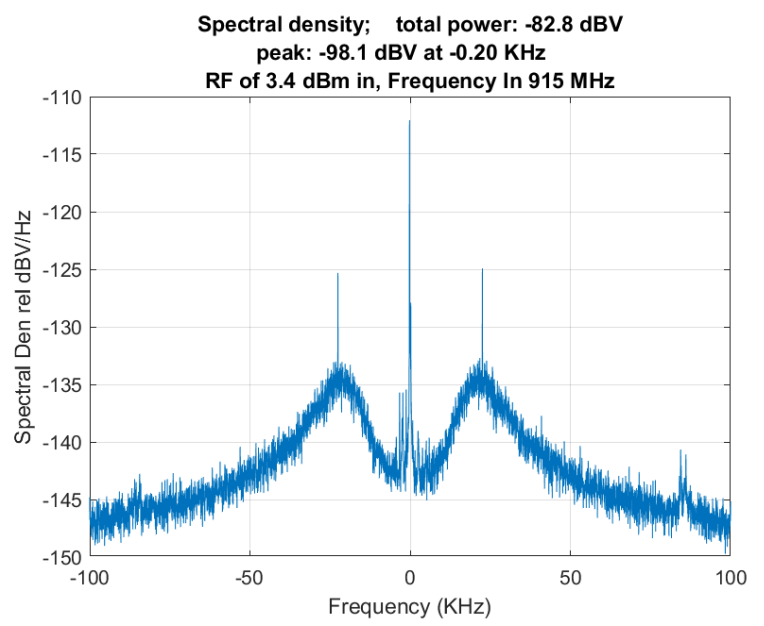

Figure 67: Phase Noise Spectral Density of 90m System From -100 kHz to $100 \mathrm{kHz}$

From this spectrum the new noise floor was measured to be $-161.4 \mathrm{dBV} / \mathrm{Hz}$, compared to the previous $164.4 \mathrm{dBV} / \mathrm{Hz}$. The added noise spectral density was calculated and added to the previously measured values of the noise floor in Table 5 , resulting in the new spectral noise density values seen below in Table 10 .

Table 10: 90m Cable Noise Floor Spectral Density With RF Input Terminated

\begin{tabular}{|c|c|}
\hline Offset Frequency $(\mathrm{kHz})$ & Calculated Noise Floor $(\mathrm{dBV} / \mathrm{Hz})$ \\
\hline \hline 20 & -157.0 \\
\hline 50 & -159.2 \\
\hline 100 & -160.1 \\
\hline 300 & -161.0 \\
\hline 1000 & -161.4 \\
\hline
\end{tabular}

From this spectrum the spectral density was measured at the same frequencies for which the phase noise was characterized, the resulting values can be seen below in Table 11. The linear equivalent power of the noise floor values in Table 10 were subtracted from each of 
the measured values and can also be seen in Table 11 .

Table 11: Phase Noise Of 90m SMA Cable

\begin{tabular}{|c|c|c|}
\hline Offset Frequency $(\mathrm{kHz})$ & Measured PN Spectral Density $(\mathrm{dBV} / \mathrm{Hz})$ & PN Spectral Density Subtracting Noise Floor $(\mathrm{dBV} / \mathrm{Hz})$ \\
\hline \hline 20 & -134.9 & -134.9 \\
\hline 50 & -142.6 & -142.7 \\
\hline 100 & -146.7 & -146.9 \\
\hline 300 & -149.2 & -149.5 \\
\hline 1000 & -152.6 & -153.2 \\
\hline
\end{tabular}

The carrier power of $-21 \mathrm{dBV}(3.4 \mathrm{dBV}-24.4 \mathrm{dBV} / \mathrm{dBm})$ was then subtracted from the newly calculated value accounting for the noise floor in order to calculated the phase noise relative to the carrier signal, the resulting values can be seen below in Table 12 .

Table 12: Phase Noise Spectral Density of 90m system

\begin{tabular}{|c|c|}
\hline Offset Frequency $(\mathrm{kHz})$ & PN Relative to Carrier $(\mathrm{dBC} / \mathrm{Hz})$ \\
\hline \hline 20 & -113.9 \\
\hline 50 & -121.7 \\
\hline 100 & -125.9 \\
\hline 300 & -128.5 \\
\hline 1000 & -132.2 \\
\hline
\end{tabular}

The phase noise of the TPI synthesizer was then subtracted from these measured phase noises in order to calculate the magnitude of the phase noise cancellation, the result can be seen below in Table 13. The error between the measured and calculated cancellation was calculated and is also shown below in Table 13 . 
Table 13: Cancellation Measurement for 90m Of Cable

\begin{tabular}{|c|c|c|c|}
\hline Offset Frequency $(\mathrm{kHz})$ & Measured Change In PN $(\mathrm{dB})$ & Calculated Change In PN $(\mathrm{dB})$ & Error $(\mathrm{dB})$ \\
\hline \hline 20 & -24.1 & -22.2 & -1.9 \\
\hline 50 & -15.4 & -14.3 & -1.1 \\
\hline 100 & -9.7 & -8.3 & -1.4 \\
\hline 300 & -0.4 & 1.0 & -1.4 \\
\hline 1000 & 8.6 & 8.8 & -0.2 \\
\hline
\end{tabular}




\subsubsection{Phase Noise Cancellation of 80m SMA Cable System}

The second system consisted of 80m of RG-58 SMA Cable, a ZRL-1150LN+ amplifier, and an attenuator. The response of the system at various frequencies was measured using a Vector Network Analyzer (VNA) as seen below in Table 14, where $0 \mathrm{kHz}$ offset is $915 \mathrm{MHz}$.

Table 14: System Response Measurements Of 80m SMA Cable

\begin{tabular}{|c|c|c|}
\hline Offset Frequency $(\mathrm{kHz})$ & Amplitude Response $(\mathrm{dB})$ & Phase Response $\left(^{\circ}\right)$ \\
\hline \hline-1000 & -16.661 & -76.807 \\
\hline-300 & -16.724 & -175.72 \\
\hline-100 & -16.754 & 155.98 \\
\hline-50 & -16.759 & 148.93 \\
\hline-20 & -16.761 & 155.71 \\
\hline 0 & -16.762 & 141.75 \\
\hline 20 & -16.763 & 139.08 \\
\hline 50 & -16.77 & 134.78 \\
\hline 100 & -16.767 & 126.67 \\
\hline 300 & -16.806 & 99.37 \\
\hline 1000 & -16.908 & 0.152 \\
\hline
\end{tabular}

Using equation 57, and the system responses measured at each frequency offset, the change in phase noise spectral density relative to the carrier was calculated. The results for which can be seen below in Table 15 . 
Table 15: Phase Noise Spectral Density of $80 \mathrm{~m}$ system

\begin{tabular}{|c|c|}
\hline Offset Frequency $(\mathrm{kHz})$ & Calculated Change In Phase Noise $(\mathrm{dB})$ \\
\hline \hline 20 & -23.15 \\
\hline 50 & -15.16 \\
\hline 100 & -9.12 \\
\hline 300 & 0.21 \\
\hline 1000 & 8.52 \\
\hline
\end{tabular}

Next the system as implemented with the IQ Mixer, the input power to the RF port of the IQ mixer was measured to be $3.5 \mathrm{dBm}$ as seen below in Figure 68, in order to ensure it was below the maximum allowable value of $4.2 \mathrm{dBm}$.

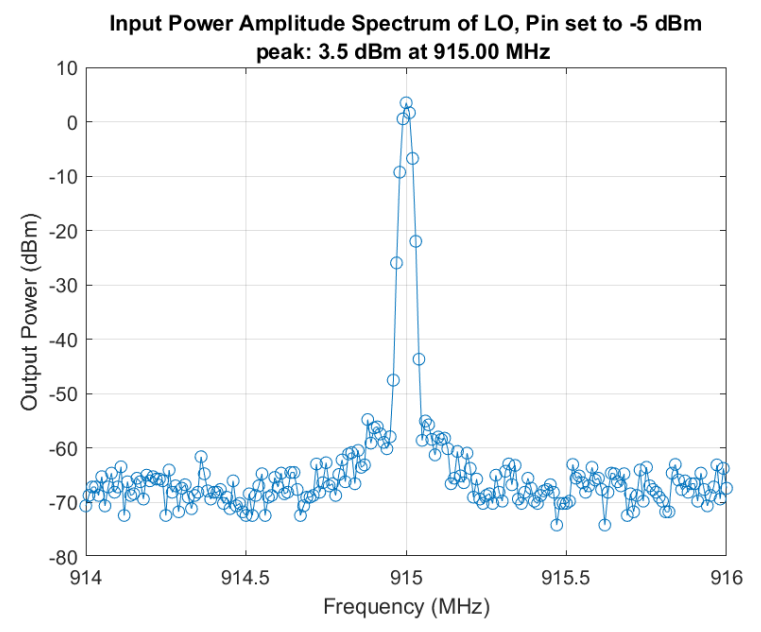

Figure 68: RF Input Power of 80m System

The system was then fully implemented with the IQ mixer, the resulting baseband phase noise can be seen below in Figure 69 and the zoomed in spectrum between $-100 \mathrm{kHz}$ and $100 \mathrm{kHz}$ can be seen in Figure 70 . 


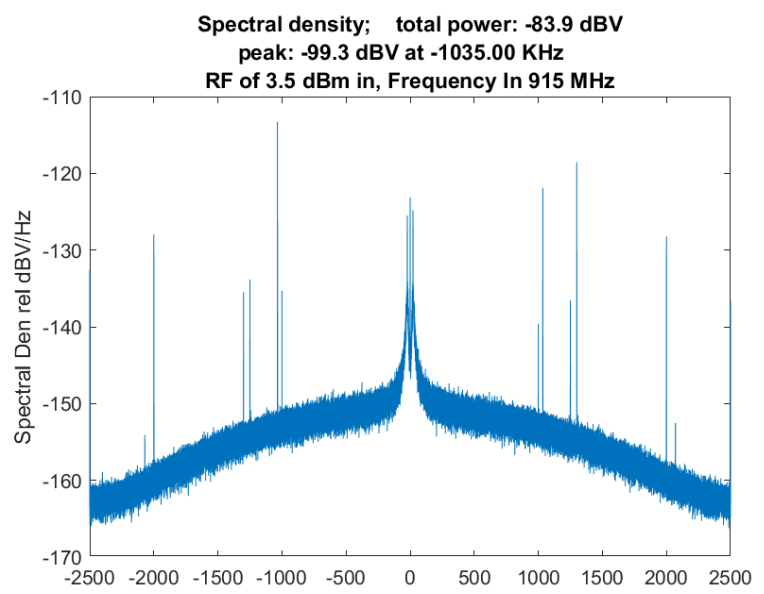

Figure 69: Phase Noise Spectral Density of 80m System

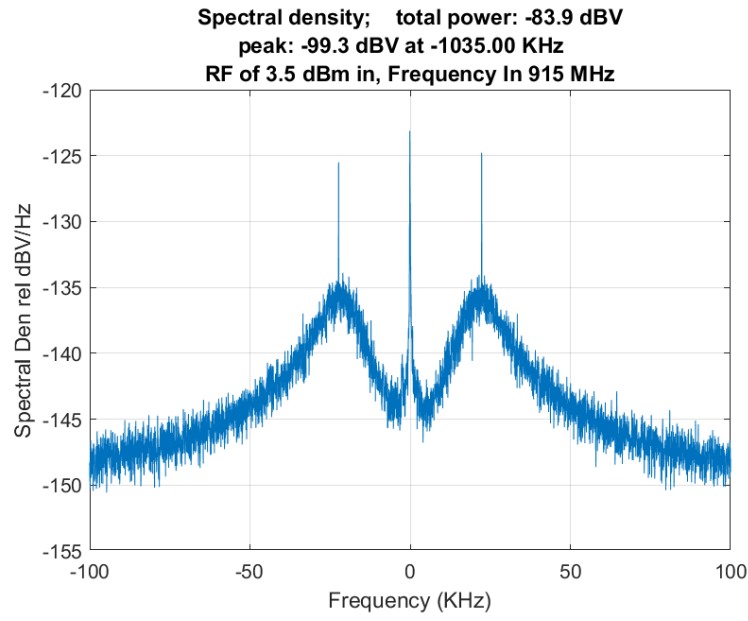

Figure 70: Phase Noise Spectral Density of 80m System From -100 kHz to $100 \mathrm{kHz}$

From this spectrum the new noise floor was measured to be $-161.4 \mathrm{dBV} / \mathrm{Hz}$, compared to the previous $164.4 \mathrm{dBV} / \mathrm{Hz}$. The added noise spectral density was calculated and added to the previously measured values of the noise floor in Table 5 , resulting in the new spectral noise density values seen below in Table 16 . 
Table 16: 80m Cable Noise Floor Spectral Density With RF Input Terminated

\begin{tabular}{|c|c|}
\hline Offset Frequency $(\mathrm{kHz})$ & Calculated Noise Floor $(\mathrm{dBV} / \mathrm{Hz})$ \\
\hline \hline 20 & -157.0 \\
\hline 50 & -159.2 \\
\hline 100 & -160.1 \\
\hline 300 & -161.0 \\
\hline 1000 & -161.4 \\
\hline
\end{tabular}

From this spectrum the spectral density was measured at the same frequencies for which the phase noise was characterized, the resulting values can be seen below in Table 17 . The linear equivalent power of the noise floor values in Table 16 were subtracted from each of the measured values and can also be seen in Table 17 .

Table 17: Phase Noise Of 80m SMA Cable

\begin{tabular}{|c|c|c|}
\hline Offset Frequency $(\mathrm{kHz})$ & Measured PN Spectral Density $(\mathrm{dBV} / \mathrm{Hz})$ & PN Spectral Density Subtracting Noise Floor $(\mathrm{dBV} / \mathrm{Hz})$ \\
\hline \hline 20 & -135.5 & -135.5 \\
\hline 50 & -144.0 & -144.1 \\
\hline 100 & -148.0 & -148.3 \\
\hline 300 & -150.4 & -150.8 \\
\hline 1000 & -152.8 & -153.4 \\
\hline
\end{tabular}

The carrier power of $-20.9 \mathrm{dBV}(3.5 \mathrm{dBV}-24.4 \mathrm{dBV} / \mathrm{dBm})$ was then subtracted from the newly calculated value accounting for the noise floor in order to calculated the phase noise relative to the carrier signal, the resulting values can be seen below in Table 18 . 
Table 18: Phase Noise Spectral Density of $80 \mathrm{~m}$ system

\begin{tabular}{|c|c|}
\hline Offset Frequency $(\mathrm{kHz})$ & PN Relative to Carrier $(\mathrm{dBC} / \mathrm{Hz})$ \\
\hline \hline 20 & -114.6 \\
\hline 50 & -123.2 \\
\hline 100 & -127.4 \\
\hline 300 & -129.9 \\
\hline 1000 & -132.5 \\
\hline
\end{tabular}

The phase noise of the TPI synthesizer was then subtracted from these measured phase noises in order to calculate the magnitude of the phase noise cancellation, the result can be seen below in Table 19. The error between the measured and calculated cancellation was calculated and is also shown below in Table 19.

Table 19: Cancellation Measurement for $80 \mathrm{~m}$ Of Cable

\begin{tabular}{|c|c|c|c|}
\hline Offset Frequency $(\mathrm{kHz})$ & Measured Change In PN $(\mathrm{dB})$ & Calculated Change In PN $(\mathrm{dB})$ & Error $(\mathrm{dB})$ \\
\hline \hline 20 & -24.8 & -23.11 & -1.7 \\
\hline 50 & -16.9 & -15.2 & -1.7 \\
\hline 100 & -11.2 & -9.1 & -2.1 \\
\hline 300 & -1.8 & 0.2 & -2.0 \\
\hline 1000 & 8.3 & 8.5 & -0.2 \\
\hline
\end{tabular}




\subsubsection{Phase Noise Cancellation of 70m SMA Cable System}

The third system consisted of $70 \mathrm{~m}$ of RG-58 SMA Cable, a ZRL-1150LN+ amplifier, and an attenuator. The response of the system at various frequencies was measured using a Vector Network Analyzer (VNA) as seen below in Table 20, where $0 \mathrm{kHz}$ offset is $915 \mathrm{MHz}$.

Table 20: System Response Measurements Of 70m SMA Cable

\begin{tabular}{|c|c|c|}
\hline Offset Frequency $(\mathrm{kHz})$ & Amplitude Response $(\mathrm{dB})$ & Phase Response $\left(^{\circ}\right)$ \\
\hline \hline-1000 & -16.821 & -114.73 \\
\hline-300 & -16.773 & 155.95 \\
\hline-100 & -16.784 & 130.71 \\
\hline-50 & -16.785 & 124.57 \\
\hline-20 & -16.786 & 120.89 \\
\hline 0 & -16.786 & 117.52 \\
\hline 20 & -16.789 & 115.9 \\
\hline 50 & -16.792 & 111.98 \\
\hline 100 & -16.782 & 105.47 \\
\hline 300 & -16.837 & 79.982 \\
\hline 1000 & -17.034 & -9.18 \\
\hline
\end{tabular}

Using equation 57, and the system responses measured at each frequency offset, the change in phase noise spectral density relative to the carrier was calculated. The results for which can be seen below in Table 21 . 
Table 21: Phase Noise Spectral Density of 70m system

\begin{tabular}{|c|c|}
\hline Offset Frequency $(\mathrm{kHz})$ & Calculated Change In Phase Noise $(\mathrm{dB})$ \\
\hline \hline 20 & -23.7 \\
\hline 50 & -16.1 \\
\hline 100 & -10.1 \\
\hline 300 & -0.7 \\
\hline 1000 & 8.0 \\
\hline
\end{tabular}

Next the system as implemented with the IQ Mixer, the input power to the RF port of the IQ mixer was measured to be $3.5 \mathrm{dBm}$ as seen below in Figure 71 , in order to ensure it was below the maximum allowable value of $4.2 \mathrm{dBm}$.

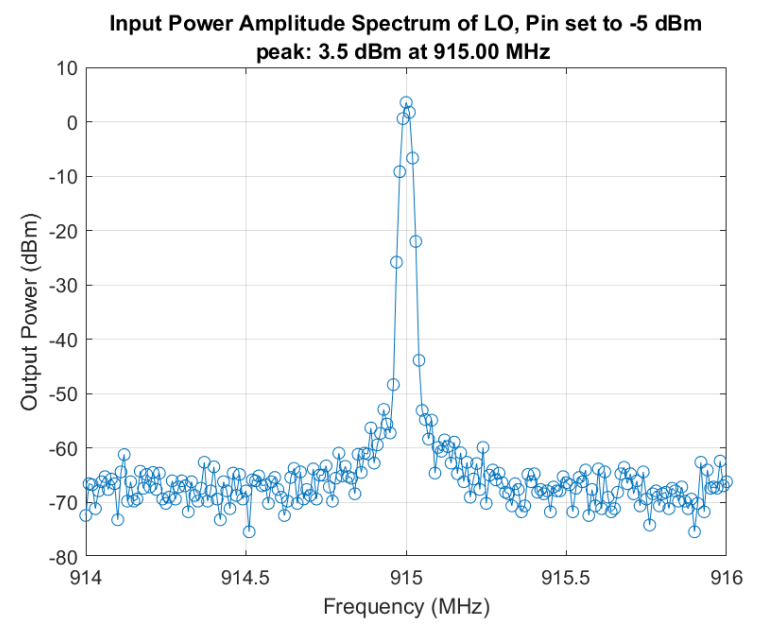

Figure 71: RF Input Power of 70m System

The system was then fully implemented with the IQ mixer, the resulting baseband phase noise can be seen below in Figure 72 and the zoomed in spectrum between $-100 \mathrm{kHz}$ and $100 \mathrm{kHz}$ can be seen in Figure 73 . 


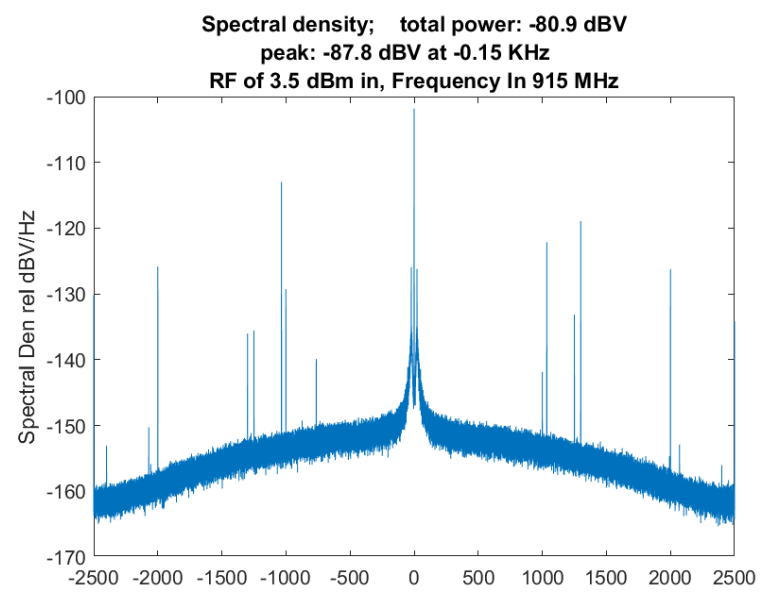

Figure 72: Phase Noise Spectral Density of 70m System

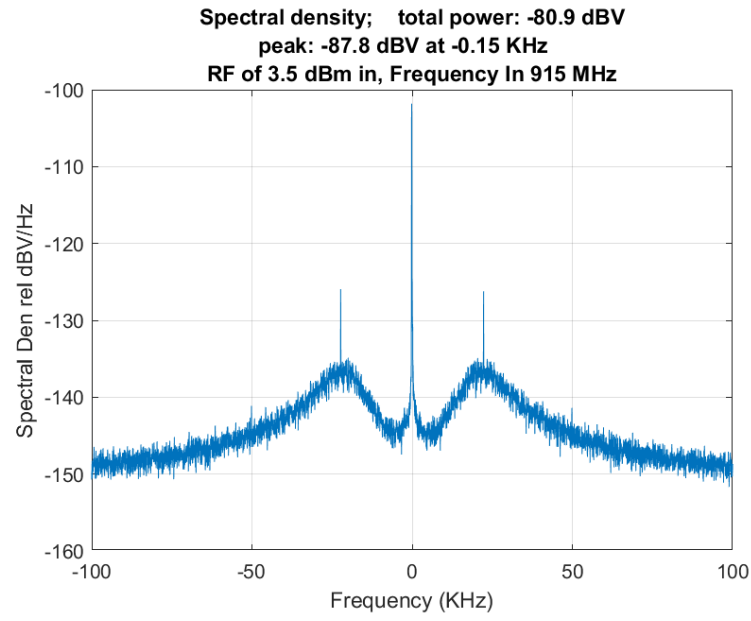

Figure 73: Phase Noise Spectral Density of 70m System From -100 kHz to $100 \mathrm{kHz}$

From this spectrum the new noise floor was measured to be $-161.4 \mathrm{dBV} / \mathrm{Hz}$, compared to the previous $164.4 \mathrm{dBV} / \mathrm{Hz}$. The added noise spectral density was calculated and added to the previously measured values of the noise floor in Table 5 , resulting in the new spectral noise density values seen below in Table 22 . 
Table 22: 70m Cable Noise Floor Spectral Density With RF Input Terminated

\begin{tabular}{|c|c|}
\hline Offset Frequency $(\mathrm{kHz})$ & Calculated Noise Floor $(\mathrm{dBV} / \mathrm{Hz})$ \\
\hline \hline 20 & -157.0 \\
\hline 50 & -159.2 \\
\hline 100 & -160.1 \\
\hline 300 & -161.0 \\
\hline 1000 & -161.4 \\
\hline
\end{tabular}

From this spectrum the spectral density was measured at the same frequencies for which the phase noise was characterized, the resulting values can be seen below in Table 23 . The linear equivalent power of the noise floor values in Table 22 were subtracted from each of the measured values and can also be seen in Table 23 .

Table 23: Phase Noise Of 70m SMA Cable

\begin{tabular}{|c|c|c|}
\hline Offset Frequency $(\mathrm{kHz})$ & Measured PN Spectral Density $(\mathrm{dBV} / \mathrm{Hz})$ & PN Spectral Density Subtracting Noise Floor $(\mathrm{dBV} / \mathrm{Hz})$ \\
\hline \hline 20 & -136.5 & -136.5 \\
\hline 50 & -145.1 & -145.3 \\
\hline 100 & -148.9 & -149.2 \\
\hline 300 & -151.3 & -151.8 \\
\hline 1000 & -153.4 & -154.1 \\
\hline
\end{tabular}

The carrier power of $-20.9 \mathrm{dBV}(3.5 \mathrm{dBV}-24.4 \mathrm{dBV} / \mathrm{dBm})$ was then subtracted from the newly calculated value accounting for the noise floor in order to calculated the phase noise relative to the carrier signal, the resulting values can be seen below in Table 24 . 
Table 24: Phase Noise Spectral Density of 70m system

\begin{tabular}{|c|c|}
\hline Offset Frequency $(\mathrm{kHz})$ & PN Relative to Carrier $(\mathrm{dBC} / \mathrm{Hz})$ \\
\hline \hline 20 & -115.6 \\
\hline 50 & -124.4 \\
\hline 100 & -128.3 \\
\hline 300 & -130.9 \\
\hline 1000 & -133.2 \\
\hline
\end{tabular}

The phase noise of the TPI synthesizer was then subtracted from these measured phase noises in order to calculate the magnitude of the phase noise cancellation, the result can be seen below in Table 25. The error between the measured and calculated cancellation was calculated and is also shown below in Table 25 .

Table 25: Cancellation Measurement for 70m Of Cable

\begin{tabular}{|c|c|c|c|}
\hline Offset Frequency $(\mathrm{kHz})$ & Measured Change In PN $(\mathrm{dB})$ & Calculated Change In PN $(\mathrm{dB})$ & Error $(\mathrm{dB})$ \\
\hline \hline 20 & -25.8 & -23.7 & -2.1 \\
\hline 50 & -18.1 & -16.1 & -2.0 \\
\hline 100 & -12.1 & -10.1 & -2.0 \\
\hline 300 & -2.8 & -0.7 & -2.1 \\
\hline 1000 & 7.6 & 8.0 & -0.4 \\
\hline
\end{tabular}




\subsubsection{Phase Noise Cancellation of 60m SMA Cable System}

The fourth system consisted of $60 \mathrm{~m}$ of RG-58 SMA Cable, a ZRL-1150LN+ amplifier, and an attenuator. The response of the system at various frequencies was measured using a Vector Network Analyzer (VNA) as seen below in Table 26, where $0 \mathrm{kHz}$ offset is $915 \mathrm{MHz}$.

Table 26: System Response Measurements Of 60m SMA Cable

\begin{tabular}{|c|c|c|}
\hline Offset Frequency $(\mathrm{kHz})$ & Amplitude Response $(\mathrm{dB})$ & Phase Response $\left(^{\circ}\right)$ \\
\hline \hline-1000 & -16.267 & 50.276 \\
\hline-300 & -16.308 & -26.645 \\
\hline-100 & -16.318 & -48.526 \\
\hline-50 & -16.319 & -53.966 \\
\hline-20 & -16.321 & -57.263 \\
\hline 0 & -16.319 & -59.587 \\
\hline 20 & -16.321 & -61.636 \\
\hline 50 & -16.326 & -64.942 \\
\hline 100 & -16.329 & -70.443 \\
\hline 300 & -16.375 & -92.373 \\
\hline 1000 & -16.466 & -169.18 \\
\hline
\end{tabular}

Using equation 57, and the system responses measured at each frequency offset, the change in phase noise spectral density relative to the carrier was calculated. The results for which can be seen below in Table 27 . 
Table 27: Phase Noise Spectral Density of $60 \mathrm{~m}$ system

\begin{tabular}{|c|c|}
\hline Offset Frequency $(\mathrm{kHz})$ & Calculated Change In Phase Noise $(\mathrm{dB})$ \\
\hline \hline 20 & -25.24 \\
\hline 50 & -17.37 \\
\hline 100 & -11.37 \\
\hline 300 & -1.95 \\
\hline 1000 & 7.26 \\
\hline
\end{tabular}

Next the system as implemented with the IQ Mixer, the input power to the RF port of the IQ mixer was measured to be $4.1 \mathrm{dBm}$ as seen below in Figure 74, in order to ensure it was below the maximum allowable value of $4.2 \mathrm{dBm}$.

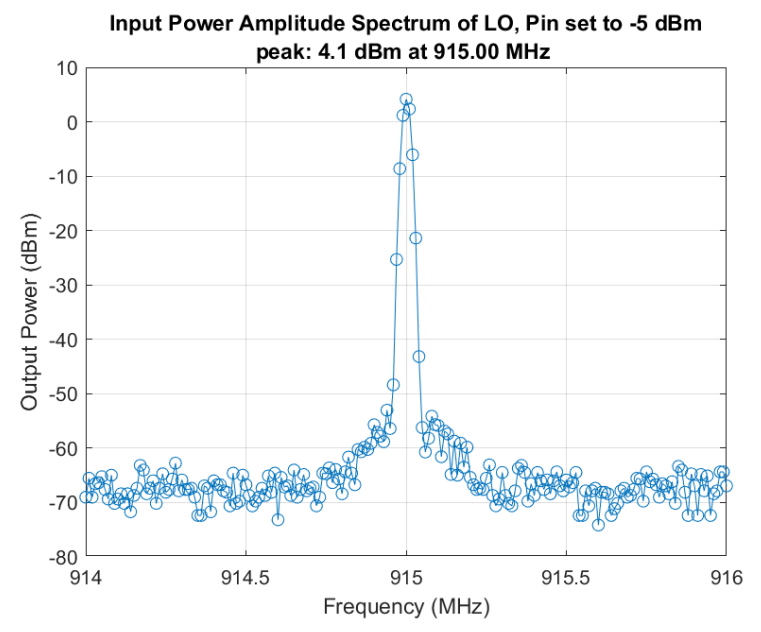

Figure 74: RF Input Power of 60m System

The system was then fully implemented with the IQ mixer, the resulting baseband phase noise can be seen below in Figure 75 and the zoomed in spectrum between $-100 \mathrm{kHz}$ and $100 \mathrm{kHz}$ can be seen in Figure 76 . 


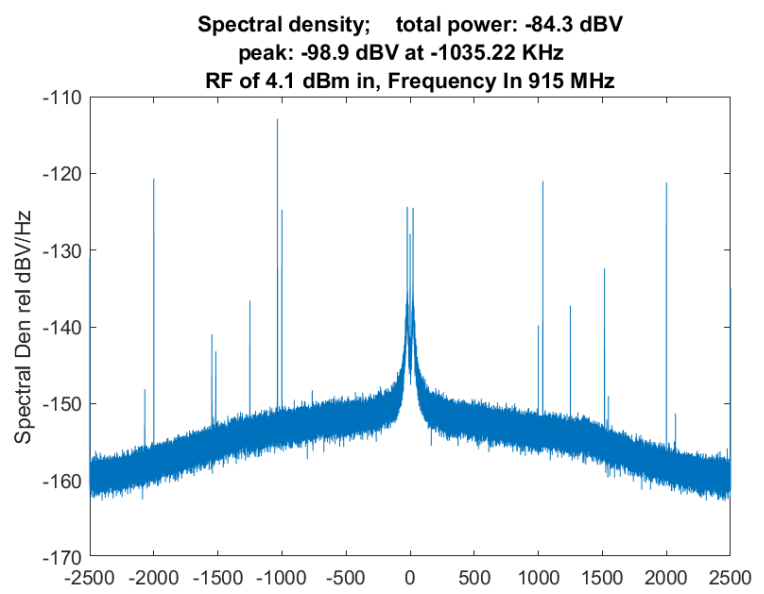

Figure 75: Phase Noise Spectral Density of $60 \mathrm{~m}$ System

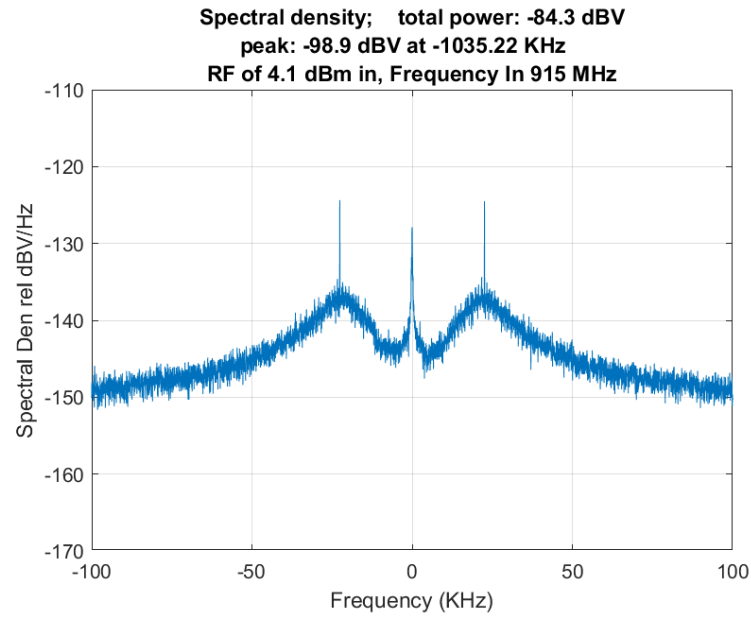

Figure 76: Phase Noise Spectral Density of 60m System From -100 kHz to $100 \mathrm{kHz}$

From this spectrum the new noise floor was measured to be $-159.3 \mathrm{dBV} / \mathrm{Hz}$, compared to the previous $164.4 \mathrm{dBV} / \mathrm{Hz}$. The added noise spectral density was calculated and added to the previously measured values of the noise floor in Table 5 , resulting in the new spectral noise density values seen below in Table 28 . 
Table 28: 60m Cable Noise Floor Spectral Density With RF Input Terminated

\begin{tabular}{|c|c|}
\hline Offset Frequency $(\mathrm{kHz})$ & Calculated Noise Floor $(\mathrm{dBV} / \mathrm{Hz})$ \\
\hline \hline 20 & -156.1 \\
\hline 50 & -157.8 \\
\hline 100 & -158.5 \\
\hline 300 & -159.1 \\
\hline 1000 & -159.3 \\
\hline
\end{tabular}

From this spectrum the spectral density was measured at the same frequencies for which the phase noise was characterized, the resulting values can be seen below in Table 29. The linear equivalent power of the noise floor values in Table 28 were subtracted from each of the measured values and can also be seen in Table 29 .

Table 29: Phase Noise Of 60m SMA Cable

\begin{tabular}{|c|c|c|}
\hline Offset Frequency $(\mathrm{kHz})$ & Measured PN Spectral Density $(\mathrm{dBV} / \mathrm{Hz})$ & PN Spectral Density Subtracting Noise Floor $(\mathrm{dBV} / \mathrm{Hz})$ \\
\hline \hline 20 & -136.7 & -136.8 \\
\hline 50 & -145.4 & -145.8 \\
\hline 100 & -149.3 & -149.9 \\
\hline 300 & -151.8 & -152.7 \\
\hline 1000 & -153.6 & -155.0 \\
\hline
\end{tabular}

The carrier power of $-20.3 \mathrm{dBV}(4.1 \mathrm{dBV}-24.4 \mathrm{dBV} / \mathrm{dBm})$ was then subtracted from the newly calculated value accounting for the noise floor in order to calculated the phase noise relative to the carrier signal, the resulting values can be seen below in Table 30 . 
Table 30: Phase Noise Spectral Density of $60 \mathrm{~m}$ system

\begin{tabular}{|c|c|}
\hline Offset Frequency $(\mathrm{kHz})$ & PN Relative to Carrier $(\mathrm{dBC} / \mathrm{Hz})$ \\
\hline \hline 20 & -116.5 \\
\hline 50 & -125.5 \\
\hline 100 & -129.6 \\
\hline 300 & -132.4 \\
\hline 1000 & -132.7 \\
\hline
\end{tabular}

The phase noise of the TPI synthesizer was then subtracted from these measured phase noises in order to calculate the magnitude of the phase noise cancellation, the result can be seen below in Table 31. The error between the measured and calculated cancellation was calculated and is also shown below in Table 31.

Table 31: Cancellation Measurement for 60m Of Cable

\begin{tabular}{|c|c|c|c|}
\hline Offset Frequency $(\mathrm{kHz})$ & Measured Change In PN $(\mathrm{dB})$ & Calculated Change In PN $(\mathrm{dB})$ & Error $(\mathrm{dB})$ \\
\hline \hline 20 & -26.7 & -25.3 & -1.4 \\
\hline 50 & -19.2 & -17.4 & -1.8 \\
\hline 100 & -13.4 & -11.4 & -2.0 \\
\hline 300 & -4.3 & -2.0 & -2.3 \\
\hline 1000 & 6.1 & 7.3 & -1.2 \\
\hline
\end{tabular}




\subsubsection{Phase Noise Cancellation of 50m SMA Cable System}

The fifth system consisted of $50 \mathrm{~m}$ of RG-58 SMA Cable, a ZRL-1150LN+ amplifier, and an attenuator. The response of the system at various frequencies was measured using a Vector Network Analyzer (VNA) as seen below in Table 32, where $0 \mathrm{kHz}$ offset is $915 \mathrm{MHz}$.

Table 32: System Response Measurements Of 50m SMA Cable

\begin{tabular}{|c|c|c|}
\hline Offset Frequency $(\mathrm{kHz})$ & Amplitude Response $(\mathrm{dB})$ & Phase Response $\left(^{\circ}\right)$ \\
\hline \hline-1000 & -17.604 & 29.126 \\
\hline-300 & -17.667 & -25.615 \\
\hline-100 & -17.692 & -54.125 \\
\hline-50 & -17.701 & -58.75 \\
\hline-20 & -17.71 & -61.537 \\
\hline 0 & -17.712 & -63.268 \\
\hline 20 & -17.719 & -65.225 \\
\hline 50 & -17.727 & -67.991 \\
\hline 100 & -17.709 & -72.6 \\
\hline 300 & -17.735 & -91.059 \\
\hline 1000 & -17.83 & -155.47 \\
\hline
\end{tabular}

Using equation 57, and the system responses measured at each frequency offset, the change in phase noise spectral density relative to the carrier was calculated. The results for which can be seen below in Table 33 . 
Table 33: Phase Noise Spectral Density of 50m system

\begin{tabular}{|c|c|}
\hline Offset Frequency $(\mathrm{kHz})$ & Calculated Change In Phase Noise $(\mathrm{dB})$ \\
\hline \hline 20 & -26.7 \\
\hline 50 & -18.9 \\
\hline 100 & -12.8 \\
\hline 300 & -1.9 \\
\hline 1000 & 6.2 \\
\hline
\end{tabular}

Next the system as implemented with the IQ Mixer, the input power to the RF port of the IQ mixer was measured to be $3.7 \mathrm{dBm}$ as seen below in Figure 77 , in order to ensure it was below the maximum allowable value of $4.2 \mathrm{dBm}$.

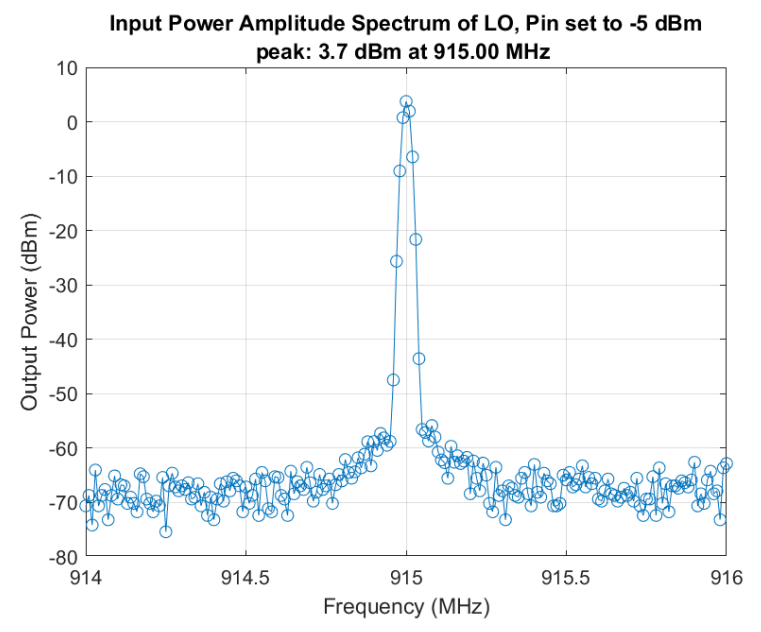

Figure 77: RF Input Power of 50m System

The system was then fully implemented with the IQ mixer, the resulting baseband phase noise can be seen below in Figure 78 and the zoomed in spectrum between $-100 \mathrm{kHz}$ and $100 \mathrm{kHz}$ can be seen in Figure 79 . 


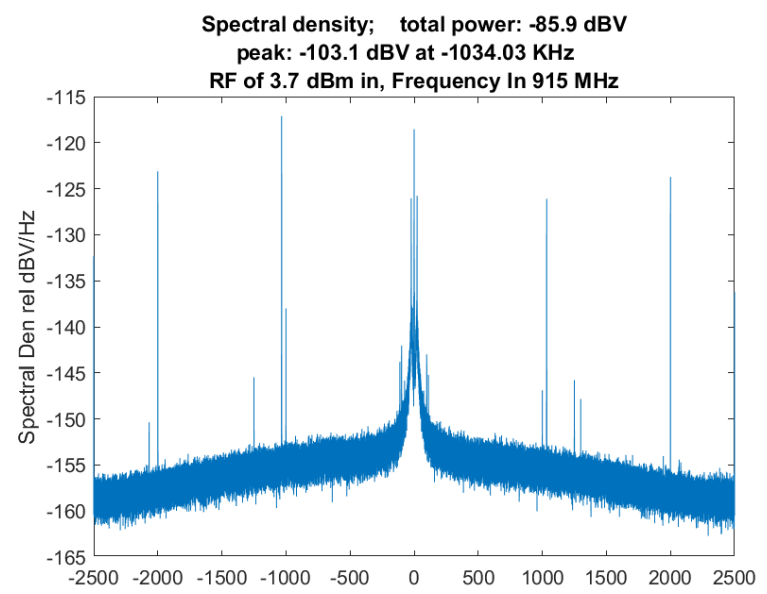

Figure 78: Phase Noise Spectral Density of 50m System

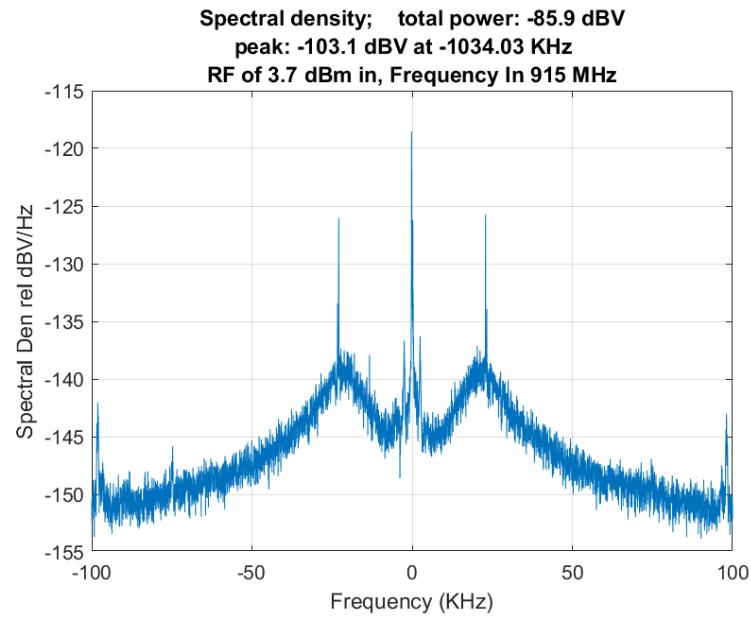

Figure 79: Phase Noise Spectral Density of 50m System From -100 kHz to $100 \mathrm{kHz}$

From the zoomed in spectrum in Figure 79 an unidentified signal can be seen around $95 \mathrm{kHz}$, approximately $8 \mathrm{~dB}$ higher than the noise floor. From this spectrum the new noise floor was measured to be $-158.3 \mathrm{dBV} / \mathrm{Hz}$, compared to the previous $164.4 \mathrm{dBV} / \mathrm{Hz}$. The added noise spectral density was calculated and added to the previously measured values of the noise floor in Table 5, resulting in the new spectral noise density values seen below in 
Table 34 .

Table 34: 50m Cable Noise Floor Spectral Density With RF Input Terminated

\begin{tabular}{|c|c|}
\hline Offset Frequency $(\mathrm{kHz})$ & Calculated Noise Floor $(\mathrm{dBV} / \mathrm{Hz})$ \\
\hline \hline 20 & -155.6 \\
\hline 50 & -157.1 \\
\hline 100 & -157.6 \\
\hline 300 & -158.1 \\
\hline 1000 & -158.3 \\
\hline
\end{tabular}

From this spectrum the spectral density was measured at the same frequencies for which the phase noise was characterized, the resulting values can be seen below in Table 35 . The linear equivalent power of the noise floor values in Table 34 were subtracted from each of the measured values and can also be seen in Table 35 .

Table 35: Phase Noise Of 50m SMA Cable

\begin{tabular}{|c|c|c|}
\hline Offset Frequency $(\mathrm{kHz})$ & Measured PN Spectral Density $(\mathrm{dBV} / \mathrm{Hz})$ & PN Spectral Density Subtracting Noise Floor $(\mathrm{dBV} / \mathrm{Hz})$ \\
\hline \hline 20 & -139.2 & -139.3 \\
\hline 50 & -147.2 & -147.7 \\
\hline 100 & -151.0 & -152.1 \\
\hline 300 & -153.8 & -155.8 \\
\hline 1000 & -155.5 & -158.7 \\
\hline
\end{tabular}

The carrier power of $-20.7 \mathrm{dBV}(3.7 \mathrm{dBV}-24.4 \mathrm{dBV} / \mathrm{dBm})$ was then subtracted from the newly calculated value accounting for the noise floor in order to calculated the phase noise relative to the carrier signal, the resulting values can be seen below in Table 36 . 
Table 36: Phase Noise Spectral Density of 50m system

\begin{tabular}{|c|c|}
\hline Offset Frequency $(\mathrm{kHz})$ & PN Relative to Carrier $(\mathrm{dBC} / \mathrm{Hz})$ \\
\hline \hline 20 & -118.6 \\
\hline 50 & -127.0 \\
\hline 100 & -131.4 \\
\hline 300 & -135.1 \\
\hline 1000 & -138 \\
\hline
\end{tabular}

The phase noise of the TPI synthesizer was then subtracted from these measured phase noises in order to calculate the magnitude of the phase noise cancellation, the result can be seen below in Table 37. The error between the measured and calculated cancellation was calculated and is also shown below in Table 37.

Table 37: Cancellation Measurement for 50m Of Cable

\begin{tabular}{|c|c|c|c|}
\hline Offset Frequency $(\mathrm{kHz})$ & Measured Change In PN $(\mathrm{dB})$ & Calculated Change In PN $(\mathrm{dB})$ & Error $(\mathrm{dB})$ \\
\hline \hline 20 & -28.8 & -26.7 & -2.1 \\
\hline 50 & -20.7 & -18.8 & -1.9 \\
\hline 100 & -15.2 & -12.8 & -2.4 \\
\hline 300 & -7 & -3.4 & -3.6 \\
\hline 1000 & 2.8 & 6.2 & -3.4 \\
\hline
\end{tabular}




\subsubsection{Phase Noise Cancellation of 40m SMA Cable System}

The sixth system consisted of $40 \mathrm{~m}$ of RG-58 SMA Cable, a ZRL-1150LN+ amplifier, and an attenuator. The response of the system at various frequencies was measured using a Vector Network Analyzer (VNA) as seen below in Table 38, where $0 \mathrm{kHz}$ offset is $915 \mathrm{MHz}$.

Table 38: System Response Measurements Of 40m SMA Cable

\begin{tabular}{|c|c|c|}
\hline Offset Frequency $(\mathrm{kHz})$ & Amplitude Response $(\mathrm{dB})$ & Phase Response $\left(^{\circ}\right)$ \\
\hline \hline-1000 & -17.685 & 118.14 \\
\hline-300 & -17.743 & 66.204 \\
\hline-100 & -17.756 & 51.367 \\
\hline-50 & -17.758 & 47.667 \\
\hline-20 & -17.759 & 45.458 \\
\hline 0 & -17.759 & 43.97 \\
\hline 20 & -17.762 & 42.503 \\
\hline 50 & -17.766 & 20.281 \\
\hline 100 & -17.771 & 36.569 \\
\hline 300 & -17.790 & 21.747 \\
\hline 1000 & -17.866 & -30.027 \\
\hline
\end{tabular}

Using equation 57, and the system responses measured at each frequency offset, the change in phase noise spectral density relative to the carrier was calculated. The results for which can be seen below in Table 39 . 
Table 39: Phase Noise Spectral Density of 40m system

\begin{tabular}{|c|c|}
\hline Offset Frequency $(\mathrm{kHz})$ & Calculated Change In Phase Noise $(\mathrm{dB})$ \\
\hline \hline 20 & -28.8 \\
\hline 50 & -20.8 \\
\hline 100 & -14.8 \\
\hline 300 & -5.3 \\
\hline 1000 & 4.6 \\
\hline
\end{tabular}

Next the system as implemented with the IQ Mixer, the input power to the RF port of the IQ mixer was measured to be $3.7 \mathrm{dBm}$ as seen below in Figure 80, in order to ensure it was below the maximum allowable value of $4.2 \mathrm{dBm}$.

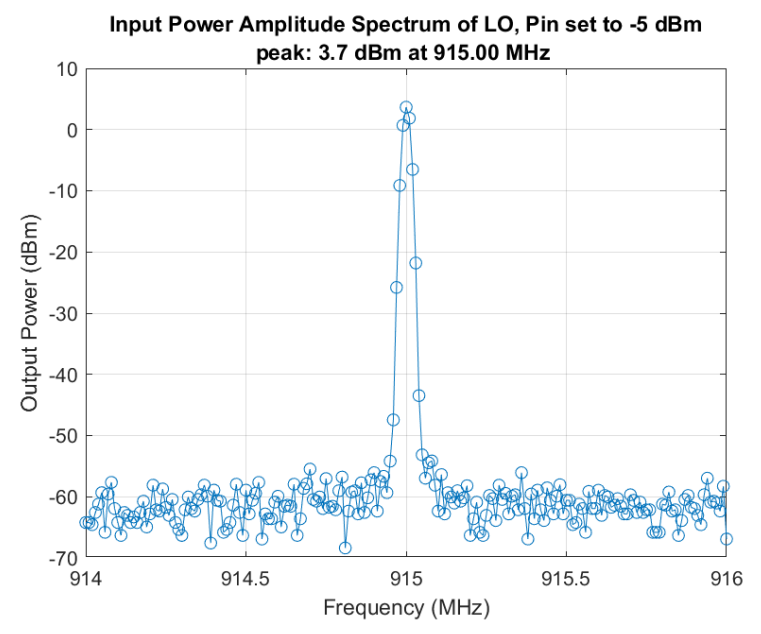

Figure 80: RF Input Power of 40m System

The system was then fully implemented with the IQ mixer, the resulting baseband phase noise can be seen below in Figure 81 and the zoomed in spectrum between $-100 \mathrm{kHz}$ and $100 \mathrm{kHz}$ can be seen in Figure 82 . 


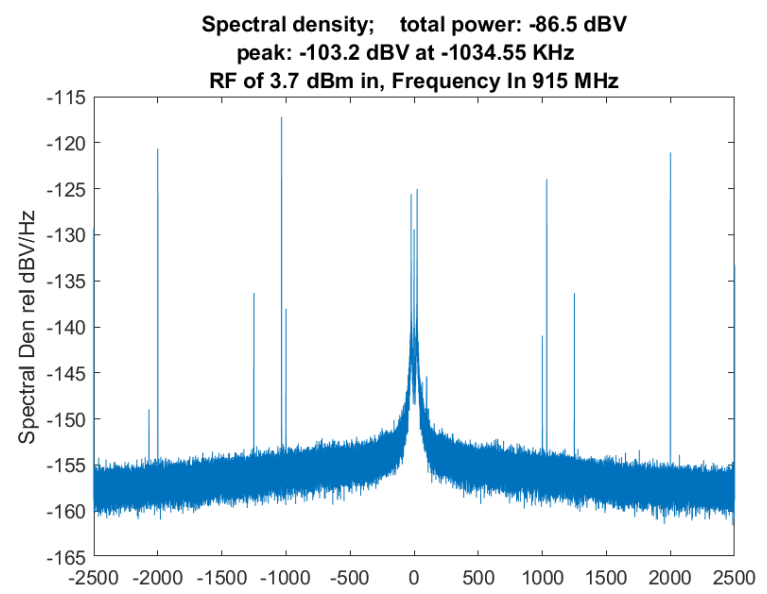

Figure 81: Phase Noise Spectral Density of 40m System

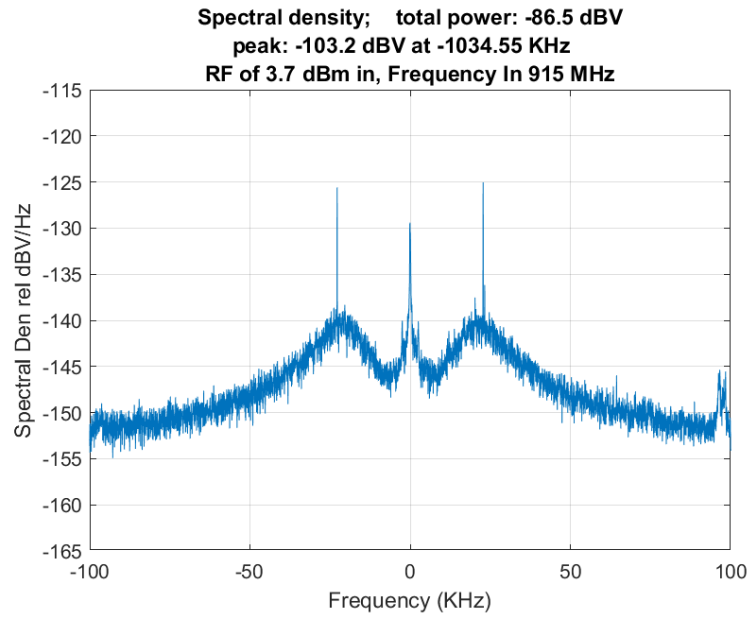

Figure 82: Phase Noise Spectral Density of 40m System From -100 kHz to $100 \mathrm{kHz}$

From the zoomed in spectrum in Figure 79 an unidentified signal can be seen around $95 \mathrm{kHz}$, approximately $5 \mathrm{~dB}$ higher than the noise floor. From this spectrum the new noise floor was measured to be $-157.8 \mathrm{dBV} / \mathrm{Hz}$, compared to the previous $164.4 \mathrm{dBV} / \mathrm{Hz}$. The added noise spectral density was calculated and added to the previously measured values of the noise floor in Table 5, resulting in the new spectral noise density values seen below in 
Table 40.

Table 40: 40m Cable Noise Floor Spectral Density With RF Input Terminated

\begin{tabular}{|c|c|}
\hline Offset Frequency $(\mathrm{kHz})$ & Calculated Noise Floor $(\mathrm{dBV} / \mathrm{Hz})$ \\
\hline \hline 20 & -155.3 \\
\hline 50 & -156.7 \\
\hline 100 & -157.2 \\
\hline 300 & -157.6 \\
\hline 1000 & -157.8 \\
\hline
\end{tabular}

From this spectrum the spectral density was measured at the same frequencies for which the phase noise was characterized, the resulting values can be seen below in Table 41. The linear equivalent power of the noise floor values in Table 40 were subtracted from each of the measured values and can also be seen in Table 41.

Table 41: Phase Noise Of 40m SMA Cable

\begin{tabular}{|c|c|c|}
\hline Offset Frequency $(\mathrm{kHz})$ & Measured PN Spectral Density $(\mathrm{dBV} / \mathrm{Hz})$ & PN Spectral Density Subtracting Noise Floor $(\mathrm{dBV} / \mathrm{Hz})$ \\
\hline \hline 20 & -140.7 & -140.9 \\
\hline 50 & -148.5 & -149.2 \\
\hline 100 & -152.1 & -153.7 \\
\hline 300 & -154.3 & -157.0 \\
\hline 1000 & -156.2 & -161.3 \\
\hline
\end{tabular}

The carrier power of $-20.7 \mathrm{dBV}(4.1 \mathrm{dBV}-24.4 \mathrm{dBV} / \mathrm{dBm})$ was then subtracted from the newly calculated value accounting for the noise floor in order to calculated the phase noise relative to the carrier signal, the resulting values can be seen below in Table 42 . 
Table 42: Phase Noise Spectral Density of 40m system

\begin{tabular}{|c|c|}
\hline Offset Frequency $(\mathrm{kHz})$ & PN Relative to Carrier $(\mathrm{dBC} / \mathrm{Hz})$ \\
\hline \hline 20 & -120.2 \\
\hline 50 & -128.5 \\
\hline 100 & -153.7 \\
\hline 300 & -157.0 \\
\hline 1000 & -161.3 \\
\hline
\end{tabular}

The phase noise of the TPI synthesizer was then subtracted from these measured phase noises in order to calculate the magnitude of the phase noise cancellation, the result can be seen below in Table 43. The error between the measured and calculated cancellation was calculated and is also shown below in Table 43 .

Table 43: Cancellation Measurement for 40m Of Cable

\begin{tabular}{|c|c|c|c|}
\hline Offset Frequency $(\mathrm{kHz})$ & Measured Change In PN $(\mathrm{dB})$ & Calculated Change In PN $(\mathrm{dB})$ & Error $(\mathrm{dB})$ \\
\hline \hline 20 & -30.4 & -28.8 & -1.6 \\
\hline 50 & -22.2 & -20.8 & -1.4 \\
\hline 100 & -16.8 & -14.8 & -2.0 \\
\hline 300 & -8.2 & -5.3 & -2.9 \\
\hline 1000 & 0.2 & 4.6 & -4.4 \\
\hline
\end{tabular}




\subsubsection{Phase Noise Cancellation of 30m SMA Cable System}

The seventh system consisted of $30 \mathrm{~m}$ of RG-58 SMA Cable, a ZRL-1150LN+ amplifier, and an attenuator. The response of the system at various frequencies was measured using a Vector Network Analyzer (VNA) as seen below in Table 44, where $0 \mathrm{kHz}$ offset is $915 \mathrm{MHz}$.

Table 44: System Response Measurements Of 30m SMA Cable

\begin{tabular}{|c|c|c|}
\hline Offset Frequency $(\mathrm{kHz})$ & Amplitude Response $(\mathrm{dB})$ & Phase Response $\left(^{\circ}\right)$ \\
\hline \hline-1000 & 18.233 & -99.805 \\
\hline-300 & -18.270 & -139.19 \\
\hline-100 & -18.276 & -150.4 \\
\hline-50 & -18.277 & -153.17 \\
\hline-20 & -18.275 & -154.75 \\
\hline 0 & -18.274 & -155.92 \\
\hline 20 & -18.274 & -156.81 \\
\hline 50 & -18.275 & -158.87 \\
\hline 100 & -18.275 & -161.41 \\
\hline 300 & 18.300 & -172.56 \\
\hline 1000 & -18.357 & 148.9 \\
\hline
\end{tabular}

Using equation 57, and the system responses measured at each frequency offset, the change in phase noise spectral density relative to the carrier was calculated. The results for which can be seen below in Table 45 . 
Table 45: Phase Noise Spectral Density of 30m system

\begin{tabular}{|c|c|}
\hline Offset Frequency $(\mathrm{kHz})$ & Calculated Change In Phase Noise $(\mathrm{dB})$ \\
\hline \hline 20 & -31.8 \\
\hline 50 & -23.1 \\
\hline 100 & -17.3 \\
\hline 300 & -7.7 \\
\hline 1000 & 2.4 \\
\hline
\end{tabular}

Next the system as implemented with the IQ Mixer, the input power to the RF port of the IQ mixer was measured to be $3.3 \mathrm{dBm}$ as seen below in Figure 83, in order to ensure it was below the maximum allowable value of $4.2 \mathrm{dBm}$.

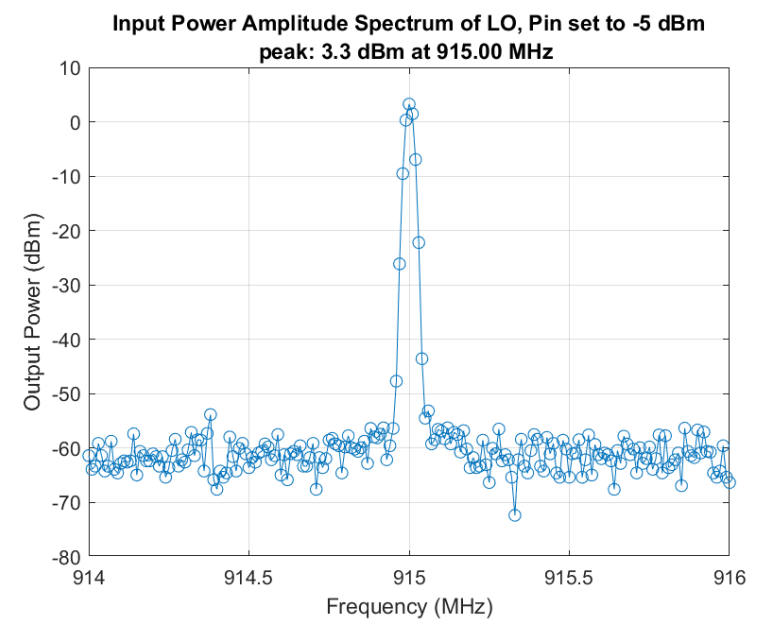

Figure 83: RF Input Power of 30m System

The system was then fully implemented with the IQ mixer, the resulting baseband phase noise can be seen below in Figure 84 and the zoomed in spectrum between $-100 \mathrm{kHz}$ and $100 \mathrm{kHz}$ can be seen in Figure 85 . 


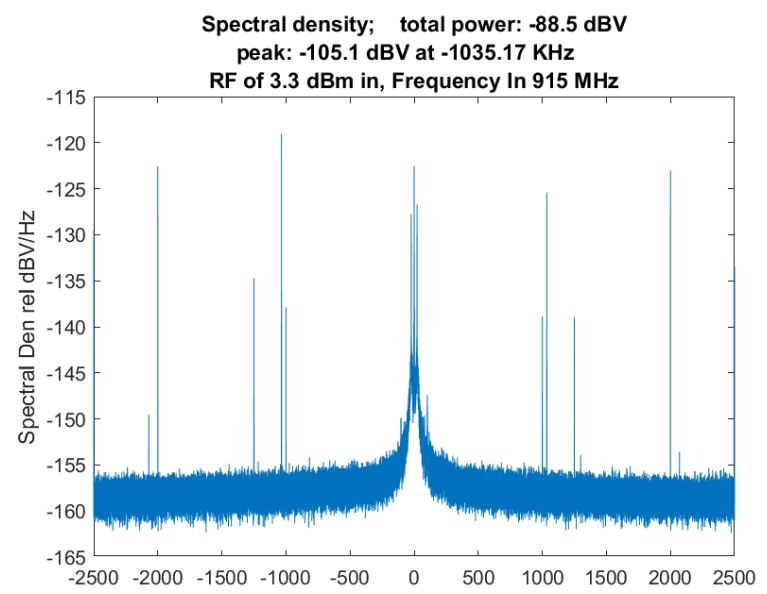

Figure 84: Phase Noise Spectral Density of 30m System

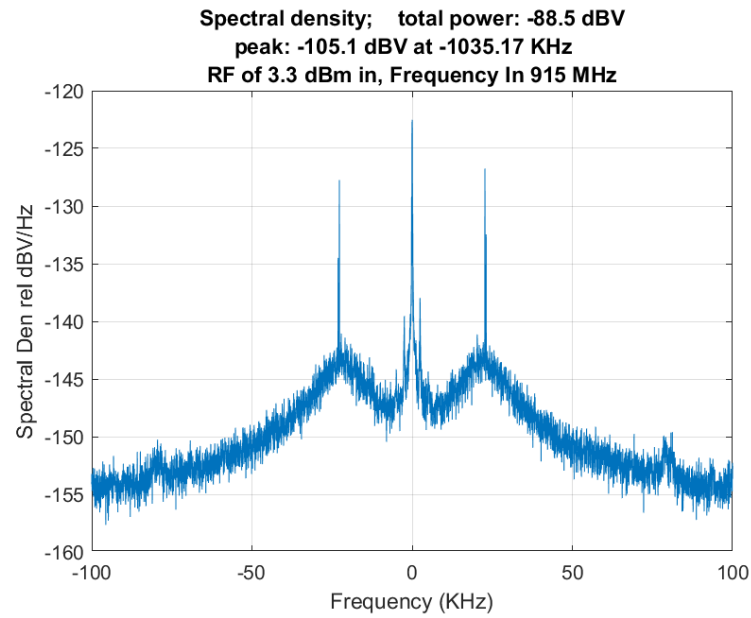

Figure 85: Phase Noise Spectral Density of 30m System From -100 kHz to $100 \mathrm{kHz}$

From this spectrum the new noise floor was measured to be $-158.8 \mathrm{dBV} / \mathrm{Hz}$, compared to the previous $164.4 \mathrm{dBV} / \mathrm{Hz}$. The added noise spectral density was calculated and added to the previously measured values of the noise floor in Table 5 , resulting in the new spectral noise density values seen below in Table 46 . 
Table 46: 30m Cable Noise Floor Spectral Density With RF Input Terminated

\begin{tabular}{|c|c|}
\hline Offset Frequency $(\mathrm{kHz})$ & Calculated Noise Floor $(\mathrm{dBV} / \mathrm{Hz})$ \\
\hline \hline 20 & -155.9 \\
\hline 50 & -157.4 \\
\hline 100 & -158.0 \\
\hline 300 & -158.6 \\
\hline 1000 & -158.8 \\
\hline
\end{tabular}

From the zoomed in spectrum in Figure 79 an unidentified signal can be seen around $80 \mathrm{kHz}$, approximately $3 \mathrm{~dB}$ higher than the noise floor. From this spectrum the spectral density was measured at the same frequencies for which the phase noise was characterized, the resulting values can be seen below in Table 47. The linear equivalent power of the noise floor values in Table 46 were subtracted from each of the measured values and can also be seen in Table 47.

Table 47: Phase Noise Of 30m SMA Cable

\begin{tabular}{|c|c|c|}
\hline Offset Frequency $(\mathrm{kHz})$ & Measured PN Spectral Density $(\mathrm{dBV} / \mathrm{Hz})$ & PN Spectral Density Subtracting Noise Floor $(\mathrm{dBV} / \mathrm{Hz})$ \\
\hline \hline 20 & -143.3 & -143.5 \\
\hline 50 & -151.0 & -152.1 \\
\hline 100 & -154.3 & -156.7 \\
\hline 300 & -156.8 & -161.5 \\
\hline 1000 & -158.0 & -165.7 \\
\hline
\end{tabular}

The carrier power of $-21.1 \mathrm{dBV}(3.3 \mathrm{dBV}-24.4 \mathrm{dBV} / \mathrm{dBm})$ was then subtracted from the newly calculated value accounting for the noise floor in order to calculated the phase noise relative to the carrier signal, the resulting values can be seen below in Table 48 . 
Table 48: Phase Noise Spectral Density of 30m system

\begin{tabular}{|c|c|}
\hline Offset Frequency $(\mathrm{kHz})$ & PN Relative to Carrier $(\mathrm{dBC} / \mathrm{Hz})$ \\
\hline \hline 20 & -122.4 \\
\hline 50 & -131.0 \\
\hline 100 & -135.6 \\
\hline 300 & -140.4 \\
\hline 1000 & -144.6 \\
\hline
\end{tabular}

The phase noise of the TPI synthesizer was then subtracted from these measured phase noises in order to calculate the magnitude of the phase noise cancellation, the result can be seen below in Table 49. The error between the measured and calculated cancellation was calculated and is also shown below in Table 49 .

Table 49: Cancellation Measurement for 30m Of Cable

\begin{tabular}{|c|c|c|c|}
\hline Offset Frequency $(\mathrm{kHz})$ & Measured Change In PN $(\mathrm{dB})$ & Calculated Change In PN $(\mathrm{dB})$ & Error $(\mathrm{dB})$ \\
\hline \hline 20 & -32.6 & -31.8 & -0.8 \\
\hline 50 & -24.7 & -23.1 & -1.6 \\
\hline 100 & -19.4 & -17.3 & -2.1 \\
\hline 300 & -12.3 & -7.7 & -4.6 \\
\hline 1000 & -3.8 & 2.4 & -6.2 \\
\hline
\end{tabular}




\subsubsection{Cancellation Overview}

This subsection will provide an overview of the measurements at each frequency offset versus cable length as well as a system wide discussion. It will also discuss the differences between the measured and expected values, and will cover possible sources of error which may contribute to these differences.

The measured and calculated change in phase noise for a $20 \mathrm{kHz}$ offset from the carrier can be seen below in Table 50 as well as the error between the two.

Table 50: Calculated and Measured Change in Phase Noise at $20 \mathrm{KHz}$ Offset

\begin{tabular}{|c|c|c|c|}
\hline Cable $(\mathrm{m})$ & Calculated Cancellation $(\mathrm{dB})$ & Measured Cancellation $(\mathrm{dB})$ & Error $(\mathrm{dB})$ \\
\hline \hline 90 & -22.2 & -24.1 & -1.9 \\
\hline 80 & -23.1 & -24.8 & -1.7 \\
\hline 70 & -23.7 & -25.8 & -2.1 \\
\hline 60 & -25.3 & -26.7 & -1.4 \\
\hline 50 & -26.7 & -28.8 & -2.1 \\
\hline 40 & -28.8 & -30.4 & -1.6 \\
\hline 30 & -31.8 & -32.6 & -0.8 \\
\hline
\end{tabular}

The measured and calculated change in phase noise spectral density can also be seen plotted below in Figure 86 . 


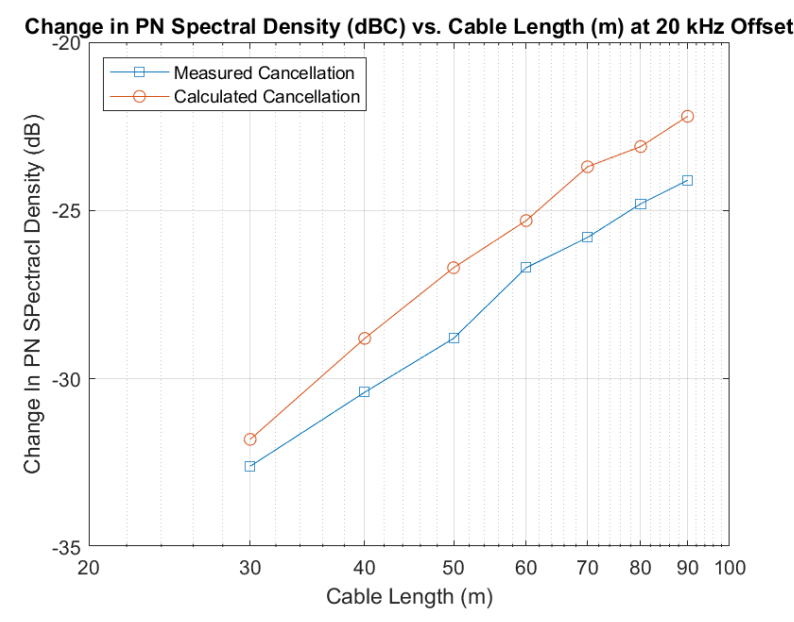

Figure 86: Change In Phase Noise Spectral Density Relative to Carrier at $20 \mathrm{kHz}$ Offset

The measured change in phase noise is consistently below the calculated value by an average of approximately $1.9 \mathrm{~dB}$. While the magnitude of the change is offset the rate of change in phase noise can be seen approximately the same between the measured and calculated values. The error between the measured and calculated value seems to lower with the $30 \mathrm{~m}$ cable, however this is likely due to the fact that the phase difference between the carrier and offset is very small at approximately $1^{\circ}$. A small error in the measured phase response of 0.2 degrees could change the estimated cancellation significantly. For example if the phase at $-20 \mathrm{kHz}$ offset was measured to be $-154.65^{\circ}$ instead of the measured $-154.75^{\circ}$ and the phase at $+20 \mathrm{kHz}$ offset was measured to be $-156.91^{\circ}$ instead of the measured $-156.81^{\circ}$ the calculated cancellation would be $-31.0 \mathrm{~dB}$, resulting in an error or $-1.6 \mathrm{~dB}$ instead of the current $-0.8 \mathrm{~dB}$.

The measured and calculated change in phase noise for a $50 \mathrm{kHz}$ offset and error between the two can be seen below in Table 51 and plotted in Figure 87. 
Table 51: Calculated and Measured Change in Phase Noise at $50 \mathrm{KHz}$ Offset

\begin{tabular}{|c|c|c|c|}
\hline Cable $(\mathrm{m})$ & Calculated Cancellation $(\mathrm{dB})$ & Measured Cancellation $(\mathrm{dB})$ & Error $(\mathrm{dB})$ \\
\hline \hline 90 & -14.3 & -15.4 & -1.1 \\
\hline 80 & -15.2 & -16.9 & -1.7 \\
\hline 70 & -16.1 & -18.1 & -2.0 \\
\hline 60 & -17.4 & -19.2 & -1.8 \\
\hline 50 & -18.8 & -20.7 & -1.9 \\
\hline 40 & -20.8 & -22.2 & -1.4 \\
\hline 30 & -23.1 & -24.7 & -1.6 \\
\hline
\end{tabular}

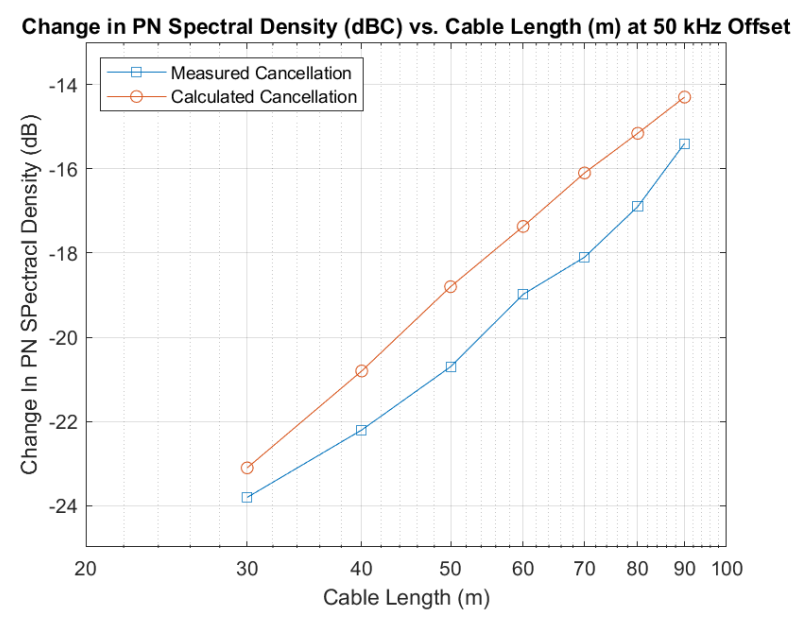

Figure 87: Change In Phase Noise Spectral Density Relative to Carrier at $50 \mathrm{kHz}$ Offset

Once again the rate of change between the calculated and measured values is approximately the same, however the average offset is slightly different at approximately $1.7 \mathrm{~dB}$.

The measured and calculated change in phase noise for a $100 \mathrm{kHz}$ offset and error between the two can be seen below in Table 52 and plotted in Figure 88. 
Table 52: Calculated and Measured Change in Phase Noise at $100 \mathrm{KHz}$ Offset

\begin{tabular}{|c|c|c|c|}
\hline Cable $(\mathrm{m})$ & Calculated Cancellation $(\mathrm{dB})$ & Measured Cancellation $(\mathrm{dB})$ & Error $(\mathrm{dB})$ \\
\hline \hline 90 & -8.3 & -9.7 & -1.4 \\
\hline 80 & -9.1 & -11.2 & -2.1 \\
\hline 70 & -10.1 & -12.1 & -2.0 \\
\hline 60 & -11.4 & -13.4 & -2.0 \\
\hline 50 & -12.8 & -15.2 & -2.4 \\
\hline 40 & -14.8 & -16.8 & -2.0 \\
\hline 30 & -17.3 & -19.4 & -2.1 \\
\hline
\end{tabular}

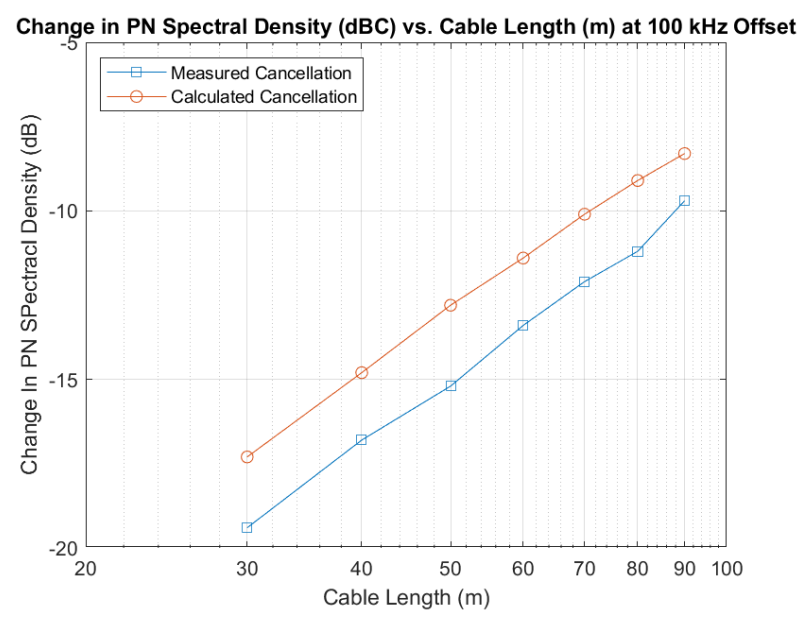

Figure 88: Change In Phase Noise Spectral Density Relative to Carrier at $100 \mathrm{kHz}$ Offset

The rate of change between the calculated and measured values is also approximately the same, however the average offset is slightly different at approximately $2.0 \mathrm{~dB}$.

The measured and calculated change in phase noise for a $300 \mathrm{kHz}$ offset and error between the two can be seen below in Table 53 and plotted in Figure 89. 
Table 53: Calculated and Measured Change in Phase Noise at $300 \mathrm{KHz}$ Offset

\begin{tabular}{|c|c|c|c|}
\hline Cable $(\mathrm{m})$ & Calculated Cancellation $(\mathrm{dB})$ & Measured Cancellation $(\mathrm{dB})$ & Error $(\mathrm{dB})$ \\
\hline \hline 90 & 1.0 & -0.4 & -1.4 \\
\hline 80 & 0.2 & -1.8 & -2.0 \\
\hline 70 & -0.7 & -2.8 & -2.1 \\
\hline 60 & -2.0 & -4.3 & -2.3 \\
\hline 50 & -3.4 & -7.0 & -3.6 \\
\hline 40 & -5.3 & -8.2 & -2.9 \\
\hline 30 & -7.7 & -12.3 & -4.6 \\
\hline
\end{tabular}

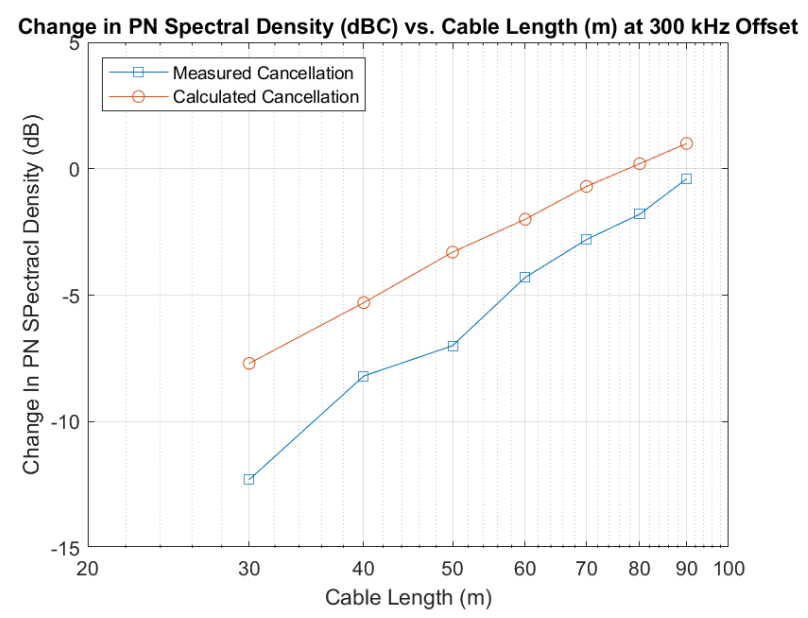

Figure 89: Change In Phase Noise Spectral Density Relative to Carrier at $300 \mathrm{kHz}$ Offset

The rate of change between the calculated and measured values is also nearly the same for cables approximately $60 \mathrm{~m}$ and longer at an error of approximately $2.0 \mathrm{~dB}$. At cables lengths of $50 \mathrm{~m}$ or shorter the calculated values remain at the same slope, however the measured values begin to lower at a much faster rate. This is likely due to the fact the signal is very close to the noise floor. Therefore a small error in the initial measurement can cause a significant error once the noise floor has been subtracted. For example if the 
measured value for the $50 \mathrm{~m}$ cable was $-153.6 \mathrm{dBV} / \mathrm{Hz}$ instead of $-153.8 \mathrm{dBV} / \mathrm{Hz}$ the resulting spectral density without the noise floor would be $-153.5 \mathrm{dBV} / \mathrm{Hz}$, or a total change of -3.4 $\mathrm{dBV}$. Additionally the noise floor for this measurement was the highest measured, if it was instead measured at $-158.8 \mathrm{dBV} / \mathrm{Hz}$, the next highest noise floor measured, this would result in a measurement of $-155.2 \mathrm{dBV} / \mathrm{Hz}$ or a $-3.1 \mathrm{~dB}$ change in the phase noise, closer to the expected range.

The measured and calculated change in phase noise for a $1000 \mathrm{kHz}$ offset and error between the two can be seen below in Table 54 and plotted in Figure 90.

Table 54: Calculated and Measured Change in Phase Noise at $1000 \mathrm{KHz}$ Offset

\begin{tabular}{|c|c|c|c|}
\hline Cable $(\mathrm{m})$ & Calculated Cancellation $(\mathrm{dB})$ & Measured Cancellation $(\mathrm{dB})$ & Error $(\mathrm{dB})$ \\
\hline \hline 90 & 8.8 & 8.6 & -0.2 \\
\hline 80 & 8.5 & 8.3 & -0.2 \\
\hline 70 & 8.0 & 7.6 & -0.4 \\
\hline 60 & 7.3 & 6.1 & -1.2 \\
\hline 50 & 6.2 & 2.8 & -3.4 \\
\hline 40 & 4.6 & 0.2 & -4.4 \\
\hline 30 & 2.4 & -3.8 & -6.2 \\
\hline
\end{tabular}




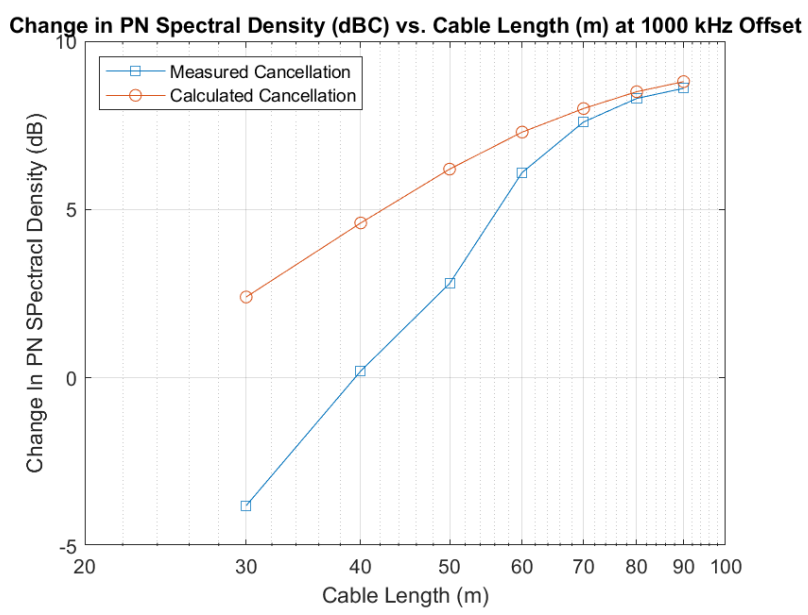

Figure 90: Change In Phase Noise Spectral Density Relative to Carrier at $1000 \mathrm{kHz}$ Offset

Similar to the measurements of the $300 \mathrm{kHz}$ offset at short cable lengths the measured value begins to rapidly deviate from the expected value, this may be due to the fact the measured values are also approaching the noise floor.

In addition two system wide sources of error were noted, the first of which was the method used for measuring the spectral density of the phase noise within the spectrum. Due to the phase noise being relatively close to the thermal noise floor there was significant fluctuation in spectral density during measurement. When taking the measurement at a frequency the approximate average of this fluctuation was taken in $\mathrm{dB}$, not in the linear equivalent. From the various measurements of the cancelled phase noise an approximate range of $\pm 2.5 \mathrm{~dB}$ can be taken. If the values selected were $0 \mathrm{~dB}(1 \mathrm{~W})$ and $5 \mathrm{~dB}(3.1623 \mathrm{~W})$ the $\mathrm{dB}$ average would be $2.5 \mathrm{~dB}$, however the linear average would be $2.0811 \mathrm{~W}$, or 3.1830 $\mathrm{dB}$ - a difference of $0.68 \mathrm{~dB}$. This same issue would take place in the original measurements of the phase noise, however due to the higher power of the phase noise itself the fluctuation would be less on a dB scale. Using Figure 59 an approximate fluctuation of $\pm 1.5 \mathrm{~dB}$ can be seen. If the values selected were $0 \mathrm{~dB}(1 \mathrm{~W})$ and $3 \mathrm{~dB}(2 \mathrm{~W})$ the $\mathrm{dB}$ average would be $1.5 \mathrm{~dB}$, however the linear average would be $1.5 \mathrm{~W}$, or $1.7609 \mathrm{~dB}$ - a difference of 0.26 
$\mathrm{dB}$. Therefore this could increase the measured values by approximately $0.4 \mathrm{~dB}$. This value would change depending on which cable and frequency offset was being measured, in order to reduce this error the number of averaged captures could be increased, this would reduce the overall fluctuation of the noise.

A second source of error noted was the measured input and output power of the system under test when compared to the expected output power using the input power and measured system amplitude response. While the VNA was calibrated prior to measurement it was noted that the $\mathrm{CMU}$ was not recently calibrated. Table 55 below shows the measured output power of the system and the expected output power of the system, calculated using the the measured input power of $20.0 \mathrm{dBm}$ and the measured amplitude response of the system at the carrier frequency, as well as the error between the two.

Table 55: Expected and Measured Output Power of Systems Under Test

\begin{tabular}{|c|c|c|c|}
\hline Cable Length $(\mathrm{m})$ & Expected Output Power $(\mathrm{dBm})$ & Measured Output Power $(\mathrm{dBm})$ & Error $(\mathrm{dB})$ \\
\hline \hline 30 & 1.7 & 3.3 & 1.6 \\
\hline 40 & 2.2 & 3.7 & 1.5 \\
\hline 50 & 2.3 & 3.7 & 1.4 \\
\hline 60 & 3.7 & 4.1 & 0.4 \\
\hline 70 & 3.2 & 3.5 & 0.3 \\
\hline 80 & 3.2 & 3.5 & 0.3 \\
\hline 90 & 2.9 & 3.4 & 0.5 \\
\hline
\end{tabular}

If the expected output power value is used to calculated the measured cancellation tables 56 through 60 show the newly calculated values and errors, while figures 91 through 95 show the new plots. 
Table 56: Calculated and Modified Measured Change in Phase Noise at $20 \mathrm{KHz}$ Offset

\begin{tabular}{|c|c|c|c|}
\hline Cable $(\mathrm{m})$ & Calculated Cancellation $(\mathrm{dB})$ & Measured Cancellation $(\mathrm{dB})$ & Error $(\mathrm{dB})$ \\
\hline \hline 90 & -22.2 & -23.6 & -1.4 \\
\hline 80 & -23.1 & -24.5 & -1.4 \\
\hline 70 & -23.7 & -25.5 & -1.8 \\
\hline 60 & -25.3 & -26.3 & -1.0 \\
\hline 50 & -26.7 & -27.4 & -0.7 \\
\hline 40 & -28.8 & -28.9 & -0.1 \\
\hline 30 & -31.8 & -31.0 & 0.8 \\
\hline
\end{tabular}

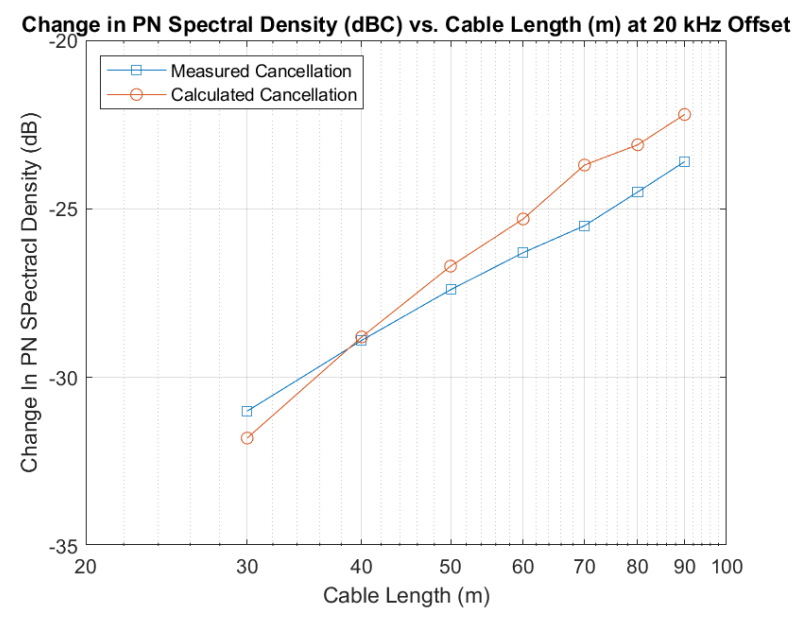

Figure 91: Change In Phase Noise Spectral Density Relative to Carrier at $20 \mathrm{kHz}$ Offset 
Table 57: Calculated and Modified Measured Change in Phase Noise at $50 \mathrm{KHz}$ Offset

\begin{tabular}{|c|c|c|c|}
\hline Cable $(\mathrm{m})$ & Calculated Cancellation $(\mathrm{dB})$ & Measured Cancellation $(\mathrm{dB})$ & Error $(\mathrm{dB})$ \\
\hline \hline 90 & -14.3 & -14.9 & -0.6 \\
\hline 80 & -15.2 & -16.6 & -1.4 \\
\hline 70 & -16.1 & -17.8 & -1.7 \\
\hline 60 & -17.4 & -18.6 & -1.2 \\
\hline 50 & -18.8 & -19.3 & -0.5 \\
\hline 40 & -20.8 & -20.7 & 0.1 \\
\hline 30 & -23.1 & -23.1 & 0.0 \\
\hline
\end{tabular}

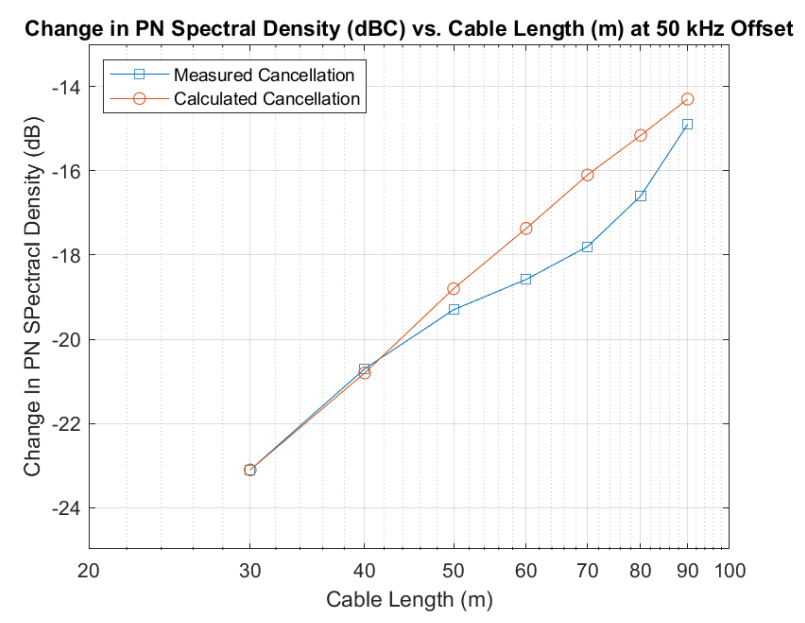

Figure 92: Change In Phase Noise Spectral Density Relative to Carrier at $50 \mathrm{kHz}$ Offset 
Table 58: Calculated and Modified Measured Change in Phase Noise at $100 \mathrm{KHz}$ Offset

\begin{tabular}{|c|c|c|c|}
\hline Cable $(\mathrm{m})$ & Calculated Cancellation $(\mathrm{dB})$ & Measured Cancellation $(\mathrm{dB})$ & Error $(\mathrm{dB})$ \\
\hline \hline 90 & -8.3 & -9.2 & -0.9 \\
\hline 80 & -9.1 & -10.9 & -1.8 \\
\hline 70 & -10.1 & -11.8 & -1.7 \\
\hline 60 & -11.4 & -13.0 & -1.6 \\
\hline 50 & -12.8 & -13.8 & -1.0 \\
\hline 40 & -14.8 & -15.3 & -0.5 \\
\hline 30 & -17.3 & -17.8 & -0.5 \\
\hline
\end{tabular}

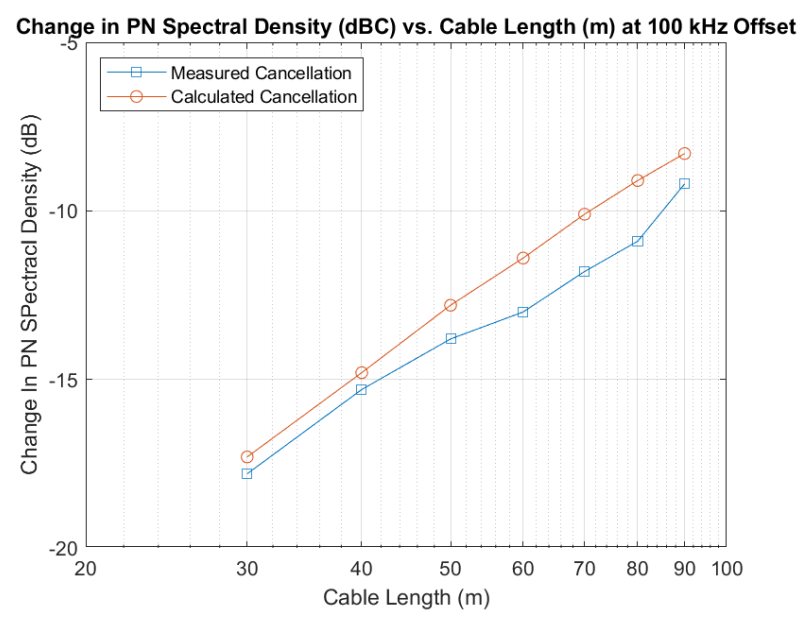

Figure 93: Change In Phase Noise Spectral Density Relative to Carrier at $100 \mathrm{kHz}$ Offset 
Table 59: Calculated and Modified Measured Change in Phase Noise at $300 \mathrm{KHz}$ Offset

\begin{tabular}{|c|c|c|c|}
\hline Cable $(\mathrm{m})$ & Calculated Cancellation $(\mathrm{dB})$ & Measured Cancellation $(\mathrm{dB})$ & Error $(\mathrm{dB})$ \\
\hline \hline 90 & 1.0 & 0.1 & -0.9 \\
\hline 80 & 0.2 & -1.5 & -1.7 \\
\hline 70 & -0.7 & -2.5 & -1.8 \\
\hline 60 & -2.0 & -3.9 & -1.9 \\
\hline 50 & -3.3 & -5.6 & -2.3 \\
\hline 40 & -5.3 & -6.7 & -1.4 \\
\hline 30 & -7.7 & -10.7 & -3.0 \\
\hline
\end{tabular}

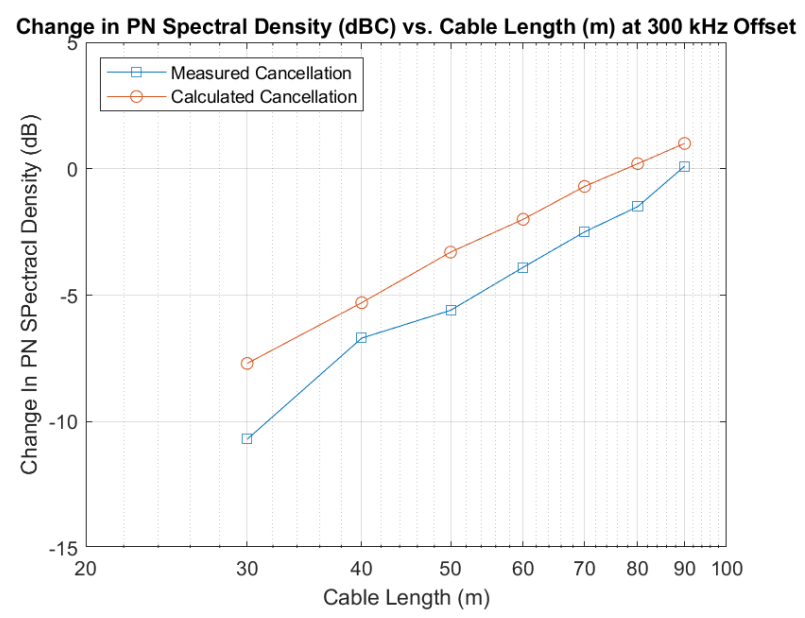

Figure 94: Change In Phase Noise Spectral Density Relative to Carrier at $300 \mathrm{kHz}$ Offset 
Table 60: Calculated and Modified Measured Change in Phase Noise at $1000 \mathrm{KHz}$ Offset

\begin{tabular}{|c|c|c|c|}
\hline Cable $(\mathrm{m})$ & Calculated Cancellation $(\mathrm{dB})$ & Measured Cancellation $(\mathrm{dB})$ & Error $(\mathrm{dB})$ \\
\hline \hline 90 & 8.8 & 9.1 & 0.3 \\
\hline 80 & 8.5 & 8.6 & 0.1 \\
\hline 70 & 8.0 & 7.9 & -0.1 \\
\hline 60 & 7.3 & 6.5 & -0.8 \\
\hline 50 & 6.2 & 4.2 & -2.0 \\
\hline 40 & 4.6 & 1.7 & -2.9 \\
\hline 30 & 2.4 & -2.2 & -4.6 \\
\hline
\end{tabular}

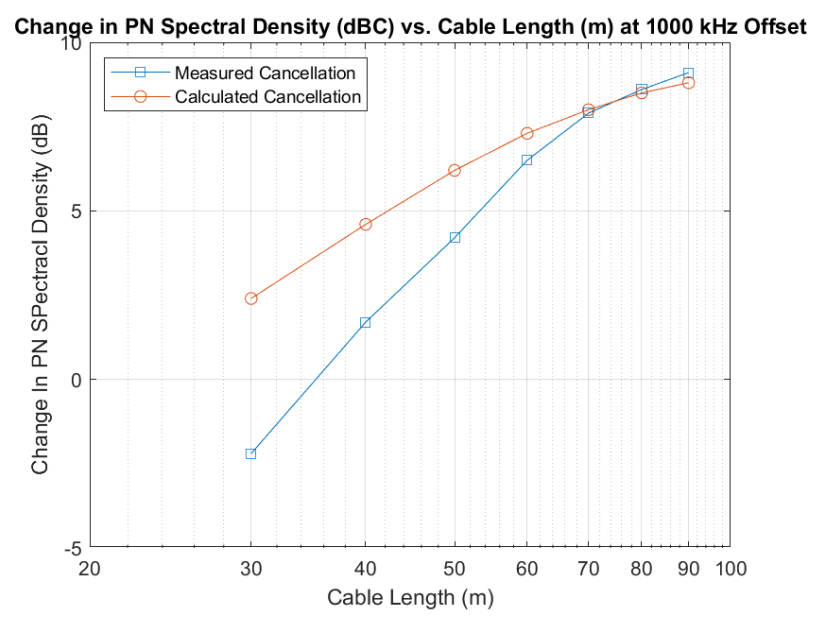

Figure 95: Change In Phase Noise Spectral Density Relative to Carrier at $1000 \mathrm{kHz}$ Offset

This adjustment does significantly decrease the average error between the expected and measured values, however it is also possible the measured input power is incorrect and should be higher, this would cause the error to increase. Therefore it cannot be definitively said that this correction is accurate but is a significant source of error.

In addition to these possible sources of error even though the cables are insulated, given the lengths of cable being used and the low powers being measured, it is possible other 
wireless signals occurring around $915 \mathrm{MHz}$ could have been interfering with measurement. Two unexplained tones were observed at $95 \mathrm{kHz}$ offset and one at $80 \mathrm{kHz}$ offset in the $50 \mathrm{~m}$, $40 \mathrm{~m}$, and $30 \mathrm{~m}$ systems respectively at baseband. While it is possible these were previously unobserved low power spurs, the frequency offset changing may mean it is coming from an outside source. 


\section{Conclusion and Future Work}

\subsection{Contributions}

The analysis and results from this thesis show that this mixer system can reduce the power level of a leaked Local Oscillator phase noise signal in a full duplex transceiver system. This can increase the overall signal-to-noise ratio and subsequently increases maximum sensitivity of the receiver when looking at modulated data surrounding the carrier signal. The system utilizes a simplistic topology providing cost and volume advantages to more complicated phase noise cancellation techniques. The proposed system could also be implemented using purely passive components if power consumption of the transceiver was a major concern. Lastly this method of phase noise cancellation was implemented by Teslonix Inc., using a proprietary tuning method to maximize the magnitude of cancellation.

\subsection{Conclusion}

The ability of the proposed system architecture to cancel phase noise of a leaked Local Oscillator signal into the receiver of a Full-Duplex Transceiver was demonstrated. The predicted and measured values were typically within $\pm 2.5 \mathrm{~dB}$ of each other, with some outliers measured near the noise floor being off up to $6.2 \mathrm{~dB}$. Current solutions to self-interference typically require additional components to utilize their described cancellation method, however the proposed system could use no additional components for minimal cancellation. For example if an integrated circuit were being designed with the system response in mind the transceiver itself could cause cancellation without any additional components. However if maximum cancellation is desired a circuit response tuner would be used in order to align the response of the system at the carrier frequency as well as the offset frequencies of interest. If further analog cancellation is still required the described system could also be used in conjunction with other methods of self-interference cancellation provided they do not inter- 
fere with the assumptions made during analysis: the system under test is linear and time invariant.

\subsection{Future Work}

While the cancellation of the system was shown to be approximately equal to the predicted values using equation 1 additional work should focus on demonstrating the functionality of the system using more complex systems, and improving the accuracy of the measurements in order to reduce the error of measurements. There are a number of improvements that could be made to the system in order to accomplish this. Replacing the mixers could significantly improve the ability to measure the cancellation, using active mixers with a better noise figure or less conversion loss would improve the SNR of the phase noise to the noise floor. Additionally if the mixers are replaced such that they allow higher RF input the measured signal at the output could be increased further away from the noise floor. Another major limiting component is the synthesizers, if they were replaced with synthesizers with higher phase noise, a lower noise floor, or a combination of both, a greater magnitude of cancellation could be measured. Lastly if the implemented components such as power splitters, filters, and baseband amplifiers were replaced with lower noise figure components this would also lower the output noise floor of the system, allowing for a greater measurable phase noise cancellation. As discussed previously if a larger number of captures were averaged the variation of signal power due to noise would decrease, therefore increasing measurement accuracy. 


\section{References}

[1] J. J. Podesta, "Phase noise cancellation in a mixer circuit: Analysis using a random phase function." Army Armament Research Development And Engineering Center, Tech. Rep., 1996.

[2] R. G. Qiu, "Rfid-enabled automation in support of factory integration," Robotics and Computer-Integrated Manufacturing, vol. 23, no. 6, pp. 677-683, 2007.

[3] F. Gandino, B. Montrucchio, M. Rebaudengo, and E. R. Sanchez, "On improving automation by integrating rfid in the traceability management of the agri-food sector," IEEE Transactions on Industrial Electronics, vol. 56, no. 7, pp. 2357-2365, 2009.

[4] D. M. Dobkin, "The rf in rfid passive uhf in practice," United States of America, Newness, 2008.

[5] N. Roberts, "Phase noise and jitter - a primer for digital designers," Electronic Engineering Times, 2003.

[6] W. Kester, "Converting oscillator phase noise to time jitter," Tutorial MT-008, Analog Devices, p. 2009, 2009.

[7] J. W. Rogers and C. Plett, Radio frequency integrated circuit design. Artech House, 2010.

[8] A. M. Niknejad, "Lecture 24: Oscillator phase noise," Lecture Notes, 2005.

[9] J. W. Rogers, C. Plett, and I. Marsland, Radio frequency system architecture and design. Artech House, 2013.

[10] D. Lesson, "A simple model of feedback oscillator noise spectrum," proc. IEEE, vol. 54, no. 2, pp. 329-330, 1966.

[11] M. Haartman and M. Östling, Low-frequency noise in advanced MOS devices. Springer Science \& Business Media, 2007. 
[12] K. K. Hung, P. K. Ko, C. Hu, and Y. C. Cheng, "A unified model for the flicker noise in metal-oxide-semiconductor field-effect transistors," IEEE Transactions on Electron Devices, vol. 37, no. 3, pp. 654-665, 1990.

[13] R. F. Voss, "Linearity of $1 \mathrm{f}$ noise mechanisms," Physical Review Letters, vol. 40, no. 14, p. $913,1978$.

[14] D. V. Perepelitsa, "Johnson noise and shot noise," Dept. of Physics, MIT, 2006.

[15] S. Engelberg, Random signals and noise: a mathematical introduction. CRC Press, 2006.

[16] Keysight Technologies. Phase Noise Measurement Solutions Selection Guide. [Online]. Available: https://literature.cdn.keysight.com/litweb/pdf/5990-5729EN.pdf

[17] D. Owen, "Good practice guide to phase noise measurement," National Physical Laboratory, Middlesex, UK, Tech. Note, vol. 68, 2004.

[18] J. G. Proakis, Digital signal processing: principles algorithms and applications. Pearson Education India, 2001.

[19] National Instruments. Understanding FFTs and Windowing. [Online]. Available: http://download.ni.com/evaluation/pxi/Understanding FFTs and Windowing.pdf

[20] R. Poisel, Target acquisition in communication electronic warfare systems. Artech House, 2003.

[21] J. O. Smith III, Spectral audio signal processing. W3K publishing, 2011.

[22] R. G. Lyons, Understanding digital signal processing, 3/E. Pearson Education India, 2004 .

[23] L. Tan and J. Jiang, Digital signal processing: fundamentals and applications. Academic Press, 2018.

[24] B. Nath, F. Reynolds, and R. Want, "Rfid technology and applications," IEEE Pervasive Computing, vol. 5, no. 1, pp. 22-24, 2006. 
[25] F. Xiong, Digital modulation techniques. Artech house, inc., 2000.

[26] A. Boaventura, J. Santos, A. Oliveira, and N. B. Carvalho, "Perfect isolation: Dealing with self-jamming in passive rfid systems," IEEE Microwave Magazine, vol. 17, no. 11, pp. 20-39, 2016.

[27] G. S. Sahota and C. Komninakis, "Baseband transmitter self-jamming and intermodulation cancellation device," U.S. Patent 8170 487B2, 2006.

[28] B. Rothenberg, "Full duplex transceiver having a method for immunizing itself against self-jamming," U.S. Patent 7155 179B1, 2001.

[29] Z. Chen, D. Liao, and F. F. Dai, "A full-duplex transceiver front-end rfic with codedomain spread spectrum modulation for tx self-interference cancellation and in-band jammer rejection," in 2018 IEEE Custom Integrated Circuits Conference (CICC). IEEE, 2018, pp. 1-4.

[30] D. Yang, H. Yüksel, and A. Molnar, "A wideband highly integrated and widely tunable transceiver for in-band full-duplex communication," IEEE Journal of Solid-State Circuits, vol. 50, no. 5, pp. 1189-1202, 2015.

[31] J. Zhou, N. Reiskarimian, and H. Krishnaswamy, "9.8 receiver with integrated magnetic-free n-path-filter-based non-reciprocal circulator and baseband selfinterference cancellation for full-duplex wireless," in 2016 IEEE International SolidState Circuits Conference (ISSCC). IEEE, 2016, pp. 178-180.

[32] A. Sergey and L. Norwood, "Mathematical handbook for electrical engineers," Artech House Inc, p. 139, 2005. 